\title{
13 Comets, Titan and Mars: Astrobiology and Space Projects
}

Yves Bénilan and Hervé Cottin

\section{Introduction}

All the solar system bodies were formed about 4.6 billion years ago, when a molecular cloud collapsed. The Sun, the telluric planets, the giant planets as well as their satellites, and the small bodies (asteroids and comets), were born from this material, which was probably quite homogeneously mixed in the beginning. From then, the physicochemical evolution yielded life at least on the Earth and also maybe on Mars or more speculatively on Europa. Thanks to space exploration, we try to figure out what makes the Earth such a peculiar object that it is the only one known to harbor life, and therefore, the extent to which life may have arisen somewhere else in the universe. To date, two factors appear to be of prime importance: a source of organic molecules and liquid water, the only solvent in which advanced molecular complexity appears to be possible to achieve, a preliminary condition for life. Henceforth, space missions dealing with astrobiology questions are conceived to answer these questions:

- What is the origin and the distribution of the organic matter in the solar system?

- Does water exist or did water exist in the liquid form on other objects?

The goal of this chapter consists of a brief review of space exploration dealing with three bodies of great astrobiological interest in our solar system: comets, Titan and Mars. Each of them is located at a specific level of our understanding of the origin of life on Earth, and the possibility that life could arise somewhere else. Comets as exogenous sources for prebiotic molecules, Titan as a model of an atmosphere in which endogenous syntheses of such molecules are very efficient, and finally Mars, which was the Earths "twin-sister planet" more than three billions years ago, where it is now established that liquid water once flowed, and thus might have once harbored life.

Yves Bénilan and Hervé Cottin, Comets, Titan and Mars: Astrobiology and Space Projects. In: Muriel Gargaud et al. (Eds.), Lectures in Astrobiology, Vol. II, Adv. Astrobiol. Biogeophys., pp. 347-428 (2007) 


\subsection{An Astrobiological Look at the Solar System}

\subsubsection{The Origin of the Organic Matter}

\subsubsection{The Exogenous Track}

Figure 13.1 is a scheme of the evolution of matter from our natal molecular cloud, to the different objects of the solar system. It shows, for each of them, the critical parameters that one considers favorable or unfavorable for life to

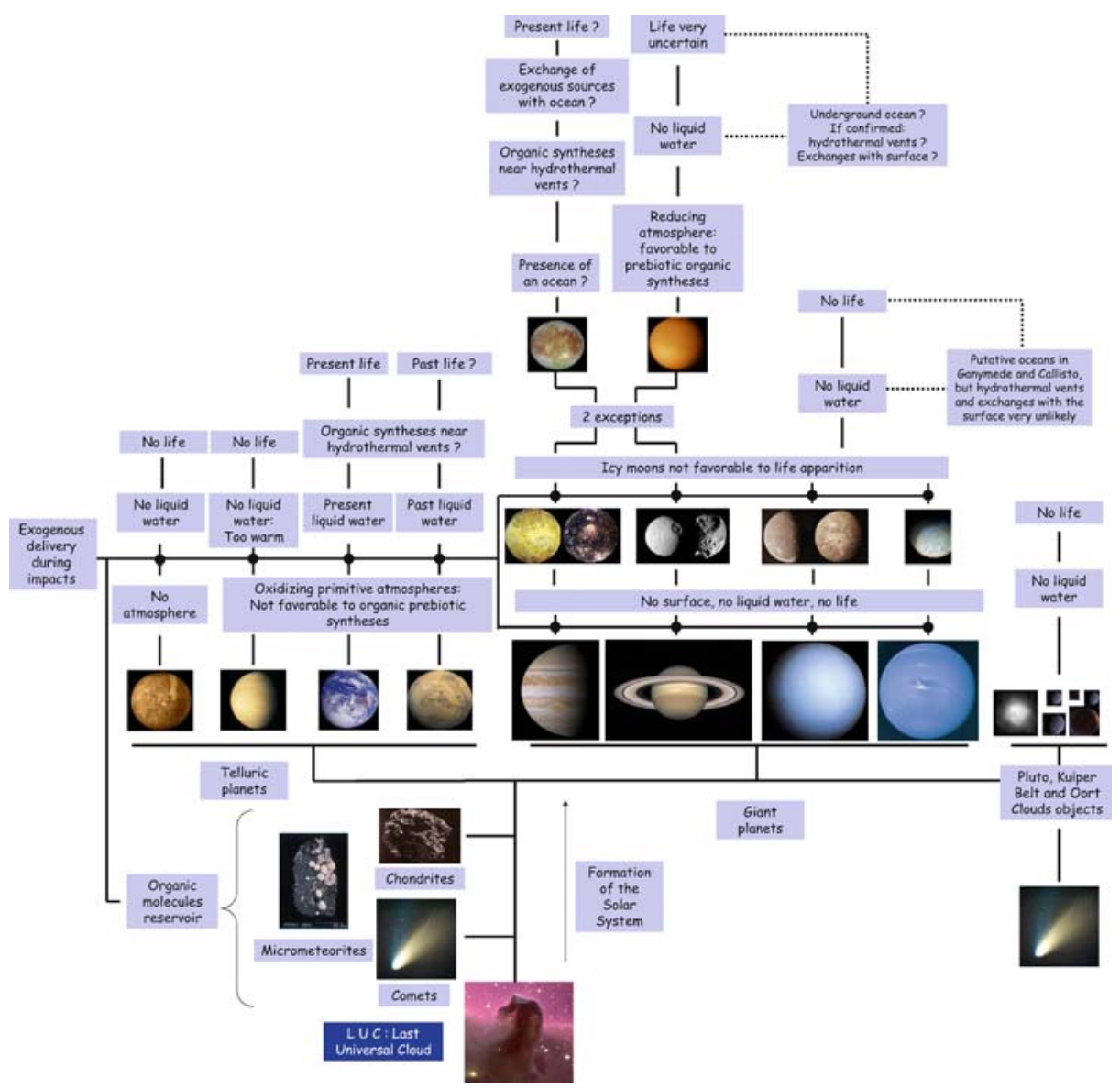

Fig. 13.1. Astrobiological tree of the solar system. The evolution of matter (see also Fig. 13.3) and astrobiological relevance of solar system objects. From bottom to top, and from left to right: the Horsehead nebula, comet C/1995 O1 (Hale-Bopp), Orgueil meteorite, Mercury, Venus, the Earth, Mars, Jupiter, Saturn, Uranus, Neptune, Pluto and objects of the Kuiper Belt and the Oort cloud, Io, Callisto, Mimas, Phoebe, Ariel, Oberon, Triton, Europa and Titan 
appear on those bodies. Everything starts with a molecular cloud (Fig. 13.1), which one might call LUC (last universal cloud) as an analogy with LUCA (last universal common ancestor) the common ancestor to all living organisms on the Earth, and LUCY who once was the oldest found human ancestor (see Chap. 11). Most certainly, the molecular clouds that we are now able to observe in our galaxy show strong analogies with the composition of our natal cloud LUC. Remote sensing observations have shown that organic chemistry is very active and advanced in such clouds, as well in the gaseous phase as in the solid phase (interstellar ices) (Ehrenfreund and Charnley 2000). $\mathrm{HCN}, \mathrm{HC}_{3} \mathrm{~N}$ or $\mathrm{HCHO}$ have been detected, and such molecules are of great astrobiological interest (Brack 2003; Oro and Cosmovici 1997). ${ }^{1}$ Moreover, a large number of experimental simulations in the laboratory predict that one might expect the existence of molecules much more complex than the ones already detected. Such experiments consist in simulating the chemistry occurring in interstellar ices, when submitted to irradiations (with photons or charged particles) or thermal cycles (Despois and Cottin 2005). In molecular clouds, a large amount of organic matter should be frozen on condensation nuclei made of silicates (Fig. 13.2). Some gravitational perturbation probably leads the cloud to collapse on itself, which resulted in the birth of our Sun and our planetary system.

A schematic view consists of considering that the original composition of interstellar grains was lost since they have been either incorporated in the Sun or planets, or pyrolysed in the vicinity of the Sun. Interstellar ices could also sublimate in the warmest parts of the nebula in which they have been brought by turbulent radial mixing in the solar nebula (Fig. 13.3). However, the extension of the turbulent region determines the possibility to keep pristine interstellar matter in comets. Inside the turbulent region, radial mixing brings interstellar

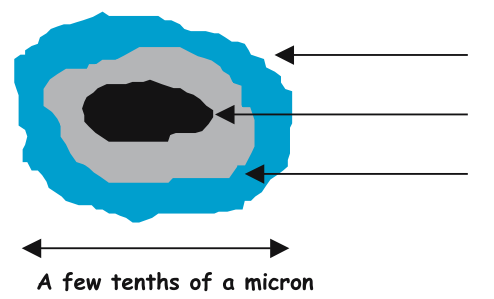

Ices of volatile compounds: $\mathrm{H}_{2} \mathrm{O}, \mathrm{CO}, \mathrm{CO}_{2}, \mathrm{NH}_{3}, \mathrm{CH}_{3} \mathrm{OH}, \mathrm{HCHO}$...

Silicate core

Mantle of refractory organic material

synthesised during processing of ices

\section{Dust Particle in a Molecular Cloud}

Fig. 13.2. Interstellar grain model. A silicate core on which volatile molecules condense in molecular clouds. The chemistry between those molecules leads to the synthesis of other compounds, with enhanced complexity. Our solar system formed with the accretion of such material. Some comets might have kept intact some of those grains

${ }^{1}$ In liquid water HCN chemistry leads to the synthesis of amino acids and puric bases (adenine and guanine), whereas $\mathrm{HC}_{3} \mathrm{~N}$ chemistry produces pyrimidic bases, and $\mathrm{HCHO}$ chemistry results in sugars or amino acids (when coupled with $\mathrm{HCN}$ ). 


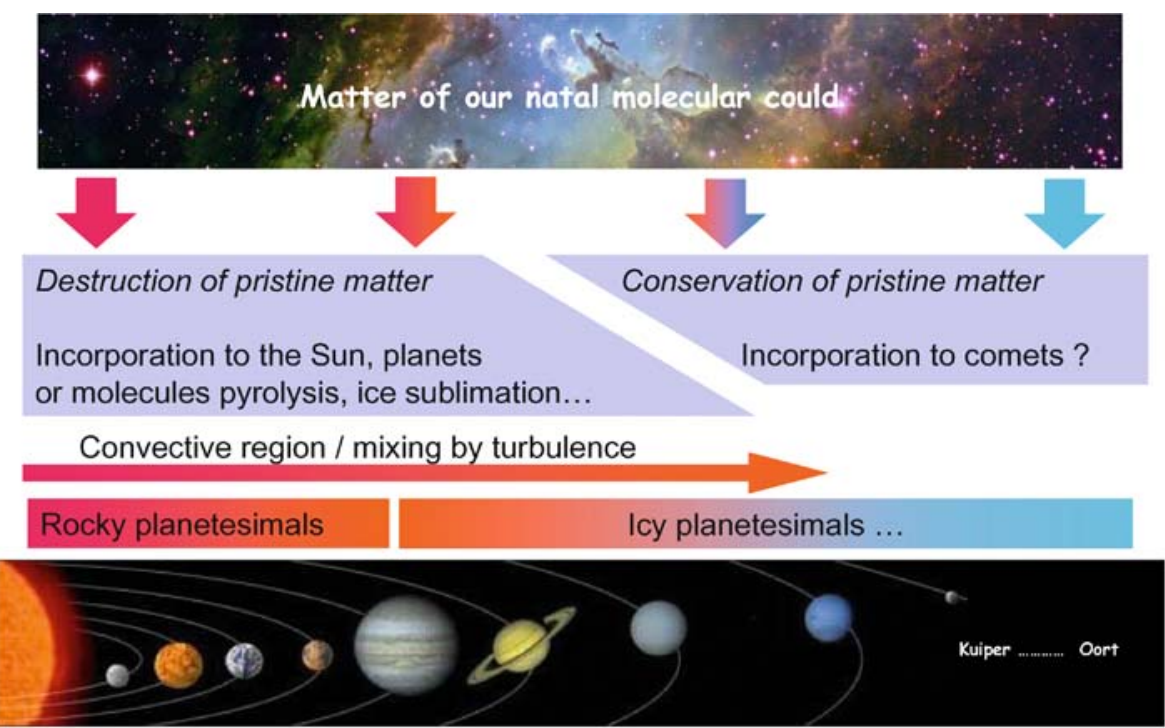

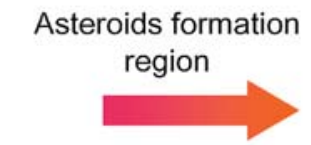

Pristine or differentiated
Comets formation

region

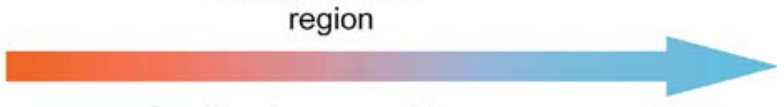

Gradient in composition

Fig. 13.3. Evolution of the matter between our natal molecular cloud, solar nebula and incorporation into the solar system bodies

grains close to the Sun, resulting in a loss of their initial composition. According to models, the turbulent region of the nebula can extend up to $30 \mathrm{AU}$ (BockeléeMorvan et al. 2002; Hersant et al. 2001). Beyond, grains might have remained in a cold environment, and thus have kept an unaltered interstellar composition. Several models result from those considerations, in which the interstellar organic matter undergoes different levels of transformation before it is stored in comets. They are sometimes considered as simple unaltered interstellar grain aggregates (Greenberg 1982), or from a rather different point of view, made of matter completely re-processed in the solar nebula (Prinn and Fegley 1989). Other models consider an intermediate scenario (Lunine et al. 1991; Iro et al. 2003), which are probably more realistic (see Despois and Cottin 2005 for more details on this topic). However, putting aside the discussion about the origin of the cometary matter, observations show an undeniable abundance of a large variety of organic compounds. Molecules such as $\mathrm{HCN}, \mathrm{HCHO}$ or $\mathrm{HC}_{3} \mathrm{~N}$, which have been detected in several comets, can have an origin in the interstellar medium or inside the nebula but keep the same astrobiological interest. Moreover, in situ measurements in the close vicinity of comets $1 \mathrm{P} /$ Halley (in 1986) and 81P/Wild 2 (in 2004) bear witness to the existence of more complex structures. These later re- 
main in the solid phase on dust grains when ejected from the nucleus, due to their high molecular mass (Huebner 1987; Kissel and Krueger 1987; Kissel et al. 2004; Mitchell et al. 1992). The lack of liquid water flowing in the nuclei over a long period of time does not allow to seriously consider the possibility of life on comets (even if short events of ice melting are possible for brief periods after the nucleus formation due to the radioactive decay of radioelements such as ${ }^{26} \mathrm{Al}$ (Podolak and Prialnik 1997)). Still, all detected molecules, to which the complex molecules inferred from laboratory experimental simulations can be added, turn comets into objects with great astrobiological potential as endogenous sources for prebiotic molecules. Comets are the target of several past, current and future space missions, on which we will focus later in this chapter.

Nevertheless, molecules of astrobiological interest can be destroyed by the same processes that lead to their formation once synthesized in the interstellar medium or in the solar nebula (UV, charged particles or thermal cycles). Experiments conducted in space, onboard the MIR station, or space capsules FOTON, have shown that amino acids can survive exposition to solar UVs if they are embedded in a mineral matrix (on cometary grains for example, but also in meteorites) (Barbier et al. 1998; Barbier et al. 2002; Boillot et al. 2002). After surviving a long stay in space, those molecules have to endure the energy release when comets and meteorites impact the Earth. Theoretical (Chyba et al. 1990) and laboratory (Blank et al. 2001) works have shown that amino acids can survive such collisions.

Meteorites and more specifically those belonging to the carbonaceous chondrite family are another exogenous source for organic molecules. Unlike comets, for which no direct analysis of the nucleus composition has ever been made, a large number of meteorites have been studied with the most sensitive instruments in laboratories on Earth since by definition meteorites are bodies reaching Earth surface (the most famous among them being Murchison, Murray and Orgueil). The current flux of meteorites is estimated to be about 10 tons/year (Bland et al. 1996), and was probably higher on the primitive Earth. Great amounts of organic molecules have been detected in meteorites: hydrocarbons, alcohols, carboxylic acids, amines, amides, heterocycles including uracil, adenine and guanine (Stoks and Schwartz 1979; Stoks and Schwartz 1981), more than 70 amino acids for the Murchison meteorite, and recently diamino acids in the same object (Meierhenrich et al. 2004). Those detections show obviously that molecules once synthesized in space are able to survive impacts. Enantiomeric excess at the level of a few percents have been measured for some amino acids in the Murchison and Murray meteorites (Cronin and Pizzarello 1997; Pizzarello and Cronin 2000). This could give us a key to understanding the origin of homochirality in living organisms on Earth. Unlike comets, it is clear that parent bodies of meteorites have gone through events with liquid water, which might have lead to more advanced stages of chemical evolution. No space mission has been currently developed to analyze the composition of carbonaceous asteroids, which are probably the parent bodies of chondrites. Therefore, it is not in the 
frame of this chapter to discuss further those objects. Our point is simply to underline the astrobiological importance of meteorites as an exogenous source of complex organic material. A very detailed review dealing with organic molecules in meteorites can be found in Botta and Bada (2002). However, a project to explore a carbonaceous asteroid could certainly be selected in the years to come, and this will give us important information about the evolution stage of those bodies and the origin of their organic component.

Micrometeorites are another vector for exogenous delivery of organic molecules. With an asteroidal or cometary origin, their current flux is estimated to be about 10000 tons a year. They slowly sediment in the terrestrial atmosphere and thus undergo little warming that could destroy their organic content. Amino acids have been detected in micrometeorites collected in Antarctica (Maurette 1998). Therefore, they could also have played an important role in the origin of life process.

These three different kinds of exogenous delivery methods (comets, meteorites and micrometeorites) not only occurred on Earth, but also throughout the solar system (Fig. 13.1). However, those ingredients require liquid water to reach an increased level of complexity that could have lead to life.

\subsubsection{The Endogenous Track}

During the 1950s, for the first time, Stanley Miller implemented an experiment that consisted of simulating the chemistry coupling of a model of the primitive Earth atmosphere and the oceans. He submitted a gaseous mixture made of $\mathrm{H}_{2}$, $\mathrm{CH}_{4}, \mathrm{NH}_{3}$ and $\mathrm{H}_{2} \mathrm{O}$ to an electric discharge that simulated lightning storms, this mixture being connected with a warm liquid water bulb. This experiment resulted in the detection of a large amount of organic molecules, including several amino acids (Miller 1953). Those measurements are the basis of the chemical evolution theory, showing that the chemistry between simple and abundant molecules (in planetary atmosphere in the case of Miller's experiment) synthesizes key compounds leading to the formation of life, as we know it on Earth. The choice of a reduced atmosphere $\left(\mathrm{C}\right.$ as $\mathrm{CH}_{4}$ and $\mathrm{N}$ as $\left.\mathrm{NH}_{3}\right)$ was motivated by the observations of the giant planets (detection of $\mathrm{H}_{2}, \mathrm{CH}_{4}$ and $\mathrm{NH}_{3}$ ), which are supposedly not to have evolved since their formation, and were therefore considered by Miller as good models for the primitive atmosphere of the telluric planets. Nevertheless, it is now considered that Earth's primitive atmosphere was dominated by $\mathrm{CO}_{2}$ and $\mathrm{N}_{2}$, just like Venus and Mars. Such a composition does not allow syntheses of complex organic molecules, as shown in Table 13.1. However, if the Earth mantle was less oxidized 3.8 billions years ago than today, an important amount of methane could have been emitted through volcanism (Kasting 1993; Selsis and Parisot 2001). In this case, endogenous "Miller kind syntheses" were possible. However, to date, we do not have any indication of the amount of reduced gas in Earth's primitive atmosphere. Unlike exogenous deliveries that are still observed nowadays, there is no evidence that atmospheric endogenous syntheses actually occurred in the Earth's prebiotic environment. 
Table 13.1. Organic molecules synthesized during "Miller-like" experiments as a function of the composition of the starting mixture (adapted from Raulin 2001). Compounds measured in the solid phase residues are usually detected after an acid hydrolysis

\begin{tabular}{|c|c|c|c|}
\hline \multirow{2}{*}{$\begin{array}{l}\text { Gaseous } \\
\text { mixture }\end{array}$} & \multirow{2}{*}{$\begin{array}{l}\text { Related } \\
\text { planetary } \\
\text { atmosphere }\end{array}$} & \multicolumn{2}{|c|}{ Organic products } \\
\hline & & Electric discharge & Photolysis \\
\hline $\begin{array}{l}\mathrm{CH}_{4}+\mathrm{NH}_{3} \\
+\mathrm{H}_{2} \mathrm{O}\left(+\mathrm{H}_{2}\right)\end{array}$ & Giant planets & $\begin{array}{l}\mathrm{RH} \text { (saturated } \\
\& \text { unsaturated) } \\
\mathrm{HCN} \& \text { other } \\
\text { nitriles (saturated), } \\
\mathrm{RCO}_{2} \mathrm{H}, \mathrm{H}_{2} \mathrm{CO} \\
\text { other aldehydes } \\
\text { Ketones \& alcohols } \\
\text { Solid: Amino acids } \\
\& \text { nitrogenated } \\
\text { heterocycles after } \\
\text { hydrolysis }\end{array}$ & $\begin{array}{l}\mathrm{RH} \text { (mostly sat) } \\
\mathrm{HCN} \\
\mathrm{RCN} \text { (saturated) } \\
\text { if } \mathrm{N} / \mathrm{C}<1 \\
\mathrm{RNH}_{2} \text { if } \mathrm{N} / \mathrm{C}>1 \\
\mathrm{H}_{2} \mathrm{CO} \text {, other } \\
\text { aldehydes } \\
\text { Ketones \& alcohols } \\
\text { Solid: Amino acids } \\
\text { after hydrolysis }\end{array}$ \\
\hline $\begin{array}{l}\mathrm{CH}_{4}+\mathrm{N}_{2} \\
\left(+\mathrm{H}_{2} \mathrm{O}\right)\end{array}$ & Titan, Triton & $\begin{array}{l}\mathrm{RH} \text { (saturated } \\
\& \text { unsaturated) } \\
\mathrm{HCN} \text { \& other nitriles } \\
\text { (saturated \& unsatu- } \\
\text { rated) including } \mathrm{HC}_{3} \mathrm{~N} \text {, } \\
\text { other aldehydes } \\
\text { and } \mathrm{C}_{2} \mathrm{~N}_{2}, \mathrm{H}_{2} \mathrm{CO} \\
\text { Ketones \& alcohols } \\
\text { Solid: Amino acids } \\
\& \text { nitrogenated hetero- } \\
\text { cycles after hydrolysis }\end{array}$ & $\begin{array}{l}\mathrm{RH} \text { (saturated } \\
\& \text { unsaturated) } \\
\mathrm{H}_{2} \mathrm{CO} \& \text { other } \\
\text { aldehydes with } \\
\text { low yields }\end{array}$ \\
\hline $\begin{array}{l}\mathrm{CO}+\mathrm{NH}_{3} \\
+\mathrm{H}_{2} \mathrm{O}\end{array}$ & & $\begin{array}{l}\text { HCN, oxygenated organ } \\
\text { Solid: Amino acids after }\end{array}$ & $\begin{array}{l}\text { compounds } \\
\text { hydrolysis }\end{array}$ \\
\hline $\begin{array}{l}\mathrm{CO}_{2}+\mathrm{N}_{2} \\
+\mathrm{H}_{2} \mathrm{O} \\
+\mathrm{CO} / \mathrm{H}_{2}\end{array}$ & Primitive Earth? & $\begin{array}{l}\mathrm{RH} \text { (mostly saturated) } \\
\mathrm{HCN} \text {, other nitriles (sat } \\
\mathrm{H}_{2} \mathrm{CO} \text {, other aldehydes, } \\
\text { Solid: Amino acids after }\end{array}$ & $\begin{array}{l}\text { rated) } \\
\text { retones } \\
\text { hydrolysis }\end{array}$ \\
\hline $\begin{array}{l}\mathrm{CO}_{2}+\mathrm{N}_{2} \\
+\mathrm{H}_{2} \mathrm{O}\end{array}$ & $\begin{array}{l}\text { Primitive Earth? } \\
\text { Venus, Mars }\end{array}$ & No synthesis & \\
\hline
\end{tabular}


However, unlike the Earth, Titan, the largest moon of Saturn, has a noticeable fraction of methane in addition to an atmosphere mainly composed of nitrogen. As shown in Table 13.1, organic syntheses are very efficient in such an environment, which is confirmed by observations since we can observe a thick layer of organic aerosols resulting from $\mathrm{N}_{2} / \mathrm{CH}_{4}$ complex chemistry. Yet, the temperature of the satellite does not allow liquid water on the surface. Therefore, Titan can be considered as a laboratory at the planetary scale in which we can study the level of complexity that chemical evolution can reach without any water in the liquid phase. Clearly, there is no serious reason to consider that life might have appeared on Titan. Nevertheless, the presence of oceans is possible under a deep ice layer (Fortes 2000), but transport between surface organics and underground liquid water is still an open issue. Titan is the destination of the European probe Huygens, which will be later developed in this chapter.

Finally, another kind of endogenous source of organic compounds has to be considered: a synthesis at the bottom of the oceans, in hydrothermal vents (also known as black smokers) (Corliss et al. 1981). When oceanic plates are drifting apart, water infiltrates the crust and springs from black smokers at high temperatures, enriched in gas $\left(\mathrm{H}_{2}, \mathrm{~N}_{2}, \mathrm{CH}_{4}, \mathrm{H}_{2} \mathrm{~S}\right.$, etc. $)$ and minerals. It has been shown experimentally that amino acids can be synthesized in such conditions (high temperature and pressure, reduced environment, and minerals which can act as a catalyst) (Hennet et al. 1992; Yanagawa and Kobayashi 1992). However, such molecules are also very efficiently destroyed due to the very high temperatures in those environments. More experimental data and field measurements are required to assess the feasibility of this mechanism.

\subsubsection{Follow the Water}

Water in the liquid state seems to be the most favorable solvent to allow molecules with an exogenous or endogenous origin to reach more evolved structures, and thus to make possible the appearance of life. If the $\mathrm{H}_{2} \mathrm{O}$ molecule is ubiquitous in the solar system and the galaxy, it is most of the time in the gaseous phase (Venus, Mars, giant planets, interstellar medium) or the solid phase (giant planets icy moons, comets, interstellar ices). To date, the presence of water in the liquid state has only been established on the Earth. Yet, two solar system objects are of prime interest: Mars and Europa.

\subsubsection{Mars}

NASA's current strategy for Martian exploration is summarized in the expression: "follow the water". Even if nowadays there is no evidence of liquid water on the surface of the red planet, the record of past aqueous flows can be read in the landscapes morphology, and in the composition of some minerals or rocks, which can only be formed in the presence of liquid water. A large number of space missions to Mars slowly lift the veil on its past. As we will see later in 
this chapter (see also Chap. 4), it is now quite well-established that an abundant amount of water once flowed on Mars (rivers, or even an ocean covering almost the whole northern hemisphere), and this, at a time period during which life had possibly already appeared on Earth. Thus, a source of organic molecules (the Martian primitive atmosphere being probably similar to the Earth's, the exogenous source hypothesis is the most likely) combined with liquid water, could have induced the appearance of life just like on Earth. However, attempts to detect organic molecules on Mars have not yet been successful. Today, those molecules and possible liquid water pockets, if any, are probably buried underground, and future space missions will have to dig the oxidized soil of the planet to add a new dimension to our knowledge of Mars. If life developed on Mars, independently from the Earth ${ }^{2}$, this could mean that the jump from chemistry to biology is written in the laws of the natural evolution of organic matter each time the requirements "organic matter plus liquid water" are fulfilled.

\subsubsection{Europa and the Icy Moons of the Giant Planets}

Observations of the Jovian satellite Europa from the space probe Galileo lead to an accumulation of data that converge to the hypothesis that an ocean is buried under its icy surface (morphological clues (Carr et al. 1998), detection of a magnetic field (Kivelson et al. 2000), and hydrated salts at the surface (McCord et al. 1999)). The ocean could have been created by the heating of the planet due to Jupiter's gravitational field, which puts Europa out of shape and induces important stresses on the ice. It could be hundreds of kilometers deep and would be covered with an icy layer of thickness 10 to $50 \mathrm{~km}$ (Fig. 13.4) (Sotin et al. 2002), which casts the feasibility of a submarine exploration mission into the rather distant future. Nevertheless, external exploration missions are currently considered to confirm the presence of an ocean under the ices of Europa: JIMO (Jupiter Icy Moons Orbiter) was an example of project for such a mission. ${ }^{3}$

However, the association between liquid water and a source of organic molecules is still problematic on Europa. According to the thickness of the ice layer, exchanges between exogenous deliveries at the surface and the ocean are possible but could be very limited (Pierazzo and Chyba 2002; Schenk 2002). Concerning endogenous sources, in the absence of an atmosphere, organic syntheses in the vicinity of black smokers could be the only way to synthesize prebiotic compounds. Indeed, theoretical models have shown that the putative ocean of Europa could be in contact with the silicate mantle of the planet. If an inner

\footnotetext{
${ }^{2}$ It is also possible that life appeared on only one of the two planets, and then was exported to the other: Martian meteorites have indeed been collected on Earth showing that such a travel is feasible, one of these meteorites contains very controversial hints of past microbial activity: ALH 84001. Experiments implemented in space have shown that some microorganisms can survive such an interplanetary journey.

${ }^{3}$ For more information about JIMO: http://en.wikipedia.org/wiki/Jupiter_Icy_Moons_Orbiter
} 


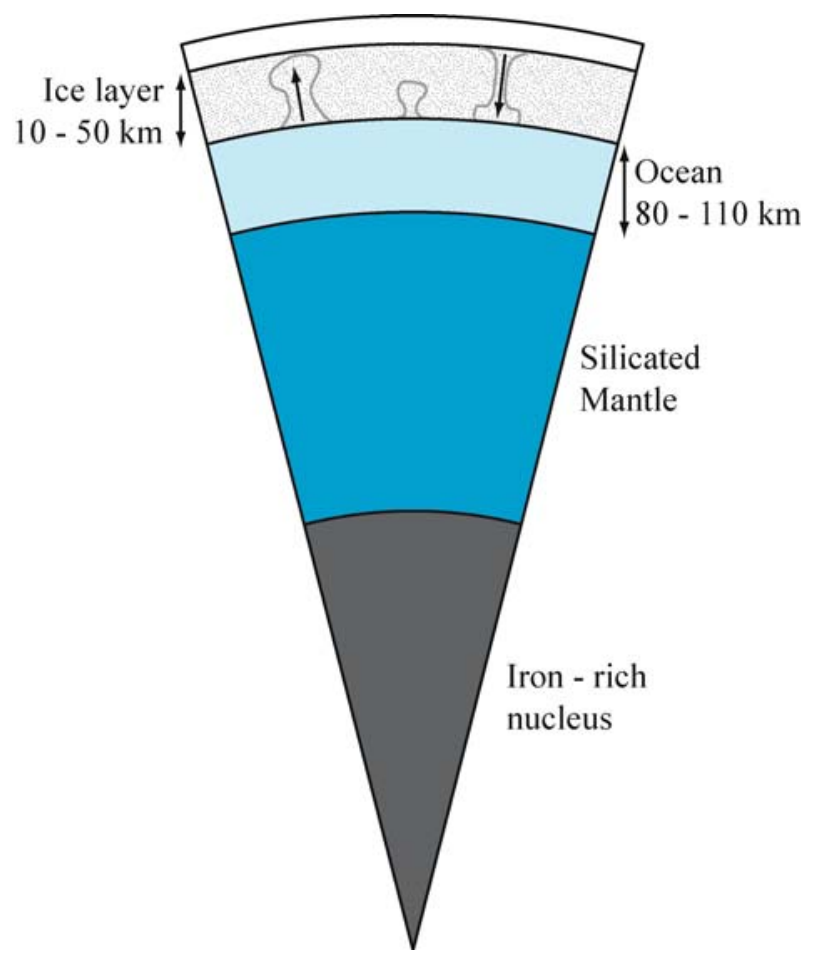

Fig. 13.4. A representation of the internal structure of Europa (Sotin et al. 2002)

source of heat from inside the satellite does exist, hydrothermal vents could be present at the bottom of the ocean, even if these considerations are still very speculative. Thus, a conjunction of those favorable conditions could make Europa another object of the solar system currently harboring life.

The presence of oceans is also considered inside Ganymede (McCord et al. 2001) and Callisto (Zimmer et al. 2000). However, in both cases, they would be embedded at larger depths than on Europa, and trapped between two ice layers, where the presence of hydrothermal vents is not possible. Ganymede and Callisto are also targets of the JIMO mission.

\subsection{The Space Exploration of Comets}

\subsubsection{General Considerations}

As already mentioned in this chapter, comets, as are planets, are made of matter from a molecular cloud, which collapsed to give birth to our solar system. Those icy planetesimals have not been through differentiation processes thanks to their small size; they have been stored in the outermost, hence coldest, regions of the 
solar system (Kuiper Belt and Oort Cloud). Therefore their initial composition should remain unchanged and thus bear testimony of the physical and chemical environment prevailing in the solar nebula region in which they have been formed. Some might even kept the same composition as our natal molecular cloud (Fig. 13.3). Therefore, comets are considered as the oldest archives of our planetary system, as if samples of pristine matter were stored in a freezer to be preserved. A gravitational perturbation or a collision can eject a cometary nucleus out of its reservoir and place it on an elliptical orbit that will lead it close to the Sun. This approach triggers the sublimation of the ices (first CO, then $\mathrm{H}_{2} \mathrm{O}$ and other volatile compounds which are frozen far from the Sun) and leads to the formation of the cometary atmosphere (the coma) and of the tails that can sometimes be seen with naked eyes from the Earth (Fig. 13.5) (much more about comets can be found in Despois and Cottin 2005).

Most of the currently available data about the composition of comets are derived from observations by teledetection methods from Earth. To date, about twenty molecules have been detected in the gaseous phase (mainly $\mathrm{H}_{2} \mathrm{O}$, but also $\mathrm{CO}, \mathrm{CO}_{2}, \mathrm{CH}_{3} \mathrm{OH}, \mathrm{NH}_{3}, \mathrm{HCN}, \mathrm{CH}_{4}$, etc.), but there is no direct information about the molecular composition of cometary nuclei (Bockelée-Morvan et al. in press; Despois and Cottin 2005). Those observations are the starting point for a large number of laboratory experiments, which consist of simulating the behavior of ices made of molecules detected in the coma. Those ices are

Ion tail (up to 100 millions $\mathrm{km}$ )

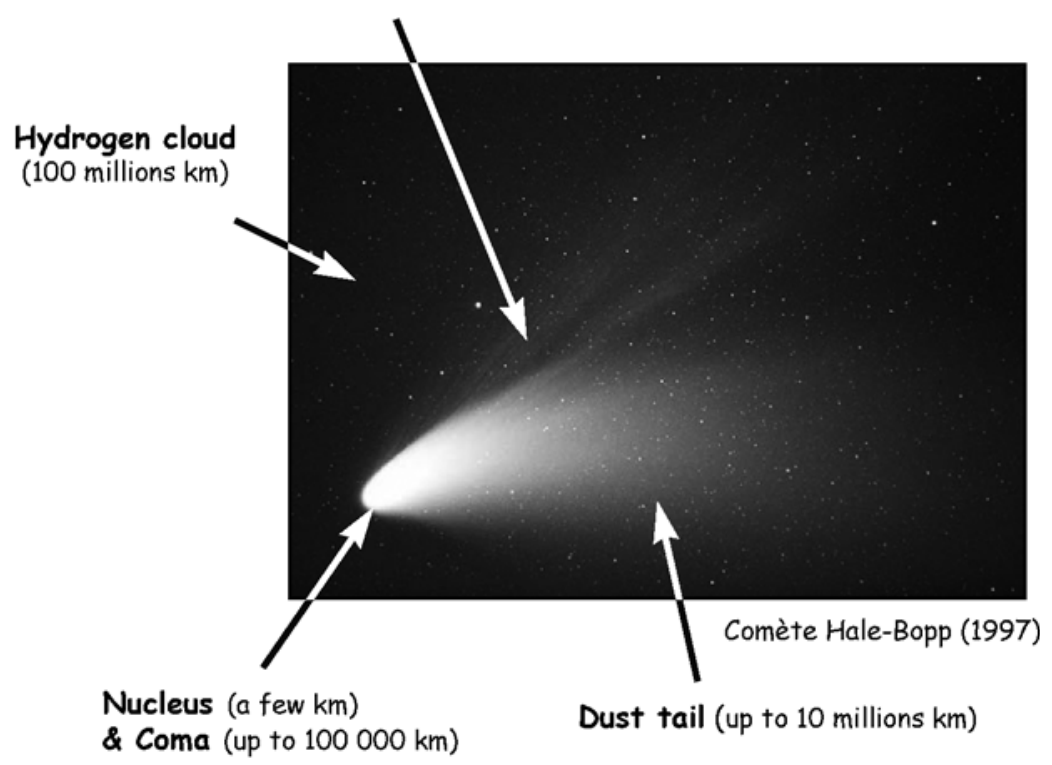

Fig. 13.5. Structure of a comet at about $1 \mathrm{AU}$ from the Sun 
submitted to different kinds of energy sources (UV photons, charged particles, thermal cycles) simulating the conditions they encountered during their history (where history can go back to long before being incorporated into comets, maybe even into the interstellar medium). Those experiments yield the production of molecules much more complex than the starting material, and are a sign for great complexity of the nucleus in terms of organic composition (Table 13.2).

Table 13.2. Molecules detected during experimental simulations of cometary and interstellar ice analogues. Italic letters refer to molecules actually detected in comets, $(t)$ means tentative detection only in the analogues. Amino acids (alanine, AIB, and so on, except glycine) were detected after acid hydrolysis of the room temperature residue (from Despois and Cottin 2005)

Hydrocarbons:
$\mathrm{CH}_{4}$
$\mathrm{C}_{2} \mathrm{H}_{2}, \mathrm{C}_{2} \mathrm{H}_{4}, \mathrm{C}_{2} \mathrm{H}_{6}$
$\mathrm{C}_{3} \mathrm{H}_{8}, \mathrm{C}_{4} \mathrm{H}_{10}$
$\mathrm{C}_{5} \mathrm{H}_{10}, \mathrm{C}_{5} \mathrm{H}_{12}$
$\mathrm{C}_{6} \mathrm{H}_{12}, \mathrm{C}_{6} \mathrm{H}_{14}$
$\mathrm{C}_{7} \mathrm{H}_{16}$

Amides:

$\mathrm{NH}_{2} \mathrm{CHO}$

$\mathrm{CH}_{3} \mathrm{CONH}_{2}$ $\mathrm{HOCH}_{2} \mathrm{CONH}_{2}$ $\mathrm{NH}_{2}(\mathrm{CO})_{2} \mathrm{NH}_{2}$ $\mathrm{HOCH}_{2} \mathrm{CH}(\mathrm{OH}) \mathrm{CONH}_{2}$

\section{Amines: \\ $\mathrm{HOCH}_{2} \mathrm{CH}_{2} \mathrm{NH}_{2}$ $\mathrm{HCNH}\left(\mathrm{NH}_{2}\right)$ Diaminopyrrole Diaminofurane Triaminopropane $\left(\mathrm{CH}_{2}\right)_{6} \mathrm{~N}_{4}(\mathrm{HMT})$}

Aldehydes:

$\mathrm{H}_{2} \mathrm{CO}$ $\mathrm{CH}_{3} \mathrm{OCH}_{2} \mathrm{CHO}(\mathrm{t})$

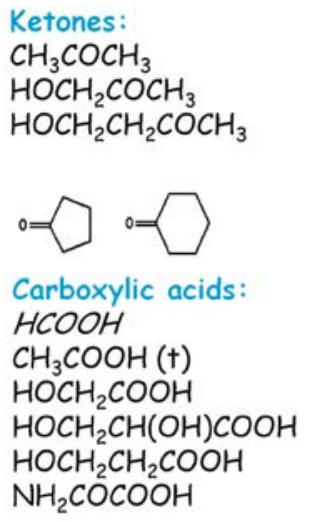

Aromatic Compounds:

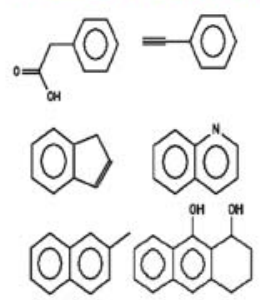

Ethers: $\mathrm{CH}_{3} \mathrm{OCH}_{2} \mathrm{OCH}_{3}(t)$ $\mathrm{C}_{3} \mathrm{H}_{6} \mathrm{O}_{3}$ (Trioxane) $(t)$ $\left(-\mathrm{CH}_{2}-\mathrm{O}-\right)_{n}(\mathrm{POM})$
Alcohols:

$\mathrm{CH}_{3} \mathrm{OH}$

$\mathrm{CH}_{3} \mathrm{CH}_{2} \mathrm{OH}$

$\mathrm{HOCH} \mathrm{CH}_{2} \mathrm{OH}$

$\mathrm{HOCH}_{2} \mathrm{CH}(\mathrm{OH}) \mathrm{CH}_{2} \mathrm{OH}$

$\mathrm{C}_{4} \mathrm{H}_{8}(\mathrm{OH})_{2}$

$\mathrm{C}_{5} \mathrm{H}_{9} \mathrm{OH}(\dagger)$

$\mathrm{C}_{5} \mathrm{H}_{11} \mathrm{OH}$

Amino Acids:

$\mathrm{NH}_{2} \mathrm{CH}_{2} \mathrm{COOH}$ (Glycine)

$\mathrm{NH}_{2} \mathrm{CH}\left(\mathrm{CH}_{3}\right) \mathrm{COOH}$ (Alanine)

$\mathrm{CH}_{3} \mathrm{CH}_{2} \mathrm{CH}\left(\mathrm{NH}_{2}\right) \mathrm{COOH}(\alpha A B A)$

$\mathrm{CH}_{3} \mathrm{CH}\left(\mathrm{NH}_{2}\right) \mathrm{CH}_{2} \mathrm{COOH}(\mathrm{ABA})$

$\left(\mathrm{CH}_{2} \mathrm{NH}_{2}\right)\left(\mathrm{CH}_{3}\right) \mathrm{CHCOOH}$ (AIBA)

Sarcosine

Ethylglycine

Valine, Proline, Serine

Aspartic acid

Diaminopropanoic acid

Diaminobutyric acid

Diaminopentanoic acid

Diaminohexanoic acid
Esters:

$\mathrm{HCOOCH}_{3}$ $\mathrm{CH}_{3} \mathrm{COOCH}_{3}$ $\mathrm{CH}_{3} \mathrm{CH}_{2} \mathrm{COOCH}_{3}$

Others: $\mathrm{CO}, \mathrm{CO}_{2}, \mathrm{C}_{3} \mathrm{O}_{2}, \mathrm{H}_{2} \mathrm{O}_{2}, \mathrm{H}_{2} \mathrm{CO}_{3}, \mathrm{~N}_{2} \mathrm{H}_{4}, \mathrm{HNCO}, \mathrm{NH}_{2} \mathrm{CONH}_{2}$, $\mathrm{NH}_{2} \mathrm{CONHCONH}{ }_{2}$ 
Space missions are a necessary complement to observation from the Earth and laboratory experimental simulations.

Finally, since the nucleus is hidden behind the coma, its observation from the Earth is impossible. If the comet is not active, the nucleus is much too far and too dark to be observed. Thus, we have the pictures of only four cometary nuclei, which have been taken from space probes (Fig. 13.6).

\subsubsection{Past Missions}

Past cometary space missions are presented in Table 13.3 and Fig. 13.7. It is not possible to summarize all the results obtained from those missions in the frame of this chapter; therefore, we have chosen to focus more specifically on astrobiology related data.

Organic compounds with molecular weights higher than those observed by teledetection methods (from the Earth or from space probe instruments) have

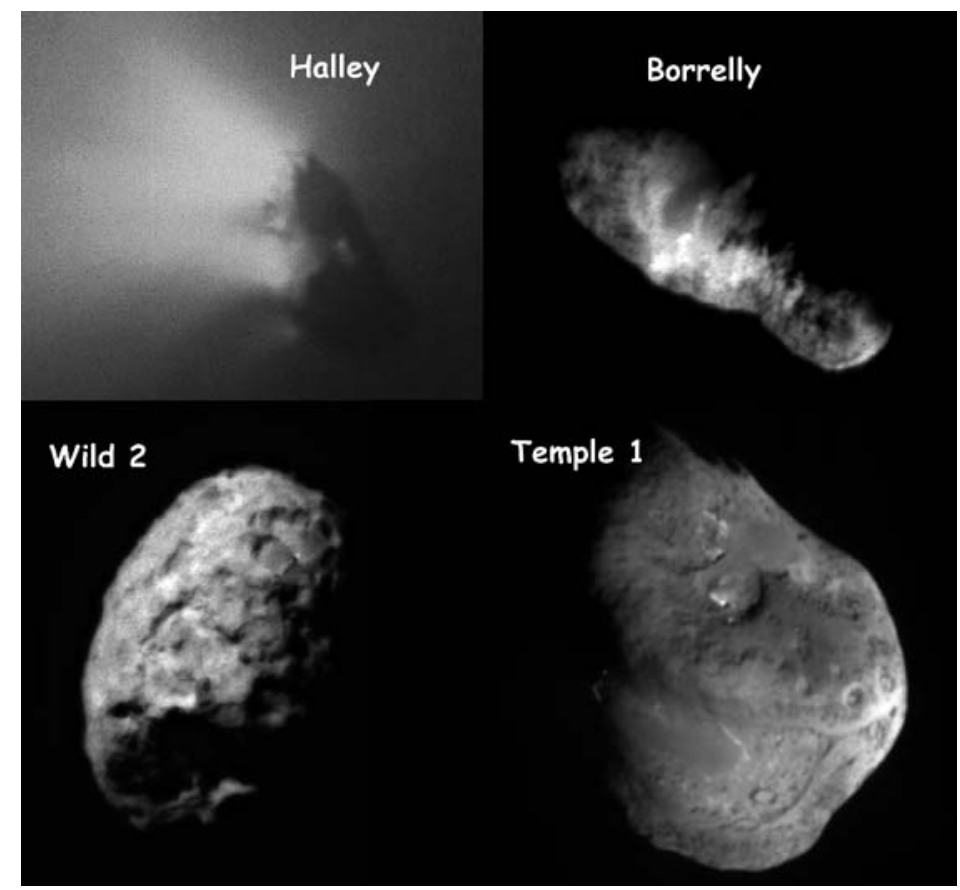

Fig. 13.6. The four cometary nuclei observed to date: Halley from the Giotto spacecraft in 1986 (distance at picture time: $\sim 700 \mathrm{~km}$, nucleus: $4.1 \times 4.2 \times 8 \mathrm{~km}$ ) (courtesy of ESA/MPIfA); Borrelly from the Deep Space 1 spacecraft in 2001 (distance: $3417 \mathrm{~km}$, nucleus: $3.2 \times 8 \mathrm{~km}$ ) (courtesy of NASA/JPL); Wild 2 from Stardust in 2004 (distance: $500 \mathrm{~km}$, nucleus: $\sim 5 \mathrm{~km}$ ) (courtesy of NASA/JPL); Temple 1 from Deep Impact in 2005 (distance: $3000 \mathrm{~km}$, nucleus: $\sim 5 \times 7 \mathrm{~km}$ ) (courtesy of NASA/JPL-Caltech/UMD) 


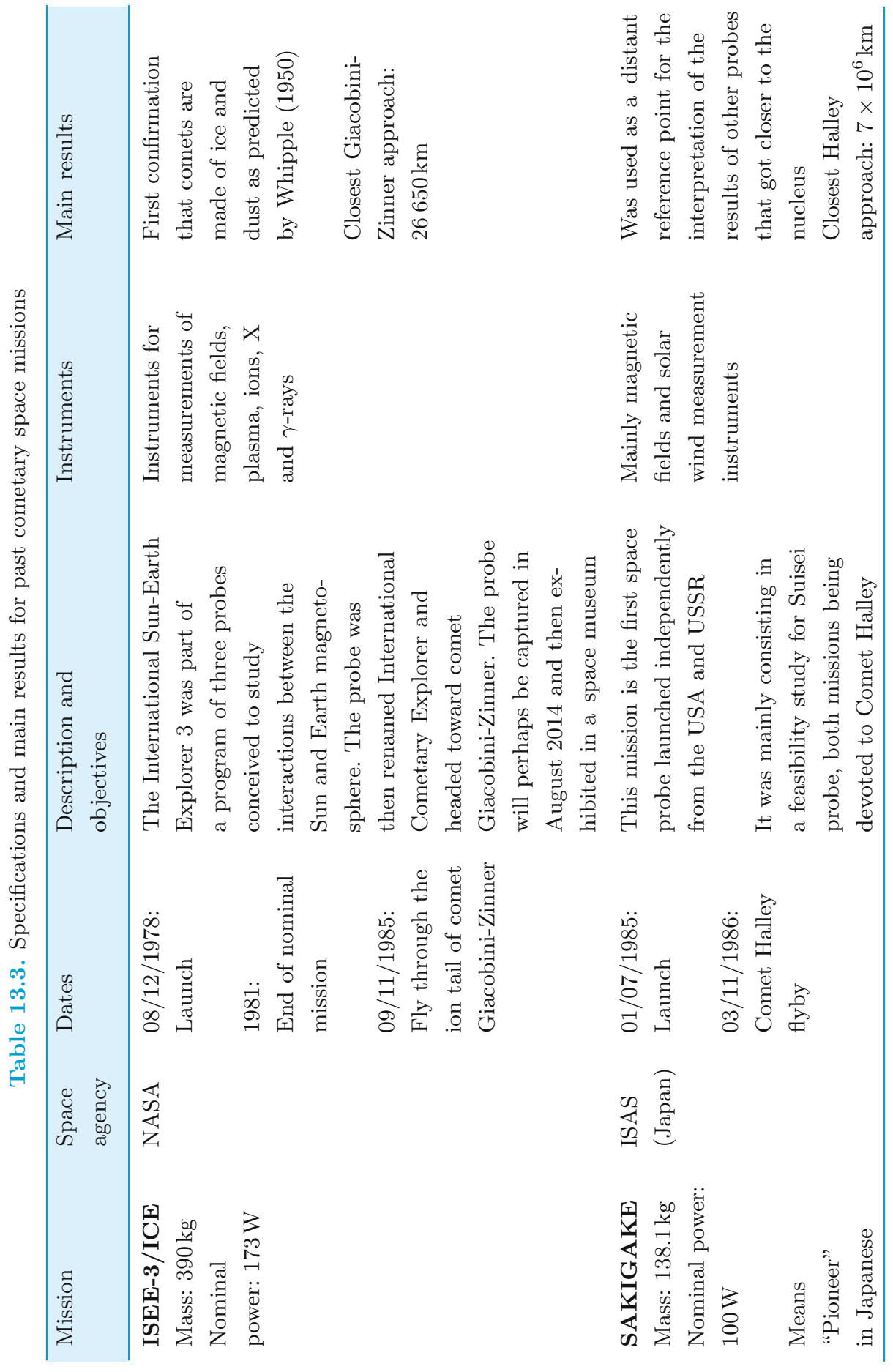




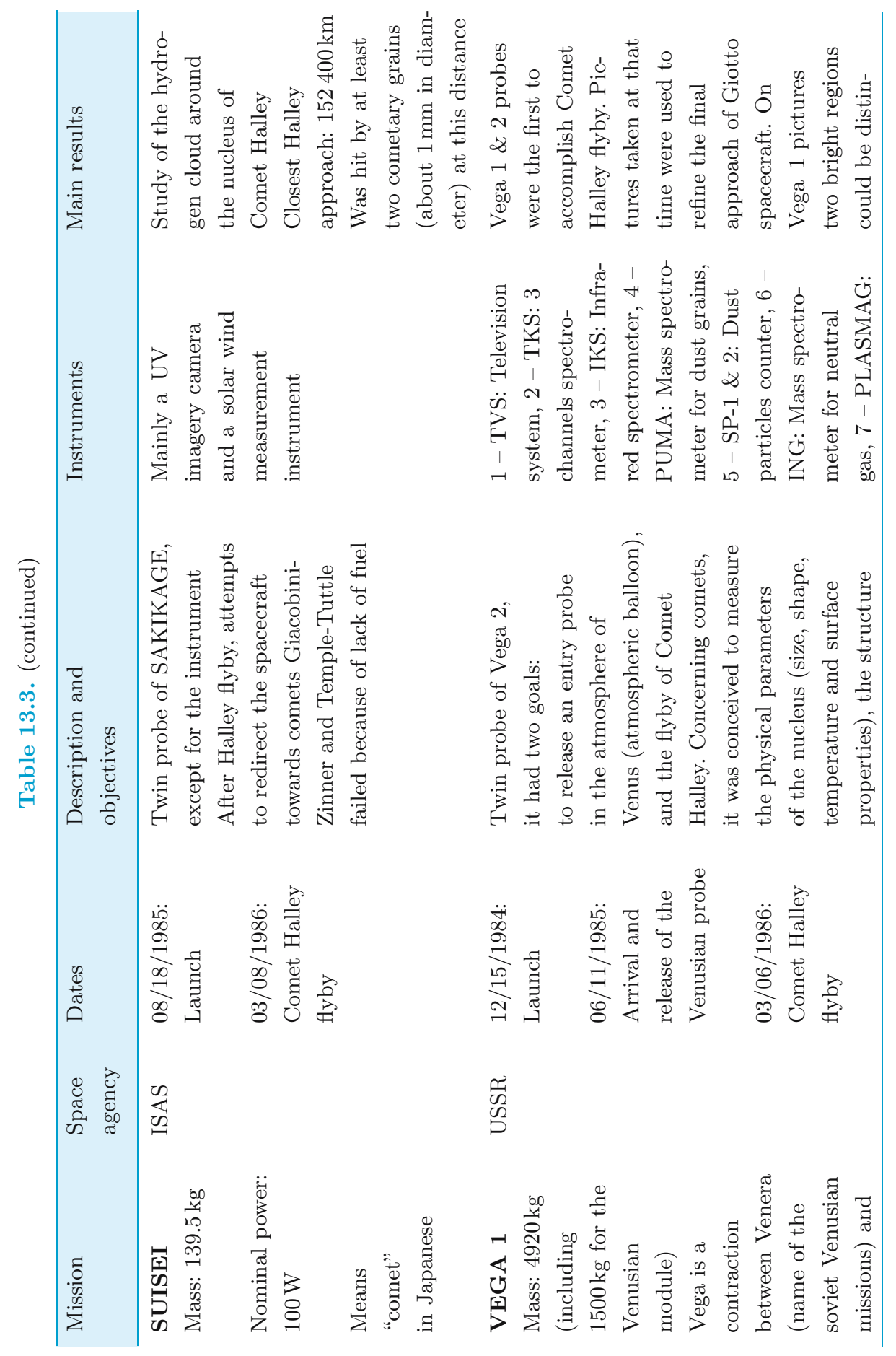




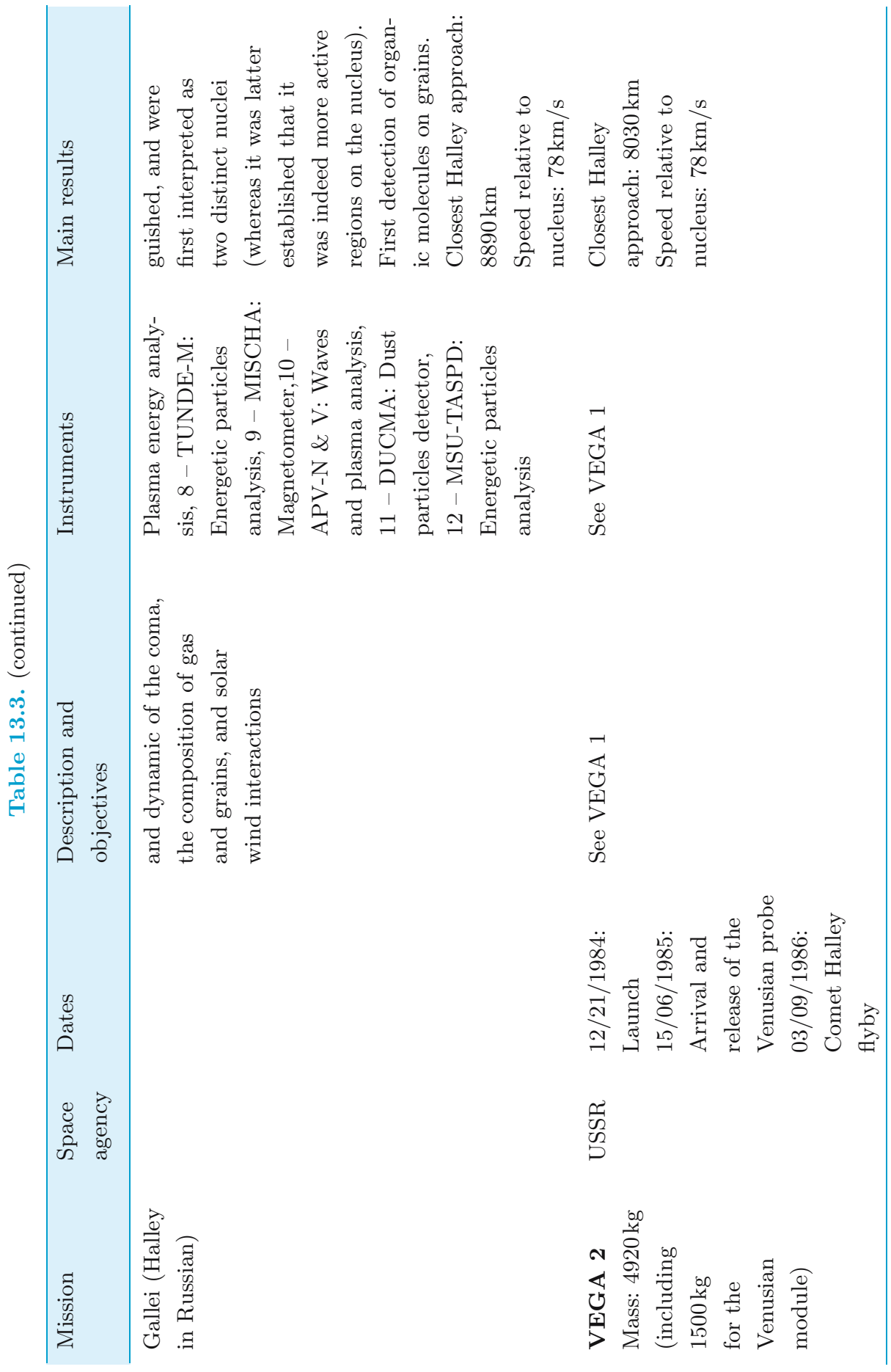




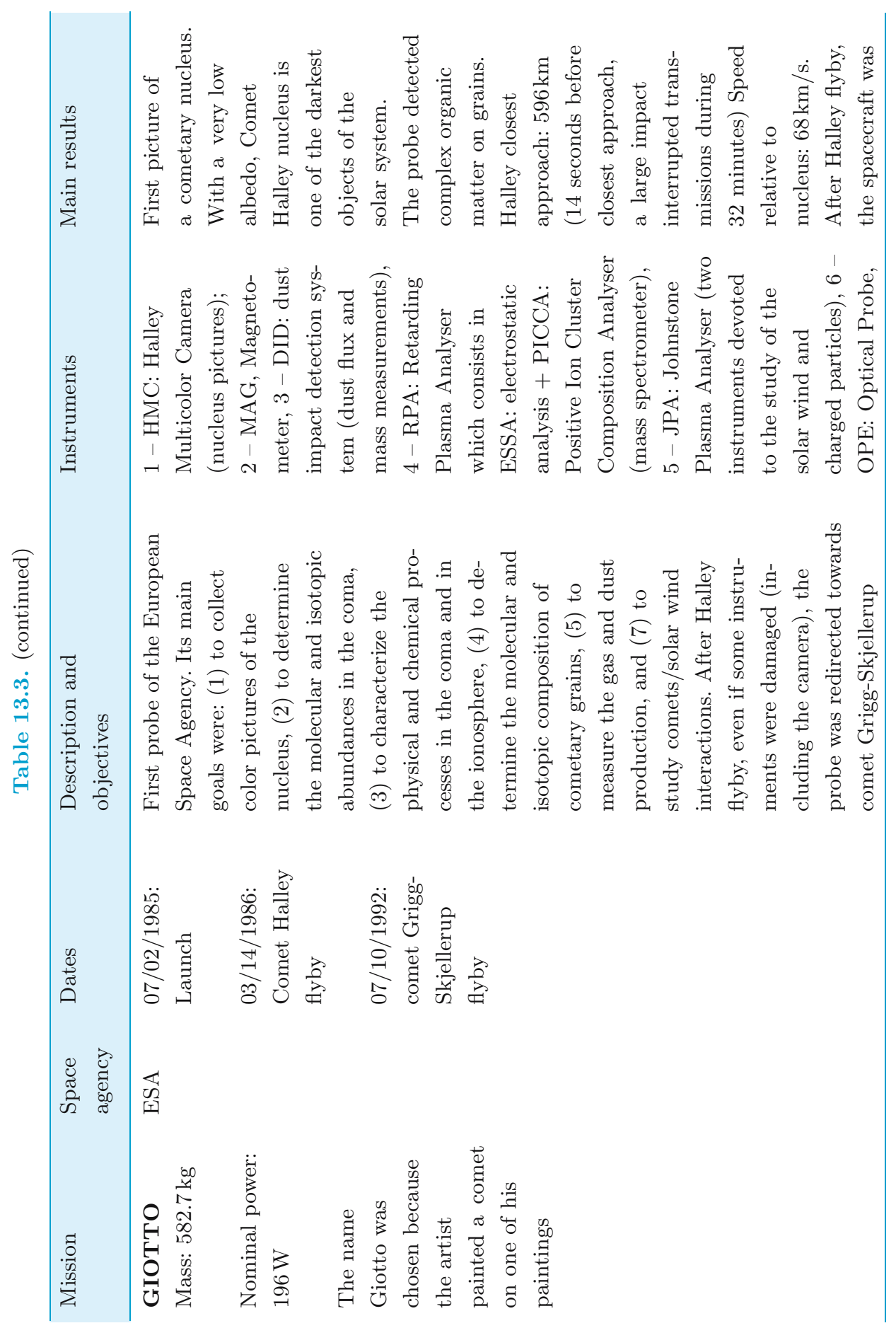




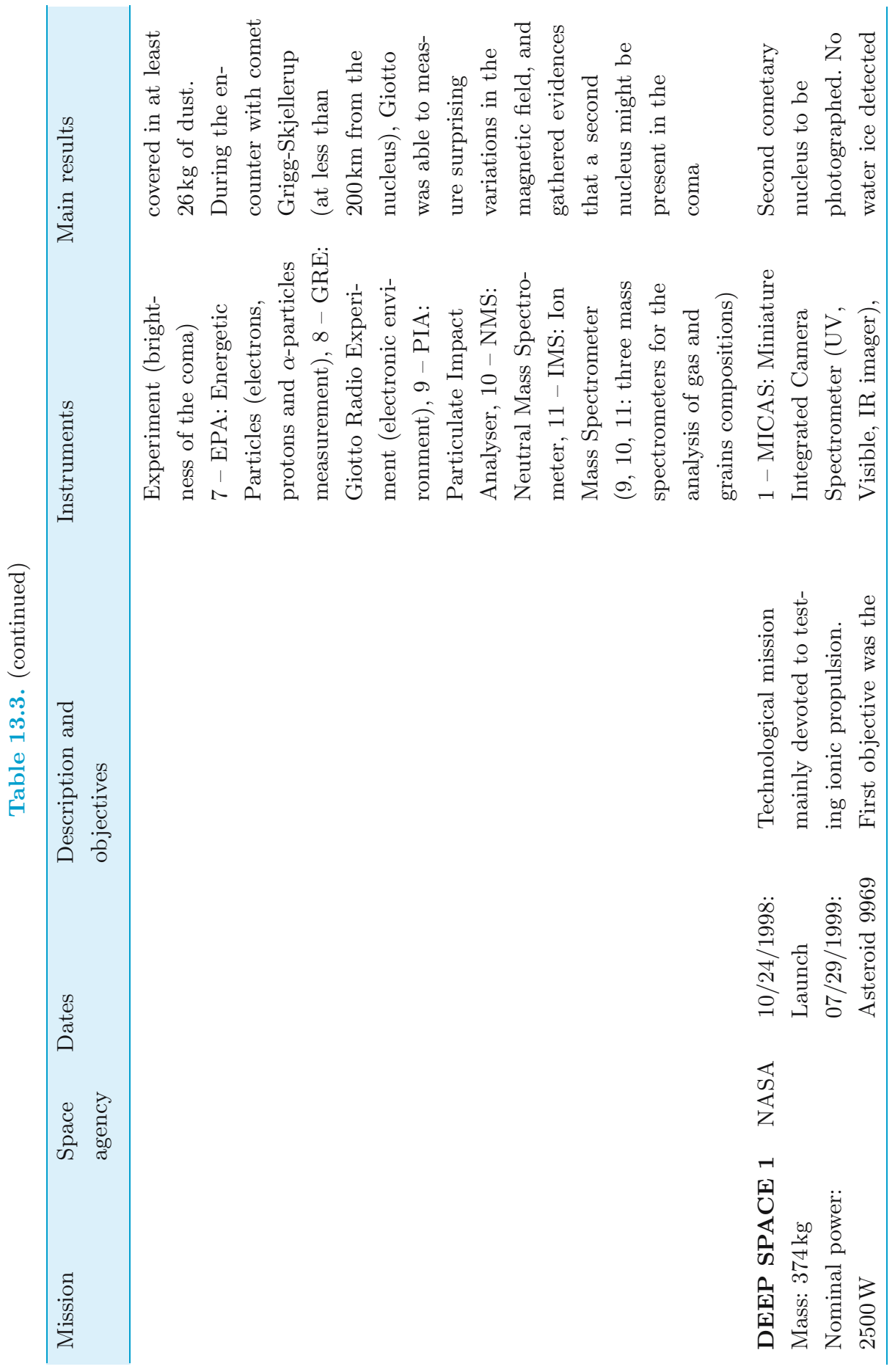




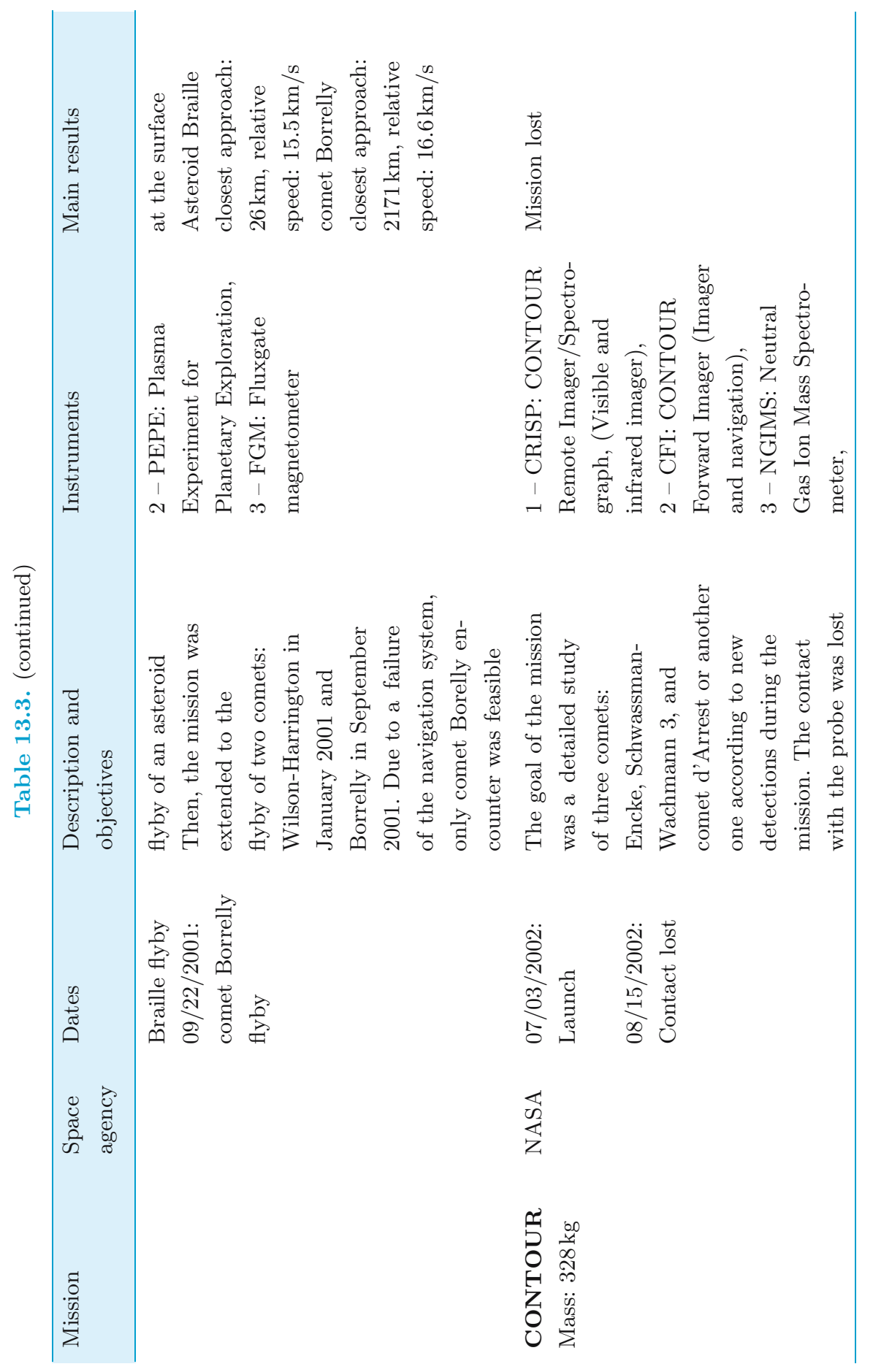




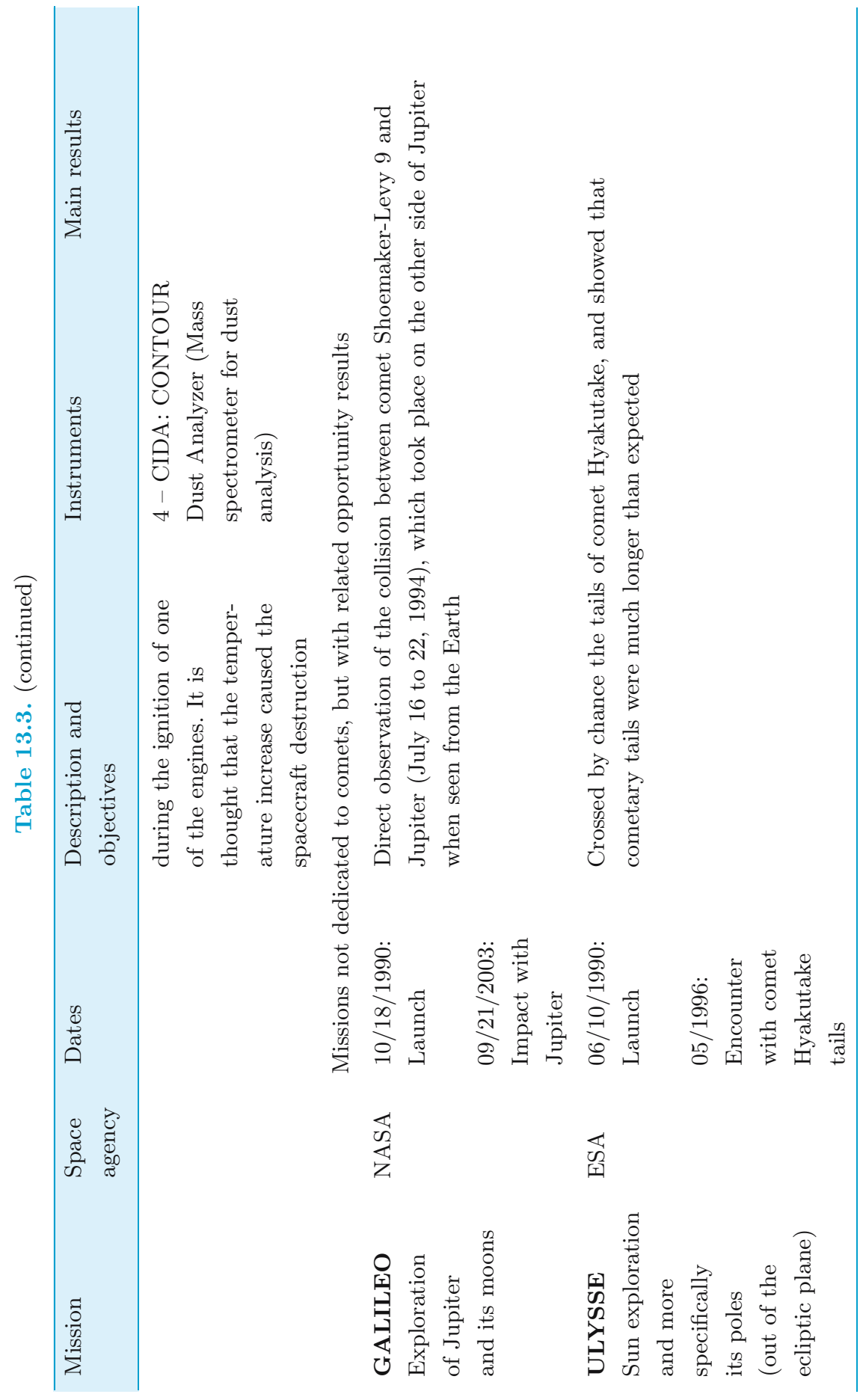




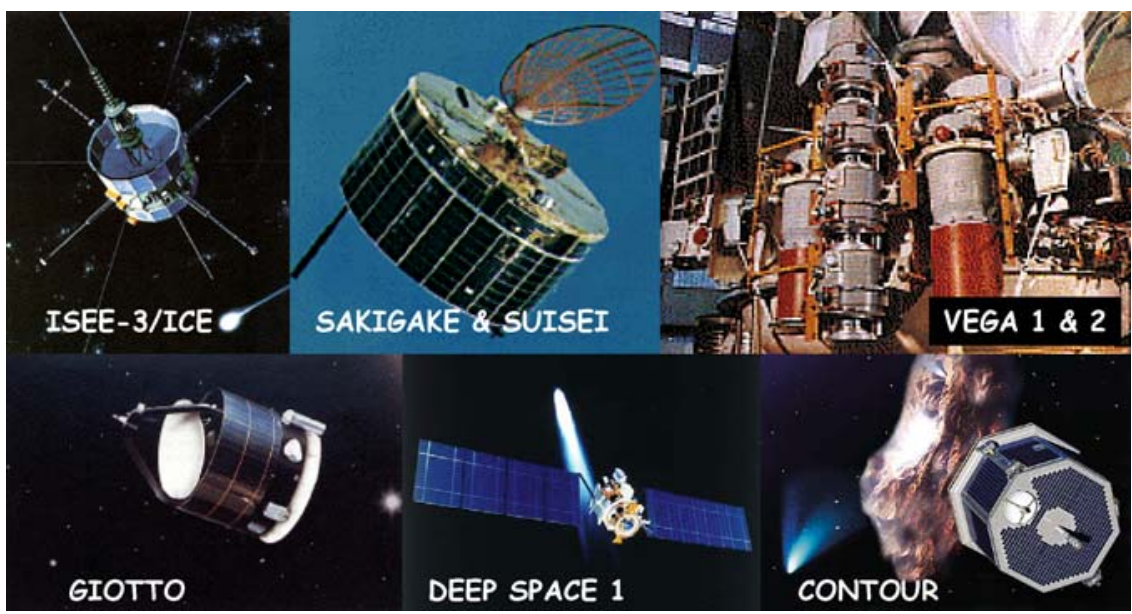

Fig. 13.7. Past cometary missions (courtesy of NASA)

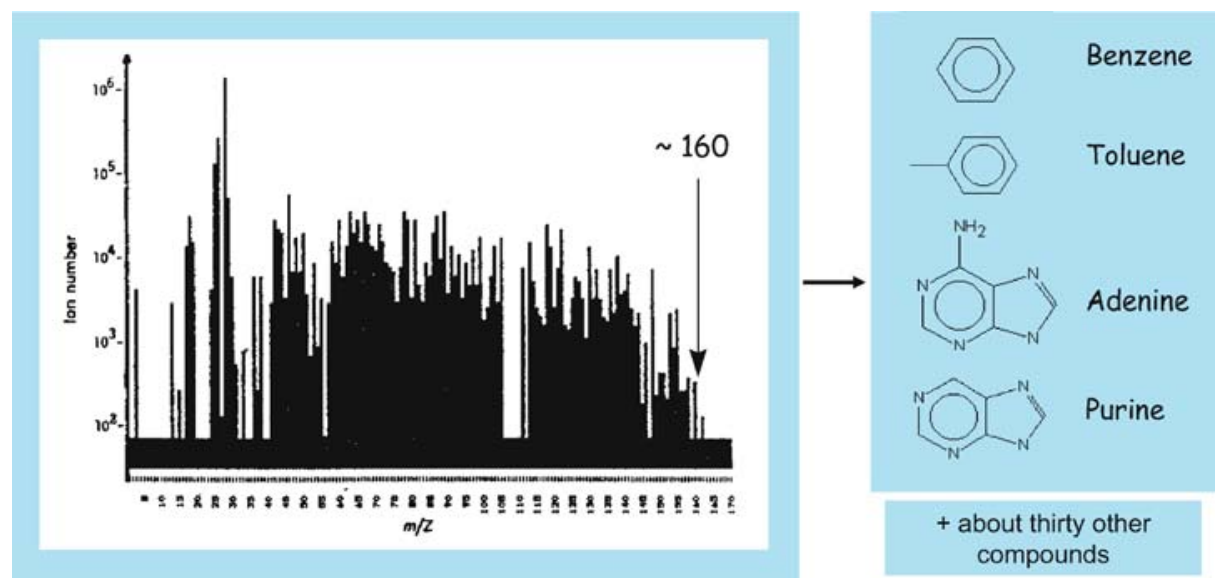

Fig. 13.8. Accumulated mass spectra collected in Comet Halley by the instrument PUMA on board Vega 1 spacecraft and tentative interpretation (adapted from Kissel and Krueger 1987)

been detected by mass spectrometry, mainly with the PICCA instrument (part of RPA) onboard the Giotto spacecraft, and the PUMA instrument, onboard Vega 1 . The first was dedicated to the analysis of the gaseous phase, whereas the latest was conceived to analyze the composition of cometary dust grains. The most fruitful related studies have been reported by Kissel and Krueger (1987). To interpret the PUMA mass spectra, they made hypotheses about the sudden energy dissipation processes near solid surfaces after an impact to infer the rules of molecular ion formation. Such impacts occur when a dust particle hits the mass spectrometer's target. This hypothesis yields a good agreement be- 
tween their predictions and other observations for small molecules (e.g., HCN, $\mathrm{CH}_{3} \mathrm{CN}$ ). For larger molecules, the problem is more complex, as they cannot survive the impact process without fragmentation. In addition to the difficulty of assembling correctly the fragments to reconstitute the original molecule, the nature of the fragments themselves is uncertain, several fragments having the same mass (at the spectrometer's resolution). Thus the identification by the authors of purines and adenine, for example, should be considered as very tentative. However, what is clearly shown by the PUMA mass spectra is that compounds with complex structure and large molecular masses (at least with $\mathrm{m} / \mathrm{z}$ up to $160 \mathrm{~g} \times \mathrm{mol}^{-1}$ ) are present in grains, which agrees with results from laboratory simulations.

On the other hand, first interpretations of the heavy-ion mass spectra from the PICCA instrument led Huebner (1987) to infer the presence of polyoxymethylene (POM, the formaldehyde polymer) in the Comet Halley. The alternation of patterns with a ratio $m / z=14$ and 16 is in good agreement with the succession of $-\mathrm{CH}_{2}-(m=14)$ and $-\mathrm{O}-(m=16)$ that form the polymer (Fig. 13.9). Huebner's hypothesis seemed to be confirmed by laboratory studies that measure POM mass spectra, which are in agreement with observations (Möller and Jackson 1990; Moore and Tanabe 1990). However, Mitchell et al. (1992) showed that the mass spectrum pattern which led Huebner to announce the detection of polyoxymethylene is only characteristic of a mixture of molecules composed of $\mathrm{C}, \mathrm{H}, \mathrm{O}$ and $\mathrm{N}$ atoms (CHON molecules). Therefore, PICCA mass spectra do not imply the presence of POM, but of a large diversity of CHON molecules, among which POM could be present. Moreover, measurements with the NMS (Neutral Mass Spectrometer) instrument, onboard Giotto, showed that

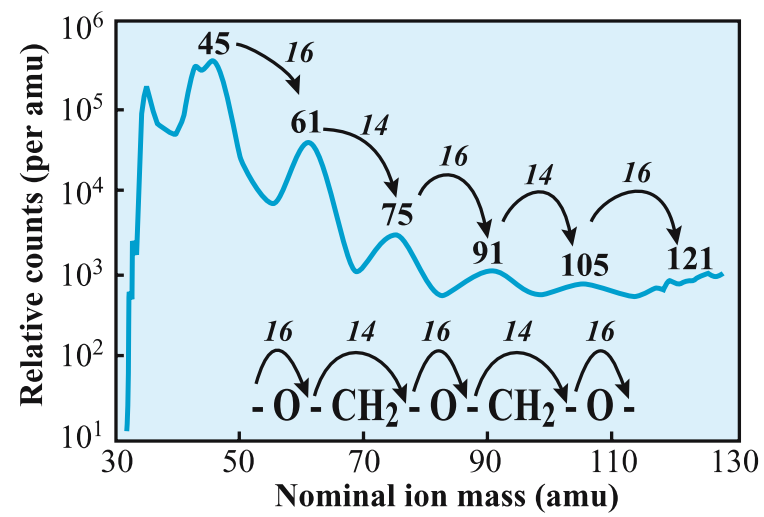

Fig. 13.9. Mass spectrum obtained with the PICCA instrument on board Giotto, March 14, 1986. This a mean of spectra acquired between 8200 and $12600 \mathrm{~km}$ from the nucleus. (Huebner 1987)). Peaks near $m / z=45$ are saturated, and masses beyond 120 are dominated by the apparatus noise. Comparison of the results with POM structure 


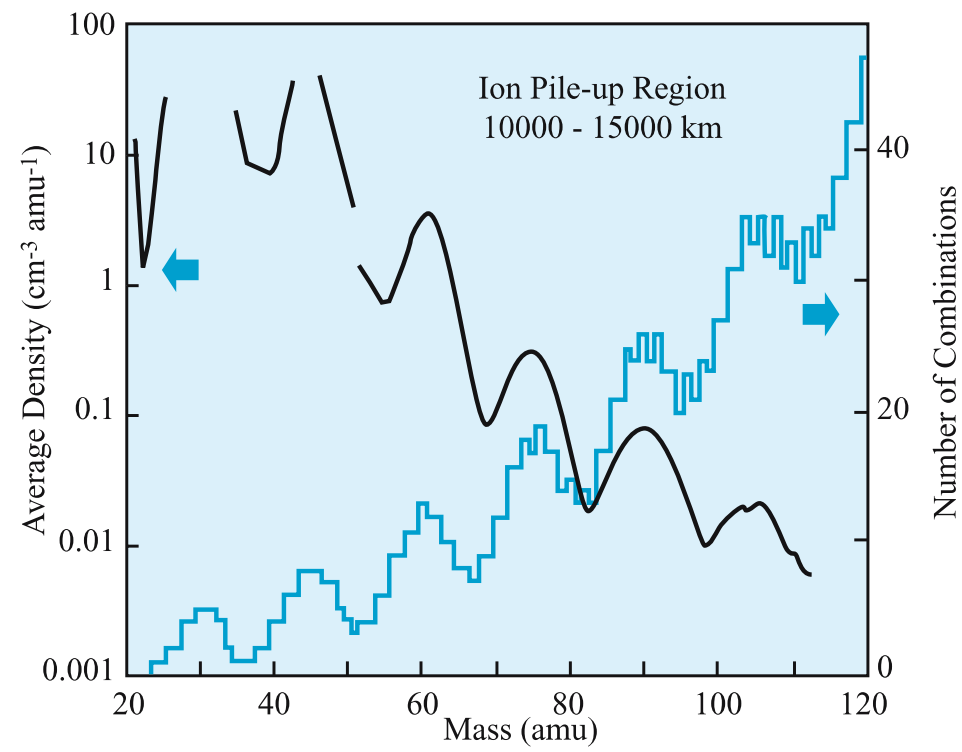

Fig. 13.10. PICCA mass spectrum compared to the number of combinations between C, H, O, N atoms leading to a molecular ion that can be observed (Mitchell et al. 1992)

the formaldehyde in Comet Halley could be produced by an extended source ${ }^{4}$ throughout the coma of Comet Halley (Meier et al. 1993). It has recently been shown that such observations can be explained by the presence of POM on grains (a few percents in mass). The polymer would be slowly decomposed into $\mathrm{H}_{2} \mathrm{CO}$ as it is heated and photolyzed (Cottin et al. 2004). Without being final evidence for the presence of POM in comets, this polymer is so far the best explanation to this extended source. Once it is imported to Earth's primitive oceans, it could be an interesting source of concentrated formaldehyde, and play a key role in the synthesis of prebiotic sugars. Table 13.4 shows all the molecules detected with mass spectrometry in Halley's vicinity. Those results, even if they are not always very specific, show that comets are extremely rich in terms of organic composition, and thus are of prime interest for astrobiology studies. Current and future missions have to take up the challenge of formal identification of complex molecules in comets.

\subsubsection{Current Missions}

In the infrared and radio wavelength domains, simple organic molecules have very specific signatures, and thus, they can be detected from the Earth by remote

${ }^{4}$ The $\mathrm{H}_{2} \mathrm{CO}$ spatial distribution in the coma is not compatible with an emission from the nucleus only. This supports the hypothesis that there is an (at least one) additional source which produces formaldehyde as it spreads outwards from the nucleus. This is called an extended source. 
Table 13.4. Organic molecules inferred from mass spectra of gas and dust particles in Comet Halley. The confidence levels of detections by MS are established as follows: Confirmed: molecule also detected by remote observations; High: molecule not detected by remote observations but present after laboratory irradiations of cometary ice analogues; Medium: molecule detected only by mass spectroscopy with a good confidence level according to the authors; Low: molecule only inferred by mass spectroscopy with a low confidence level according to the authors

\begin{tabular}{|c|c|c|c|c|}
\hline Molecule & Family & $\begin{array}{l}\text { Mass } \\
\text { spectrometer }\end{array}$ & $\begin{array}{l}\text { Confidence } \\
\text { level }\end{array}$ & Reference \\
\hline Hydrocyanic acid & $\mathrm{C}-\mathrm{N}-\mathrm{H}$ & PUMA, PICCA & Confirmed & $1,2,3$ \\
\hline Methyl cyanide & $\mathrm{C}-\mathrm{N}-\mathrm{H}$ & IMS & Confirmed & 7 \\
\hline Acetonitrile & $\mathrm{C}-\mathrm{N}-\mathrm{H}$ & PUMA, PICCA & Confirmed & $1,2,3$ \\
\hline Methanol & $\mathrm{C}-\mathrm{O}-\mathrm{H}$ & NMS & Confirmed & 6 \\
\hline Formaldehyde & $\mathrm{C}-\mathrm{O}-\mathrm{H}$ & PUMA, PICCA & Confirmed & 3 \\
\hline Formic acid & $\mathrm{C}-\mathrm{O}-\mathrm{H}$ & PUMA & Confirmed & 1,2 \\
\hline Acetaldehyde & C-O-H & PUMA, PICCA & Confirmed & 1,3 \\
\hline Ammonia & $\mathrm{N}-\mathrm{H}$ & PUMA & Confirmed & 2 \\
\hline Isocyanic acid & $\mathrm{C}-\mathrm{N}-\mathrm{O}-\mathrm{H}$ & PUMA & Confirmed & 1,2 \\
\hline Ethane & $\mathrm{C}-\mathrm{H}$ & NMS & Confirmed & 8 \\
\hline Acetylene & $\mathrm{C}-\mathrm{H}$ & NMS & Confirmed & 8 \\
\hline Acetic acid & $\mathrm{C}-\mathrm{O}-\mathrm{H}$ & PUMA & High & 1 \\
\hline Polyoxymethylene & $\mathrm{C}-\mathrm{O}-\mathrm{H}$ & PICCA & $\begin{array}{l}\text { High (itself } \\
\text { or derivatives) }\end{array}$ & 4,5 \\
\hline Ethene & $\mathrm{C}-\mathrm{H}$ & NMS & High & 8 \\
\hline Iminoethane & $\mathrm{C}-\mathrm{N}-\mathrm{H}$ & PUMA & Medium & 1,2 \\
\hline Aminoethene & $\mathrm{C}-\mathrm{N}-\mathrm{H}$ & PUMA & Medium & 1,2 \\
\hline Pyrroline & $\mathrm{C}-\mathrm{N}-\mathrm{H}$ & PUMA & Medium & 1 \\
\hline Pyrrole & $\mathrm{C}-\mathrm{N}-\mathrm{H}$ & PUMA & Medium & 1,2 \\
\hline Imidazole & $\mathrm{C}-\mathrm{N}-\mathrm{H}$ & PUMA & Medium & 1 \\
\hline Pyridine & $\mathrm{C}-\mathrm{N}-\mathrm{H}$ & PUMA & Medium & 1,2 \\
\hline Pyrimidine & $\mathrm{C}-\mathrm{N}-\mathrm{H}$ & PUMA & Medium & 1,2 \\
\hline Ethyl cyanide & $\mathrm{C}-\mathrm{N}-\mathrm{H}$ & IMS & Medium & 7 \\
\hline Pentyne & $\mathrm{C}-\mathrm{H}$ & PUMA & Low & 1 \\
\hline Hexyne & $\mathrm{C}-\mathrm{H}$ & PUMA & Low & 1 \\
\hline Butadiene & $\mathrm{C}-\mathrm{H}$ & PUMA & Low & 1 \\
\hline Pentadiene & $\mathrm{C}-\mathrm{H}$ & PUMA & Low & 1 \\
\hline Cyclopentene & $\mathrm{C}-\mathrm{H}$ & PUMA & Low & 1 \\
\hline Cyclopentadiene & $\mathrm{C}-\mathrm{H}$ & PUMA & Low & 1 \\
\hline Clyclohexene & $\mathrm{C}-\mathrm{H}$ & PUMA & Low & 1 \\
\hline Cyclohexadiene & $\mathrm{C}-\mathrm{H}$ & PUMA & Low & 1 \\
\hline
\end{tabular}


Table 13.4. (continued)

\begin{tabular}{lllll}
\hline Molecule & Family & $\begin{array}{l}\text { Mass } \\
\text { Spectrometer }\end{array}$ & $\begin{array}{l}\text { Confidence } \\
\text { Level }\end{array}$ & Reference \\
\hline Benzene & C-H & PUMA & Low & 1 \\
Toluene & C-H & PUMA & Low & 1 \\
Propanenitrile & C-N-H & PUMA & Low & 1 \\
Iminomethane & C-N-H & PUMA & Low & 1 \\
Iminopropene & C-N-H & PUMA & Low & 1 \\
Purine & C-N-H & PUMA & Low & 1,2 \\
Adenine & C-N-H & PUMA & Low & 1,2 \\
Polyaminocyano- & C-N-H & PICCA & Low & 5 \\
methylene & & & & 1 \\
Methanolnitrile & C-N-O-H & PUMA & Low & 1 \\
Methanalimine & C-N-O-H & PUMA & Low & 1 \\
Aminomethanol & C-N-O-H & PUMA & Low & 2 \\
Aminomethanal & C-N-O-H & PUMA & Low & 2 \\
Oxyimidazole & C-N-O-H & PUMA & Low & 1 \\
Oxypyrimidine & C-N-O-H & PUMA & Low & 1 \\
Xanthine & C-N-O-H & PUMA & Low & 1 \\
\hline
\end{tabular}

1 (Kissel and Krueger 1987); 2 (Krueger and Kissel 1987); 3 (Krueger et al. 1991); 4 (Huebner 1987); 5 (Huebner et al. 1989); 6 (Eberhardt and Krankowsky 1995); 7 (Geiss et al. 1999); 8 (Altwegg et al. 1999)

sensing. This is not the case for complex compounds, for which low abundances and/or less specific spectroscopic signatures requires other investigation means. This can be illustrated by the three current space missions dealing with comets: Stardust (sample return from comet Wild 2), Deep Impact (impact with comet Temple 1 nucleus) and Rosetta (aiming to intercept comet 67P/ChuruymovGerasimenko, and for which one of the instruments is a gas chromatograph).

Information about those missions is presented in Table 13.5 and Fig. 13.11. Stardust mission consists in two phases: collecting cometary dust grains with an aerogel, which is now completed, then the analysis of the grains in Earth laboratories after recovering the samples in January 2006. As far as the analysis of the molecular composition of grains and their astrobiological relevance are concerned, most of the science part will be done after the sample returns. Yet, it is interesting to note that evidences of the presence of complex organic material on grains have already been obtained thanks to the mass spectrometer onboard Stardust (CIDA instrument) (Kissel et al. 2004). However, those analyses have been performed on grains that may have evolved since their release from the 


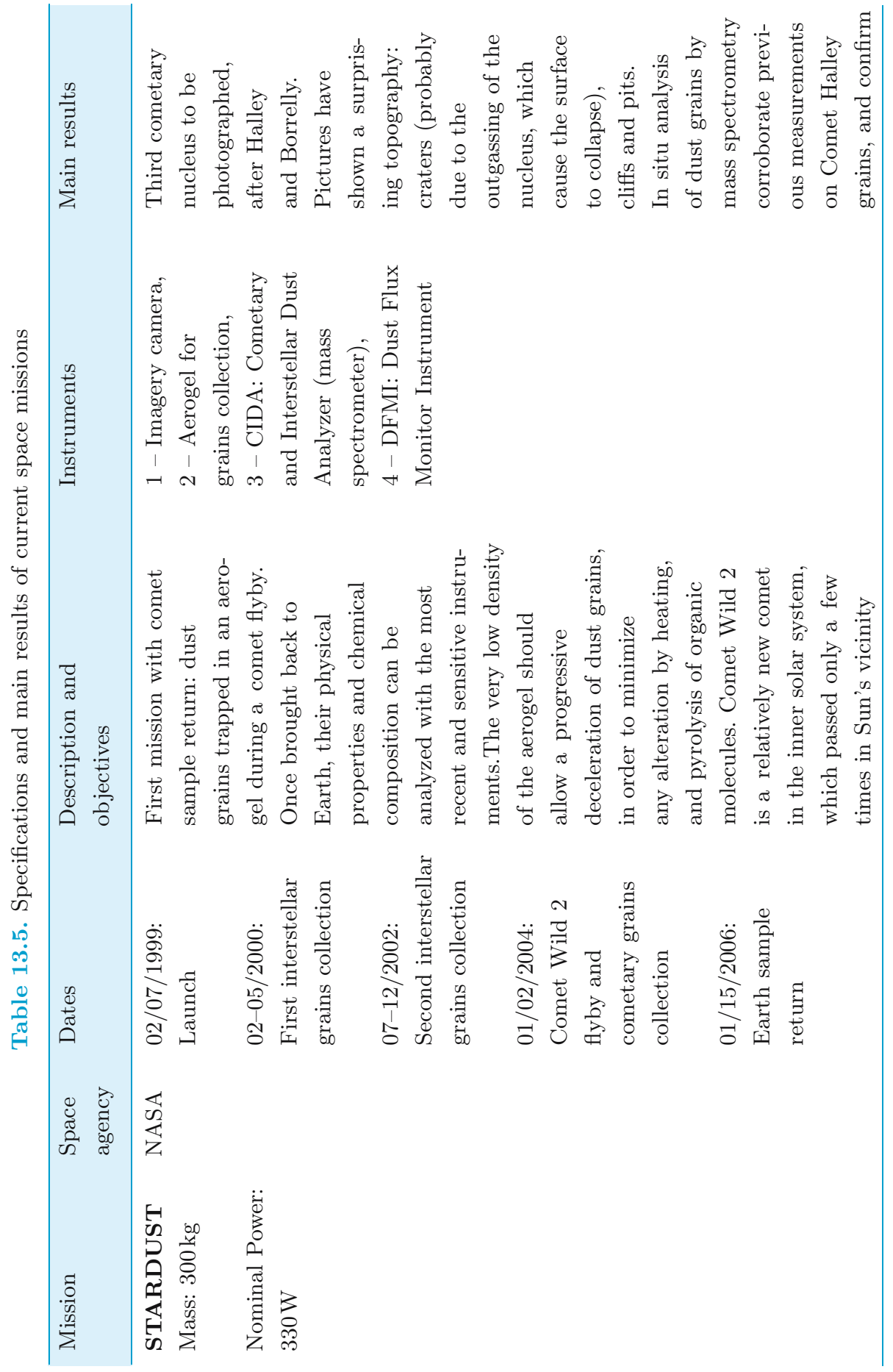




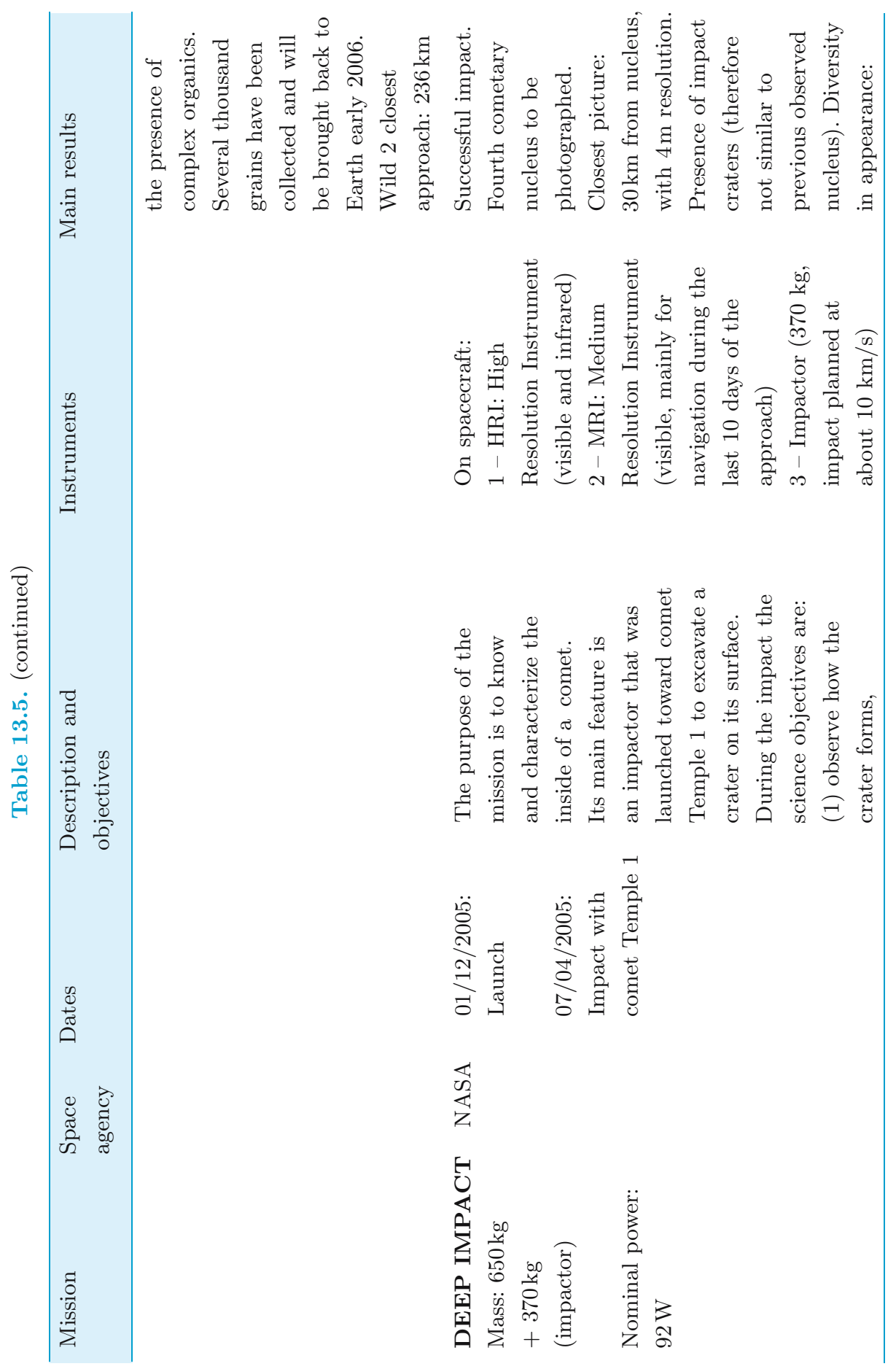




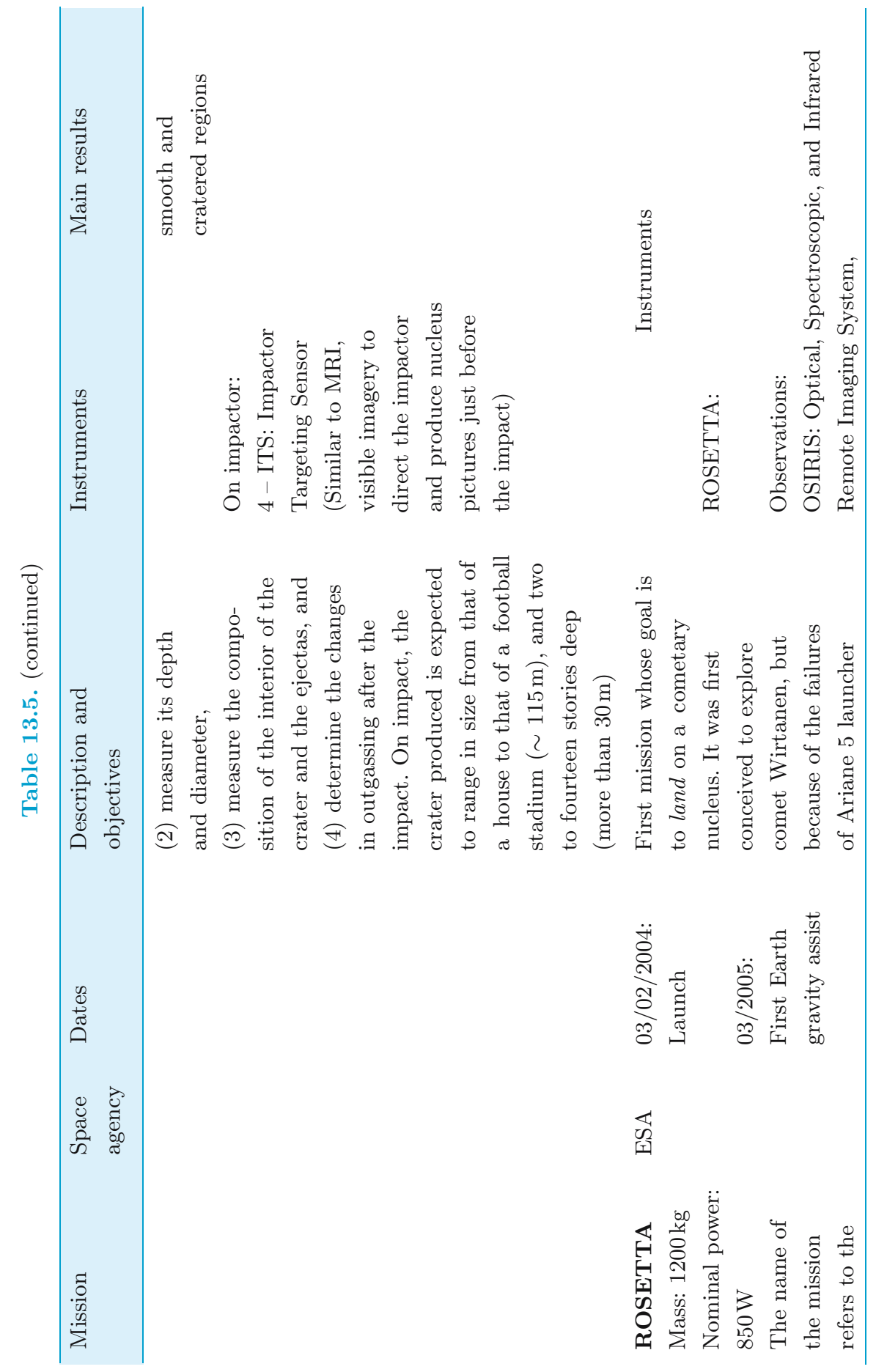




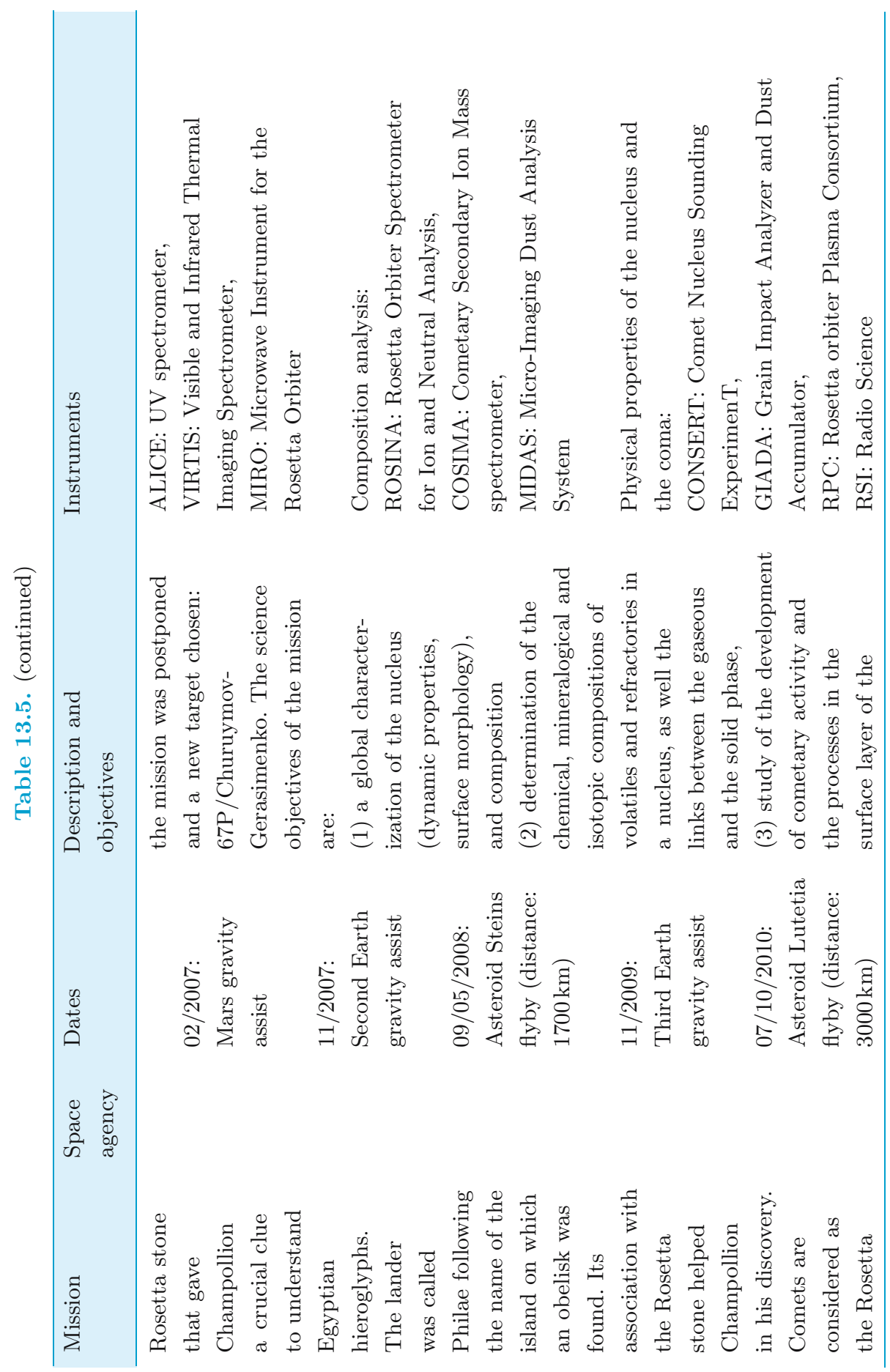




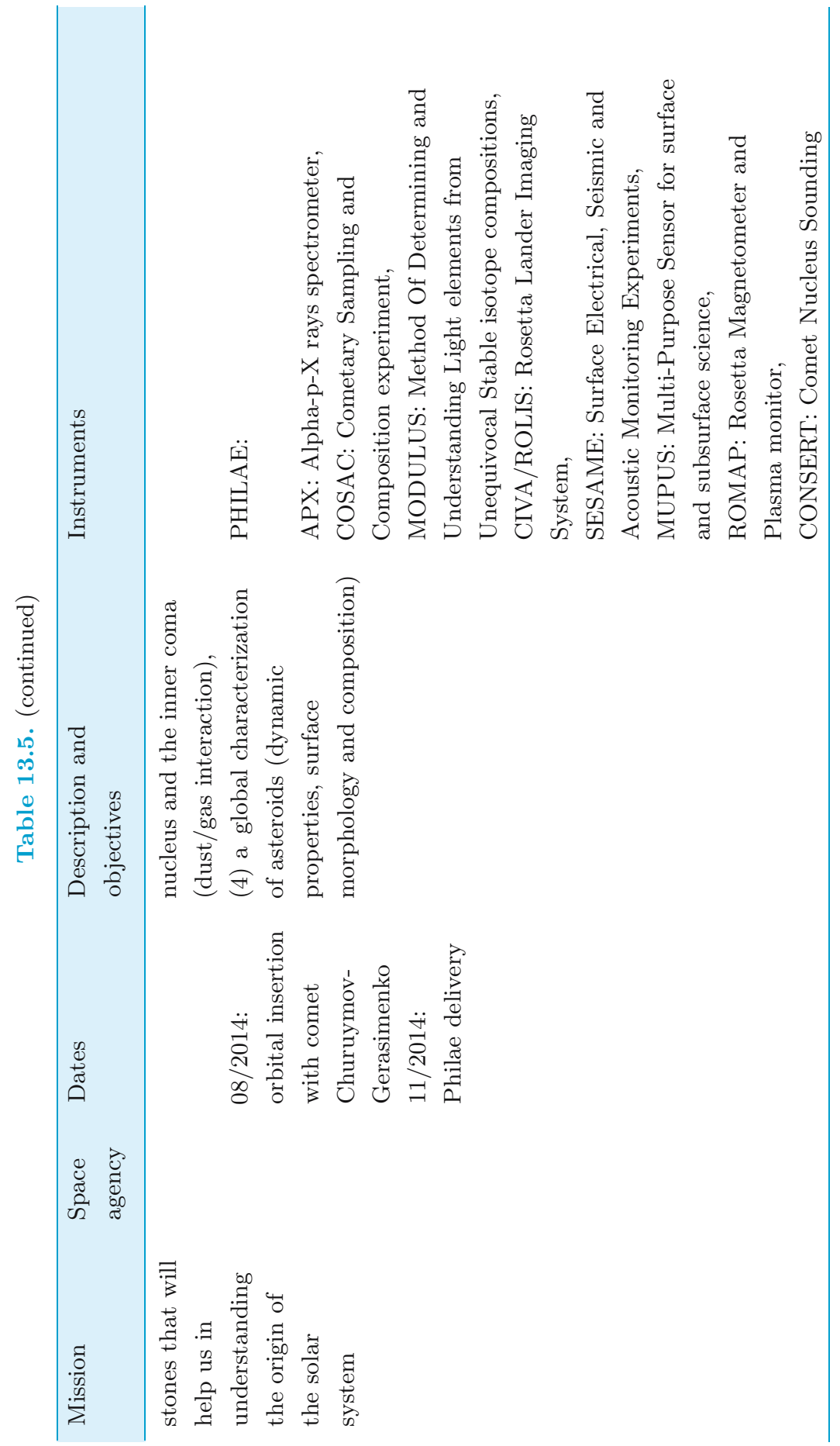




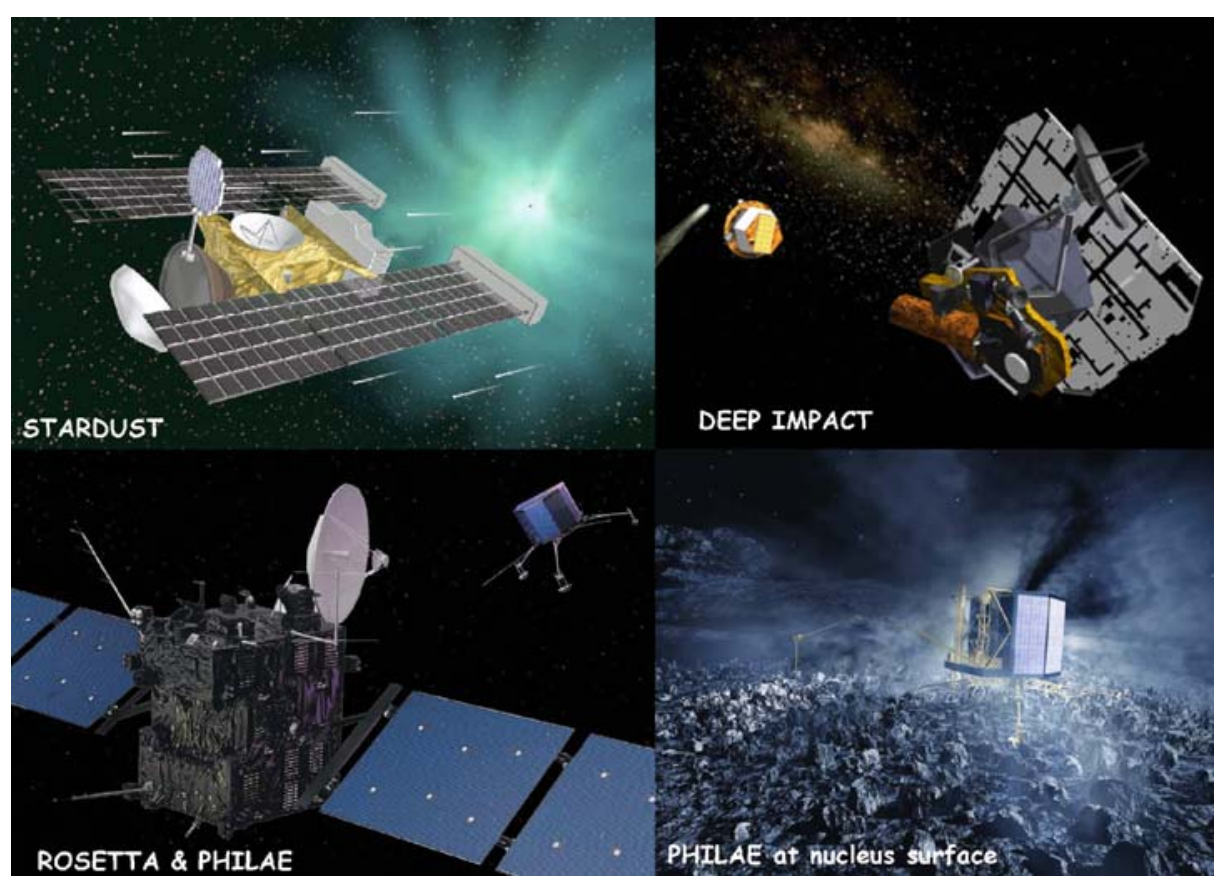

Fig. 13.11. Current space missions (courtesy of NASA \& ESA)

nucleus, and for the grains that will be analyzed on Earth, one cannot rule out some alteration when impacting the aerogel. Therefore, measurements directly on the nucleus are necessary.

Deep Impact mission aims to gain access to the interior of a comet, and then observe what could be the most pristine matter of the solar system, since cometary inside layers have been protected from irradiation and heating due to previous Sun approaches. A collision between an impactor and comet Temple 1 was planned on July 4, 2005, in order to excavate a large crater at the surface. The impact was a real success as can be seen in Fig. 13.12. At the time this chapter was written, only imagery data has been released, therefore we cannot discuss further the results in terms of nucleus properties and chemical composition. It has only been shown that there will be almost no change in the cometary trajectory because of the Deep Impact collision. New chemical species may be detected during the increase of outgassing after the impact, either with the onboard infrared instrument, or with other remote sensing instruments on Earth. Many new data concerning comets are excepted when the results of this mission become published.

As for the Rosetta mission, it consists of two parts: one orbiter revolving around the nucleus, and a lander called Philae. The instruments onboard the orbiter will enable an unprecedented analysis of the composition of volatile and 


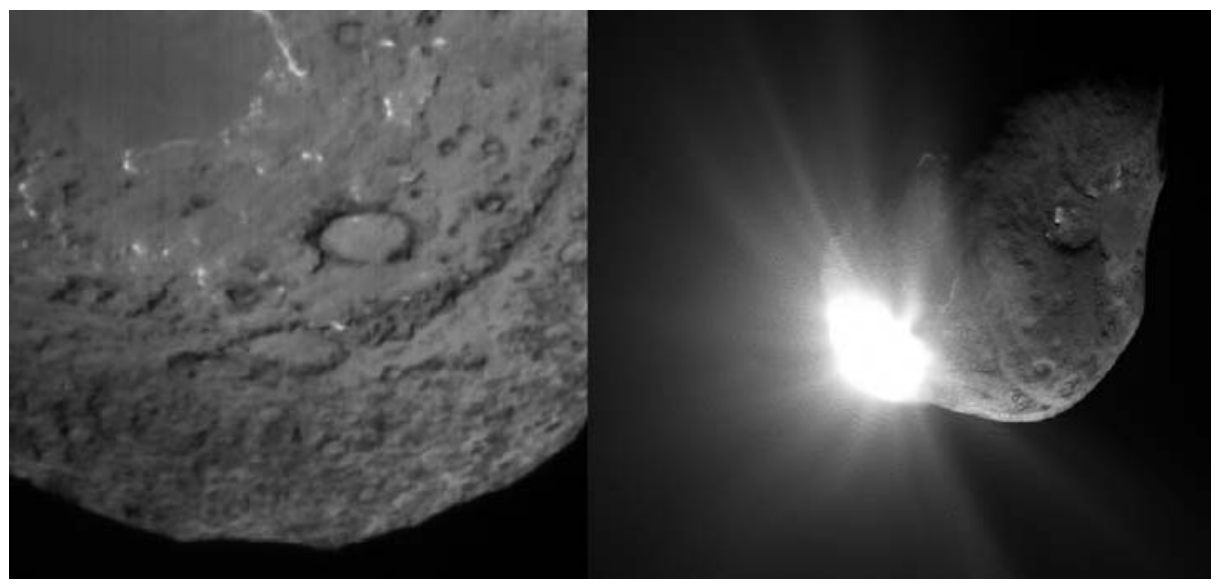

Fig. 13.12. Cometary nucleus 90 seconds before the impact seen from the impactor at $900 \mathrm{~km}$ ( on the left) and impact event seen from the spacecraft (on the right). (courtesy of NASA/JPL-Caltech/UMD)

refractory compounds released from the nucleus. Three instruments (ROSINA, COSIMA and MIDAS) will collect and analyze gas and dust as close as $1 \mathrm{~km}$ from the surface of the nucleus, it is expected that they will be close enough to study almost unaltered matter compared to its release from the nucleus. Nevertheless, probably the most fruitful information from an astrobiological point of view will come from Philae. In this chapter, we have decided to emphasize the COSAC instrument since most of the new organic molecules should be detected thanks to this experiment.

The COSAC instrument is a gas chromatograph (GC) coupled with a mass spectrometer (MS, a linear time of flight spectrometer in this case). It consists of 8 chromatographic columns, each of them being connected to its own detector (TCD), but it is also possible to connect them to a mass spectrometer (Rosenbauer et al. 1999) (Fig. 13.13). Previous results obtained thanks to direct mass spectrometry measurements with Puma, Giotto and Stardust spacecrafts, gave only the mass spectrum of the mixture of all the molecules at the same time. COSAC will carry out a preliminary separation by chromatography, which will achieve a quasidefinite identification of the compounds since they will be identified both from their retention time ${ }^{5}$, and from their individual mass spectra. Samples will be collected after drilling the surface and heated at various temperatures before being injected into the analysis system. Pyrolysis (up to $600^{\circ} \mathrm{C}$ ) is possible in order to degrade the most refractory component, and then enable gas phase analysis of the fragments. Out of the eight chromatographic

${ }^{5}$ The time for a compound to be eluted from the chromatographic column, this time depends on the properties of both the molecules and the stationary phase of the column. 


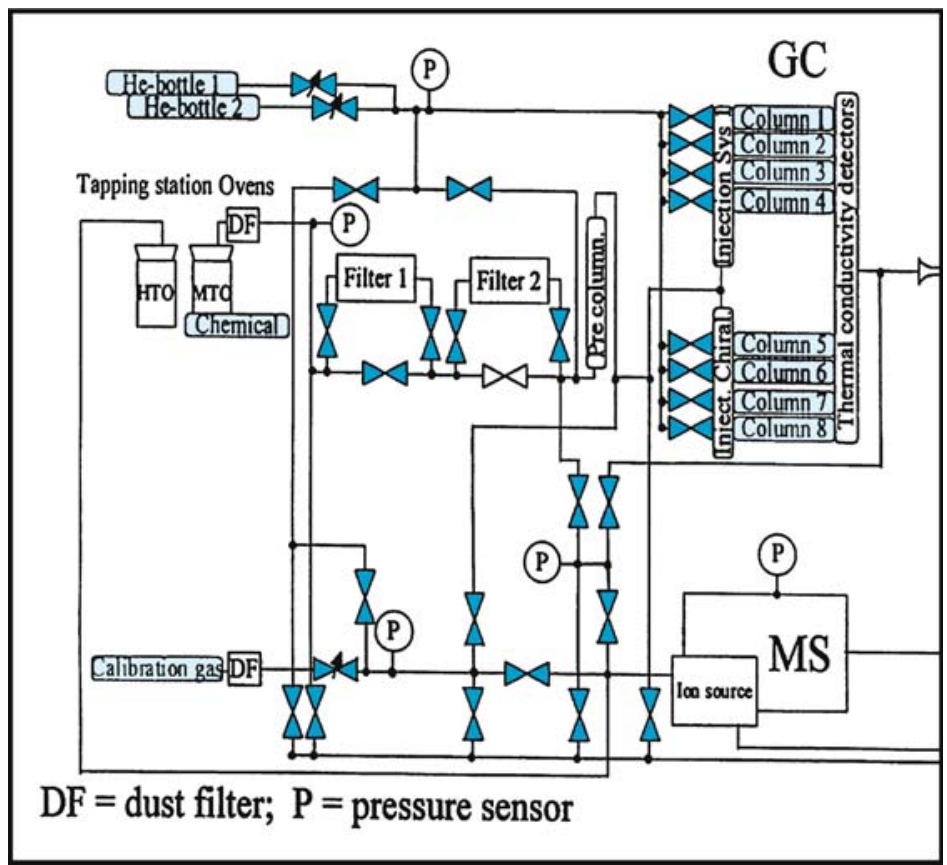

Fig. 13.13. The COSAC experiment (Rosenbauer et al. 1999)

columns, three are specifically devoted to the analysis of chiral molecules in order to distinguish enantiomers. The other five columns have been selected so that a maximum of molecules can be detected. Moreover, the simultaneous analysis of a single sample with several columns (up to four columns at the same time) will facilitate the data analysis by comparison, and thus increase the interpretation's reliability. Table 13.6 shows all the molecules that were considered for the selection of the chromatographic columns, and the ones that will be actually possible to detect. One can note that amino acids and other heavy compounds such as oxalic acid, urea, etc., are not detectable, since they are not volatile enough to be analyzed in the gaseous phase. GC analysis of such compounds requires a preliminary stage called derivatization (chemical reaction that makes the targeted compound more volatile). This procedure is not feasible with the COSAC instrument, but work is in progress to include derivatization in future Martian exploration experiments (Rodier et al. 2001). Nucleus analyses by the COSAC instrument will be completed by CIVA (infrared analysis) and MODULUS (for isotopic measurements).

It is interesting to note that HMT, a molecule that is very often produced during laboratory simulations of cometary ices (Bernstein et al. 1995; Cottin et al. 2001; Muñoz Caro and Schutte 2003), will be detected by the COSAC instrument if it is present on the nucleus. Indeed, even if this compound is 
Table 13.6. Organic molecules that might be detected in comets thanks to the COSAC instrument (Szopa et al. 2003)

List of the compounds considered for the study (Abbreviations: d: detected in comac; s: gencrated during expcrimental simulations; a: analysable by the $\operatorname{COSAC~GC~subsystem~within~the~space~conditions:~p:~potentially:~italic:~compounds~not~vaporisable~without~decomposition)~}$

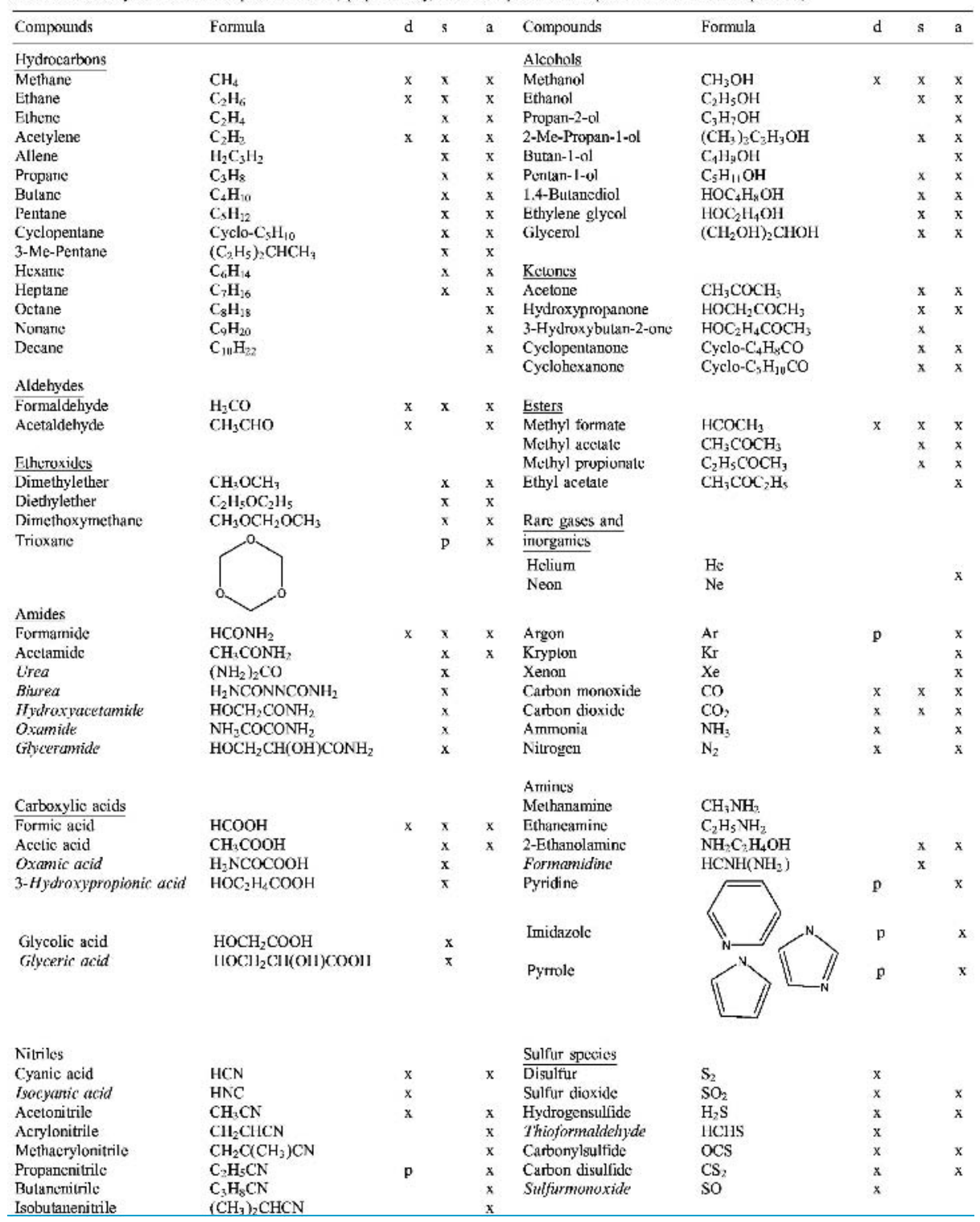


Table 13.6. (continued)

\begin{tabular}{|c|c|c|c|c|}
\hline Compounds & Formula & $\mathrm{d}$ & s & a \\
\hline Cis-crotonitrile & cis- $\mathrm{CH}_{3} \mathrm{C}_{2} \mathrm{H}_{2} \mathrm{CN}$ & & & $\mathrm{x}$ \\
\hline Trans-crotonitrile & trans- $\mathrm{CH}_{3} \mathrm{C}_{2} \mathrm{H}_{3} \mathrm{CN}$ & & & $x$ \\
\hline \multicolumn{5}{|l|}{ Amino acids } \\
\hline Glycine & $\mathrm{NH}_{2} \mathrm{CH}_{2} \mathrm{COOH}$ & & $x$ & \\
\hline Alunine & $\mathrm{CH}_{3} \mathrm{CH}\left(\mathrm{NH}_{2}\right) \mathrm{COOH}$ & & $x$ & \\
\hline$\alpha-A B A$ & $\mathrm{C}_{2} \mathrm{H}_{5} \mathrm{CH}\left(\mathrm{NH}_{2}\right) \mathrm{COCOH}$ & & $x$ & \\
\hline$B-A B A$ & $\mathrm{CH}_{3} \mathrm{CH}\left(\mathrm{NH}_{2}\right) \mathrm{CH}_{2} \mathrm{COOH}$ & & $x$ & \\
\hline$A I B A$ & $\left(\mathrm{CH}_{2} \mathrm{NH}_{2}\right)\left(\mathrm{CH}_{3}\right) \mathrm{CHCOOH}$ & & $\mathrm{x}$ & \\
\hline
\end{tabular}

Aromatics

Benzene

Toluene

Phenylacetylene

Benzeneacetic acid

Indenc

Quinoline

2-Me-Naphthalene

Naphthalene

Anthracene

phenanthrene

Dioltetrahyloro-

anthracene

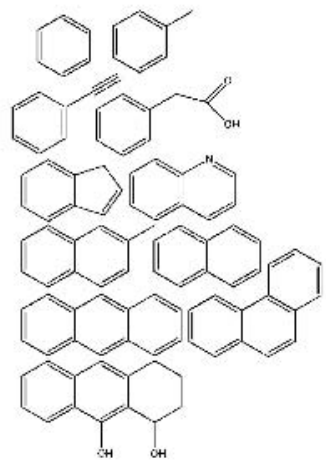

$\begin{array}{ccc}\mathrm{p} & & \mathrm{x} \\ \mathrm{p} & & \mathrm{x} \\ & \mathrm{x} & \mathrm{x} \\ & \mathrm{x} & \mathrm{x} \\ \mathrm{x} & \mathrm{x} \\ \mathrm{x} & \mathrm{x} \\ \mathrm{x} & \mathrm{x} \\ & & \mathrm{x}\end{array}$

\begin{tabular}{|c|c|c|c|c|}
\hline Compounds & Formula & $\mathrm{d}$ & s & a \\
\hline Others & & & & \\
\hline Polyoxymethylene & $\left(-\mathrm{CH}_{2}-\mathrm{O}-\right)_{n}$ & $\mathrm{p}$ & $x$ & \\
\hline Hydrazine & $\mathrm{N}_{2} \mathrm{H}_{4}$ & & $x$ & \\
\hline Carbonic acid & $\mathrm{H}_{2} \mathrm{CO}_{3}$ & & $\mathrm{x}$ & \\
\hline Hydrogen Peroxide & $\mathrm{H}_{2} \mathrm{O}_{2}$ & & $x$ & \\
\hline Curbon suboxide & $O \mathrm{CC}_{3} \mathrm{O}$ & & $\mathrm{x}$ & \\
\hline Isocyonic acid & HINCO & $\mathrm{x}$ & $\mathrm{x}$ & \\
\hline Water & $\mathrm{H}_{2} \mathrm{O}$ & $x$ & & $x$ \\
\hline Hexamethylenetetramine & & & $x$ & $x$ \\
\hline
\end{tabular}

easily synthesized in the conditions simulated in the laboratory, it remains undetected in comets or interstellar medium. HMT could be a source of amino acids once imported to Earth, since its hydrolysis yields the formation of amino acids (Wolman et al. 1971). Its actual detection in comets would validate the contribution of laboratory experimental simulation to understanding the chemistry of cometary ices. On the other hand, COSAC will not be able to detect POM (see Sect. 13.2.2) because it is readily degraded into formaldehyde when heated. Infrared measurements might be sufficient to detect it, but the spectroscopic signatures of this compound could be overlapped by other high molecular weight organic molecules.

Thus, our knowledge in terms of molecular composition of comets will do a great leap forward at the time of Rosetta's arrival near comet 67P/Churuymov- 
Table 13.7. Some data about comet 67P/Churyumov-Gerasimenko

Discovery: 1969 by Klim Churyumov and Svetlana Gerasimenko

Orbital period: $6.57 \mathrm{yrs}$

Aphelion: 858 million km, i.e., $5.73 \mathrm{AU}$

Perihelion: 194 million km, i.e., 1.3 AU

Mean nucleus diameter: $1980 \mathrm{~m}$

Gerasimenko. With the new detected molecules, we will be in a better position to evaluate the parameters necessary to their production, and then trace back the physicochemical condition prevailing in the solar system when the comets were accreted, or even better, to the composition of our natal molecular cloud. Therefore, the astrobiology relevance of the comet will be disproved or strengthened.

\subsubsection{Future Space Missions}

The next step of comets exploration will probably be a return of samples taken at the surface of a nucleus (and underneath). There is not yet such a mission under development, but proposals have already been submitted to NASA and nucleus sample return missions are to be expected to be accepted in the years to come.

As already mentioned earlier in this chapter, knowing the composition of one comet will not allow us to extrapolate the results to the whole family of comets, as their composition should depend on the place they were formed (see Fig. 13.3). Then, one shall vary our targets selections (from the Kuiper Belt, the Oort Cloud, few or many perihelion passages), before pretending to know these mysterious objects.

\subsection{The Space Exploration of Titan}

\subsubsection{Observations and Models of Titan Before Space Missions}

On the night of March 25, 1655, using a refracting telescope with a focal length of almost four meters and a magnifying power of 50, Christiaan Huygens observed for the first time a small star aligned with the rings of Saturn at a distance of three arc seconds. ${ }^{6}$ During the following days, he made new observations and noted the displacement of this object as compared to fixed stars and Saturn. This star followed Saturn's course in the sky, and thus Huygens deduced that it must be a satellite. He had just discovered the moon Titan.

In 1944, making spectroscopic measurements of Titan in the atmospheric window between 550 and $850 \mathrm{~nm}$, Kuiper observed for the first time the methane

\footnotetext{
${ }^{6}$ Angular distance projected on the sky.
} 


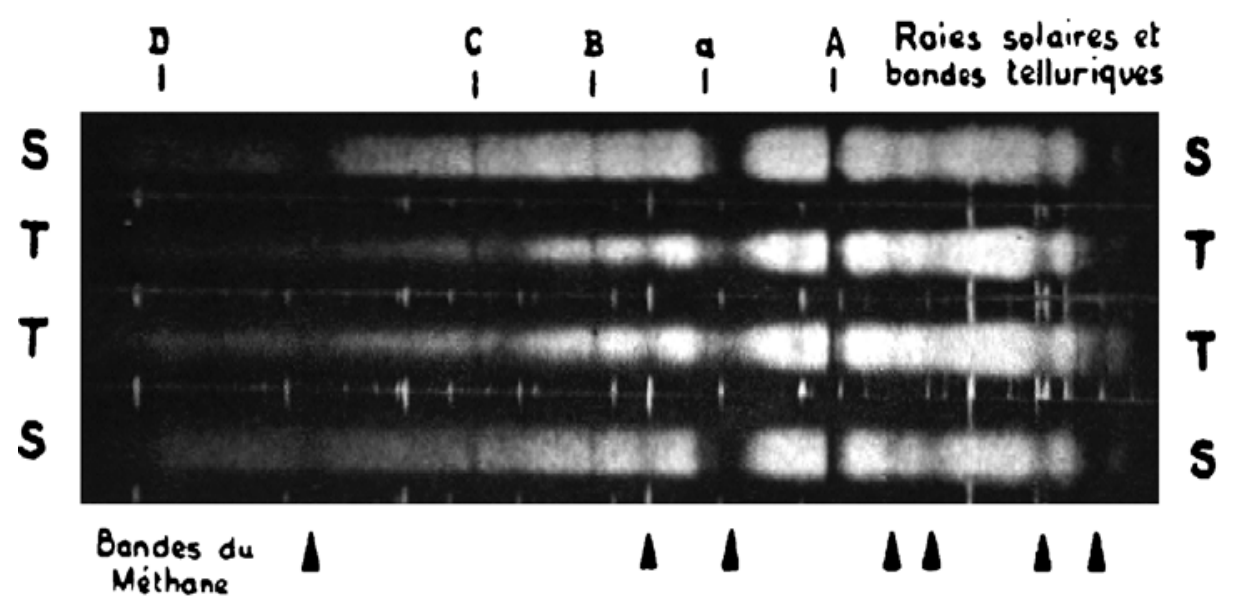

$\begin{array}{lcc}\text { Band } & \text { Wavelenght }(\AA) & \frac{\text { Origin }}{\mathrm{O}_{2}} \\ \text { A } & 7594 & \mathrm{H}_{2} \mathrm{O} \\ \text { a } & 7187 & \mathrm{O}_{2} \\ \text { B } & 6867 & \mathrm{H \alpha} \\ \text { C } & 6563 & \mathrm{Na}\end{array}$

Fig. 13.14. Saturn $(S)$ and Titan $(T)$ spectra obtained by Kuiper, at the MacDonald observatory (1944) showing characteristic methane absorption bands between 5500 and $9000 \AA$. The wavelength calibration is given by the Fraunhofer ${ }^{7}$ and telluric absorption bands

absorption bands (Fig. 13.14). In 1965, Low (1965) made the first measurements of the flux emitted from Titan in the $10 \mu \mathrm{m}$ infrared atmospheric window. He determined a brightness temperature ${ }^{8}$ of $\sim 160 \mathrm{~K}$. However, it is only in the beginning of the 1970s that the development of space missions and infrared detectors allowed the discovery of the extraordinary rich organic chemistry occurring in the atmosphere of this satellite.

From infrared observations obtained in the $8-14 \mu \mathrm{m}$ window, Gillett et al. (1973) attributed the emissions bands to the $\nu_{4}$ methane band and the $\nu_{7}$ ethane band. At the same time, Trafton (1972a,b) detected traces of molecular hydrogen. On their side, Veverka (1973) and Zellner (1973) showed, analyzing polarimetric observational data, the presence of a dense and highly diffusive layer in the atmosphere. Finally, Caldwell (1975) using the OAO-2 (Orbital Astronomical Observatory) satellite, obtained photometric measurements in the ultraviolet wavelength range, around $260 \mathrm{~nm}$ and inferred a very low albedo, on the order of 0.05 .

\footnotetext{
${ }^{7}$ Absorption lines visible in the solar spectra.

8 Temperature of an object considered as a black body.
} 
The infrared band of methane and ethane are observed in emission, which can be explained by a temperature inversion in the atmosphere. Titan's atmospheric models have this inversion together with the high brightness temperature, the low ultraviolet albedo and the polarimetric measurements. A first set of models, developed initially by Pollack (1973), included a dense cloudy highly diffusive layer that could explain the visible spectrum and polarimetric data. A greenhouse effect that could be produced by induced absorption of a 0.5 bar equimolar $\mathrm{H}_{2}$ and $\mathrm{CH}_{4}$ mixture, would explain the $160 \mathrm{~K}$ temperature. Nevertheless, this model does not account for the low ultraviolet albedo and the temperature inversion. Consequently, a second type of model had to be constructed. Since the Rayleigh diffusion (diffusion by the gas) cannot explain the low albedo, Danielson et al. (1973) proposed that small particles ("dust"), efficiently absorbing ultraviolet photons, could be present in the high atmosphere. This hypothesis is supported by the fact that at the same period Khare and Sagan (1973) produced in their simulation experiment (photon irradiation of a $\mathrm{NH}_{3}-\mathrm{CH}_{4}-\mathrm{H}_{2}$ mixture) a brown polymer that had similar properties compared to Danielson dust. Thus, if such material would be produced in the high atmosphere of Titan, these aerosols would have a very small size and could not emit efficiently the absorbed photons. Consequently, their temperature would increase until their emission equilibrates the absorbed energy. The temperature of the gas would then increase through collision with those aerosols up to the observed $160 \mathrm{~K}$. In this model, the surface would have a black body temperature of $80 \mathrm{~K}$.

The two models are very different but a compromise seems possible. Hunten (1978) proposed a model in which the low atmosphere is described by the first model and the high atmosphere by the second model. Taking into account the low gravity on $\operatorname{Titan}^{9}$, the hydrogen amount proposed by Pollack (1973) is not realistic; it is therefore replaced by molecular nitrogen. The choice of $\mathrm{N}_{2}$ comes from the studies about the initial composition of Titan. Nitrogen could have been trapped in the ices in the form of ammonia that could then produce molecular nitrogen photolysis. Atreya et al. (1978) calculated that, if Titan had an important greenhouse effect at the beginning of its history $(T>150 \mathrm{~K}), \mathrm{NH}_{3}$ could have lead to the formation of 20 bars of $\mathrm{N}_{2}$. Hunten's model includes $\mathrm{N}_{2}$ as the main constituent (with $0.25 \% \mathrm{CH}_{4}$ and $0.5 \% \mathrm{H}_{2}$ ), a cloud with a highly diffusing upper part, and a high atmosphere containing dust. In this model, the tropopause is determined by the altitude where the optical depth $\left(\mathrm{N}_{2}\right.$ pressure induces absorption opacity plus cloud opacity) equals one. This region of the atmosphere is supposed to have an effective temperature of $77 \mathrm{~K}$ and a pressure of $\sim 600 \mathrm{mbar}$. The surface pressure is then deduced from the adiabatic approximation from the tropopause down to the ground. This leads to a surface pressure of $\sim 20$ bars for a surface temperature of $200 \mathrm{~K}$ compatible with the millimeter observations (Conklin et al. 1977). Nevertheless, radio observations in the centimeter domain where the atmosphere is supposed to be transparent, lead to brightness temperature of $100 \mathrm{~K}$. The main uncertainty on this measurement

\footnotetext{
${ }^{9}$ Low gravity favors light element such as hydrogen to escape from the atmosphere.
} 
comes from the poor angular resolution, which could lead to confusion between Titan and Saturn. Still, as pointed out by Hunten, his model could also lead to a lower temperature: for example, a $100 \mathrm{~K}$ surface temperature would lead to a 2-bar surface pressure.

Finally, one can note that the photochemical model developed by Strobel (1974) with an atmosphere mainly composed of $\mathrm{CH}_{4}$ and $\mathrm{H}_{2}$ in same quantities (this model was initially developed for Jupiter with $\mathrm{CH}_{4} / \mathrm{H}_{2} \ll 1$ ), predicts the production of $\mathrm{C}_{2} \mathrm{H}_{6}, \mathrm{C}_{2} \mathrm{H}_{2}$, and in smaller amounts $\mathrm{C}_{2} \mathrm{H}_{4}$, and $\mathrm{C}_{3} \mathrm{H}_{4}$ as trace constituents. The presence of $\mathrm{C}_{2} \mathrm{H}_{2}$ and $\mathrm{C}_{2} \mathrm{H}_{4}$ has been later confirmed by observational data obtained by Gillett (1975) in the 8 to $14 \mu \mathrm{m}$ wavelength range. $\mathrm{CH}_{3} \mathrm{D}$ was also detected at that time.

\subsubsection{Voyager Missions at Titan}

Launched in 1977, the Voyager 1 and 2 spacecrafts arrived in the Saturn system three years later after a flyby of Jupiter in 1979, where they obtained a large amount of data. The Saturn encounter was planned so that the maximum information could be gathered about the rings, the satellites and the magnetosphere, and to allow the study of Saturn's and Titan's atmospheres. Titan studies focused on several major goals: determine the diameter of the solid surface, the temperature and pressure profiles, the atmospheric composition and search for a magnetic field (Stone and Miner 1981).

Voyager 1 spacecraft obtained the largest amount of data on Titan. In fact, its trajectory was planned to approach the satellite as much as it could and its closest approach was at less than $7000 \mathrm{~km}$ from the center of Titan (Ness et al. 1981). A first disappointment came from the pictures obtained (Smith et al. 1981). In fact, even if the presence of aerosol was expected, some scientists were hoping that some hole in the haze would allow for the observation of the surface. It was not the case. A uniform orange haze was entirely hiding the surface. Nevertheless, the images showed three distinct aerosol layers (Fig. 13.15). The first one was observed around a $200 \mathrm{~km}$ altitude. It was dense, optically thick in the visible and seemed to be made of particles having a $\sim 0.3 \mu \mathrm{m}$ radius at the top. A second layer detached from the first one (even if a small amount of aerosols was observed in between) was seen $100 \mathrm{~km}$ above and was about $50 \mathrm{~km}$ thick. The last layer was observed at a mean altitude of $400 \mathrm{~km}$ and was also observed by the ultraviolet occultation experiment. Finally, the observations showed a northsouth asymmetry, the north being much darker than the southern hemisphere.

The greatest progress in our knowledge of this satellite of Saturn came from radio and ultraviolet occultation measurements and from infrared and ultraviolet experiments. The radio occultation experiment $(\sim 3.6$ and $13 \mathrm{~cm})$ sounded the atmosphere (Tyler et al. 1981) down to the surface allowing the determination of the radius of $2575.0 \pm 0.5 \mathrm{~km}$ (Lindal et al. 1983). This type of sounding measures the variation of the refractive index of the atmosphere as a function of altitude. Since the refractivity is proportional to the density, this allows to determine 


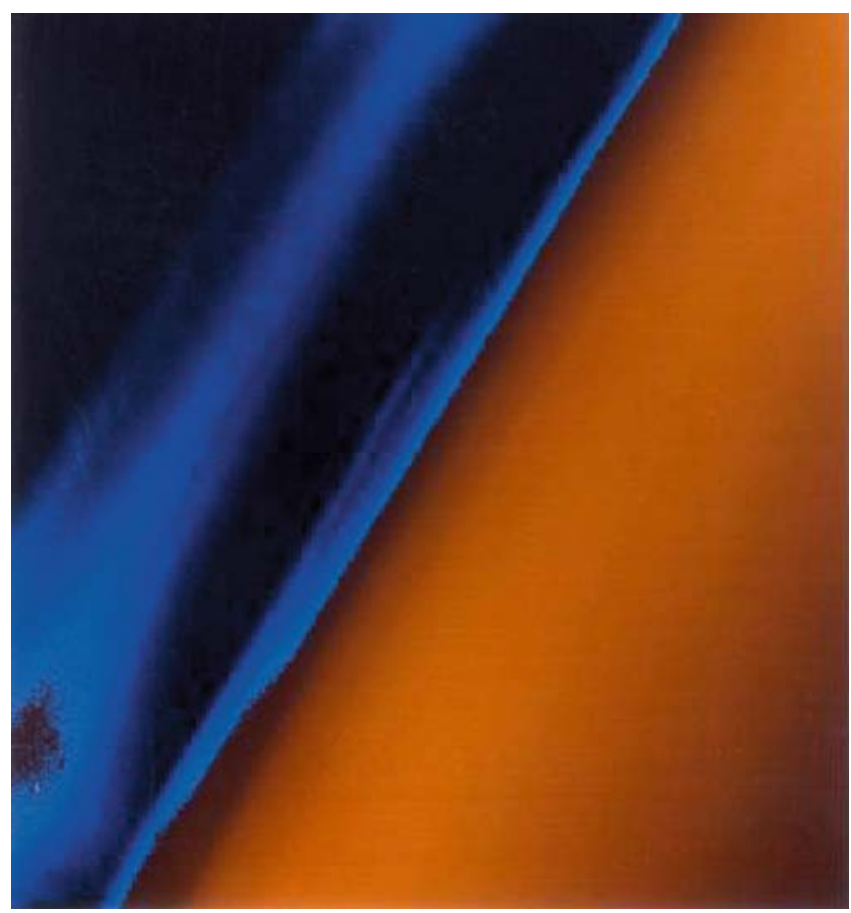

Fig. 13.15. Aerosol layers observed by Voyager 1 (courtesy of NASA)

the density profile of the atmosphere. Assuming hydrostatic equilibrium, the pressure profile can be deduced. Finally, the choice of a state equation allows to retrieve the ratio $T /\langle m\rangle$ as a function of altitude ( $T$ is the temperature and $\langle m\rangle$ the mean molecular mass).

Assuming that the atmosphere is entirely composed of molecular nitrogen, Lindal et al. (1983) determined the density, pressure and temperature profiles of Titan atmosphere. The temperature at the ground level is $94 \mathrm{~K}$ and the pressure 1495 mbar. The temperature decreases in the troposphere and the profile shows the presence of a tropopause with a temperature of $71.2 \mathrm{~K}$ at $40 \mathrm{~km}$ altitude ( $p \sim 120 \mathrm{mbar})$. The temperature then increases in the stratosphere to reach a temperature of $\sim 170 \mathrm{~K}$ at $200 \mathrm{~km}$ ( $p \sim 0.75 \mathrm{mbar}$ ) (see Fig. 13.18). The choice of molecular nitrogen as the major constituent seemed to be arbitrary since methane was the only abundant detected component. However, it has been a posteriori justified by the results obtained with the infrared and ultraviolet instruments.

The ultraviolet spectroscopic experiment (UVS for UltraViolet Spectrometer), which did measurements in the 60 to $160 \mathrm{~nm}$ wavelength range, observed in addition to Lyman $\alpha$ emission due to atomic hydrogen, an intense emission around 100nm (Broadfoot et al. 1981). This emission corresponds to the one 
obtained in laboratory experiments when $\mathrm{N}_{2}$ is excited by electronic impacts. The relative intensity of the bands allows to determine a column density of $\sim 10^{15} \mathrm{~cm}^{2}$. Besides, the ultraviolet occultation experiment which measured the attenuation of the solar flux through the atmosphere allowed to measure the atmospheric scale length ${ }^{10}$ at an altitude of $1265 \mathrm{~km}$ (altitude where the optical depth $^{11} \tau=1$ for wavelengths lower than $80 \mathrm{~nm}$ ), characteristic of nitrogen absorption: $H=85 \pm 10 \mathrm{~km}$ (Smith et al. 1982). Assuming that the atmosphere is entirely composed of nitrogen, this lead to a temperature $T=176 \pm 20 \mathrm{~K}$. Furthermore, knowing the absorption coefficient of $\mathrm{N}_{2}$ around $70 \mathrm{~nm}$, one can determine the local density: $\left[\mathrm{N}_{2}\right]=2.7 \pm 0.2 \times 10^{8} \mathrm{~cm}^{-3}$. A change in the scale length starts at an altitude of $1125 \mathrm{~km}$ and is attributed to methane. Smith et al. (1982) deduced from those measurements a mixing ratio $\left[\mathrm{CH}_{4}\right] /\left[\mathrm{N}_{2}\right]=0.08 \pm$ 0.03 (making the assumption of a constant temperature). They also determined a mixing ratio for acetylene of 1 to $2 \%$ above $825 \mathrm{~km}$ and less than $0.3 \%$ below $675 \mathrm{~km}$. This would imply a high sink for this compound in this region of the atmosphere. The data also showed highly absorbing layers around $390 \mathrm{~km}$ and $760 \mathrm{~km}$, with a $\sim 70 \mathrm{~km}$ thickness. The first layer seemed to be well correlated to the high altitude layer observed in the visible while the second one is not. Since in this region, $\mathrm{C}_{2} \mathrm{H}_{2}$ abundance decreases rapidly one can think that it could be efficiently photolysed in this region of the atmosphere, leading to the formation of higher weight compounds. This emission spectrum has lead to the determination of upper limits for the mixing ratio of $\mathrm{H}_{2}$, $\mathrm{Ar}$ and $\mathrm{Ne}$ in the high atmosphere: $\left[\mathrm{H}_{2}\right] /\left[\mathrm{N}_{2}\right] \leq 0.06,[\mathrm{Ar}] /\left[\mathrm{N}_{2}\right] \leq 0.06,[\mathrm{Ne}] /\left[\mathrm{N}_{2}\right] \leq 0.01$ and $[\mathrm{CO}] /\left[\mathrm{N}_{2}\right]$ $\leq 0.05$ (Strobel and Shemansky 1982). As a conclusion, the UV data showed that nitrogen is the major compound in Titan's atmosphere, so that it should be the place for an active photochemistry involving nitrogen and methane. The confirmation came from the analysis of the infrared spectroscopic measurements.

The infrared spectrometer (IRIS for Infrared Radiometer and Interferometer Spectrometer) onboard the Voyager spacecraft was a Fourier transform spectrometer (resolution $4.3 \mathrm{~cm}^{-1}$ ) covering the domain 180 to $2400 \mathrm{~cm}^{-1}$ (55 to $4.2 \mu \mathrm{m})$. The spectrometer of the Voyager 1 spacecraft took almost 3000 spectra during the Titan flyby and a wide latitudinal-longitudinal geographical region was explored. The first analysis of the spectra obtained at the equator confirmed the presence of methane $\left(\mathrm{CH}_{4}\right)$, ethane $\left(\mathrm{C}_{2} \mathrm{H}_{6}\right)$, acetylene $\left(\mathrm{C}_{2} \mathrm{H}_{2}\right)$ but also the presence of ethylene $\left(\mathrm{C}_{2} \mathrm{H}_{4}\right)$ and hydrogen cyanide (HCN) (Hanel et al. 1981). All these compounds are seen in emission showing that they are present in the relatively warm stratosphere of Titan. The study of Titan's north pole completes this list adding propane $\left(\mathrm{C}_{3} \mathrm{H}_{8}\right)$, methyl acetylene $\left(\mathrm{C}_{3} \mathrm{H}_{4}\right)$ (Maguire et al. 1981), diacetylene $\left(\mathrm{C}_{4} \mathrm{H}_{2}\right)$, cyanoacetylene $\left(\mathrm{HC}_{3} \mathrm{~N}\right)$, and cyanogen $\left(\mathrm{C}_{2} \mathrm{~N}_{2}\right)$

10 The atmospheric scale length is the distance over which the pressure is divided by a factor $e$ (2.718). It is proportional to the temperature, and inversely proportional to the mean molecular mass and gravity.

11 The optical depth is the quantity, which represents the opacity of a material. When it equals one, the light intensity is attenuated by a factor $e$. 


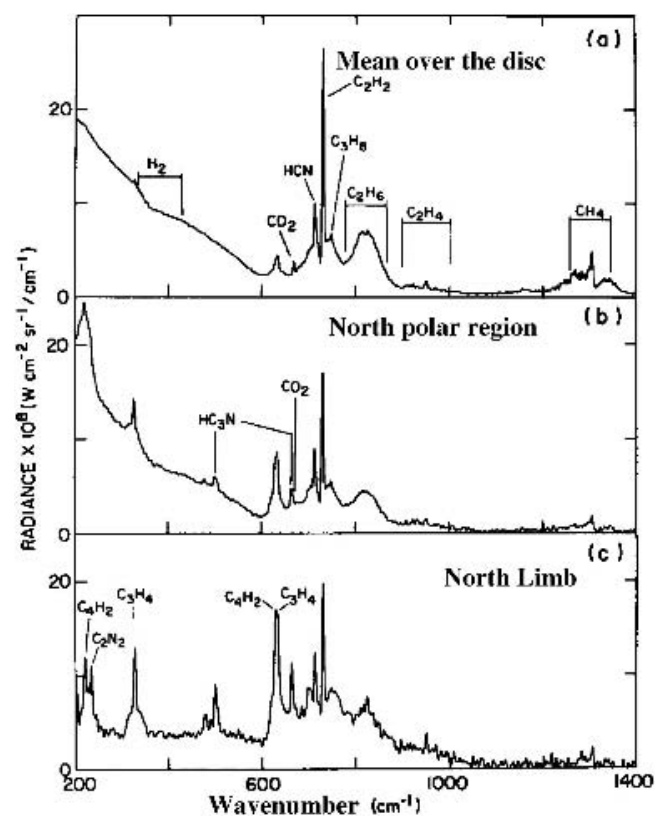

Fig. 13.16. Infrared spectra obtained at different latitudes (a and $\mathbf{b}$ ) and at the limb (c) by the IRIS experiment of the Voyager 1 mission. The main detected compounds are quoted (from Samuelson et al. 1983)

(Kunde et al. 1981). The presence of all those compounds in the stratosphere confirm the complex and active chemistry that is occurring in the $\mathrm{N}_{2} / \mathrm{CH}_{4}$ mixture which composes Titan's atmosphere.

A more careful study of the spectra obtained at different latitudes allowed Samuelson et al. (1983) to discover the presence of an oxygenated compound: $\mathrm{CO}_{2}$ (Fig. 13.16). In fact, even if its mole fraction is very low $\sim 1.4 \times 10^{-8}$, its distribution is uniform over Titan's disc, which is not the case for the other minor compounds. This first detection of oxygenated compound in Titan's atmosphere was later confirmed by the detection of CO in the near-infrared (Lutz et al. 1983) and millimeter (Marten et al. 1988) observations.

\subsubsection{Similarities and Differences Between Titan and the Earth}

The results obtained from the data accumulated by the Voyager missions have lead to a real breakthrough in our knowledge of Titan. One of the major results was the discovery of a dense atmosphere mainly composed of nitrogen in which the very active coupled chemistry of nitrogen and methane leads to the formation of numerous organic compounds. This makes Titan a unique object in the solar system since it the only one, outside the Earth, to have a dense atmosphere mainly made of nitrogen. Its slightly reductive atmosphere, in between the highly 


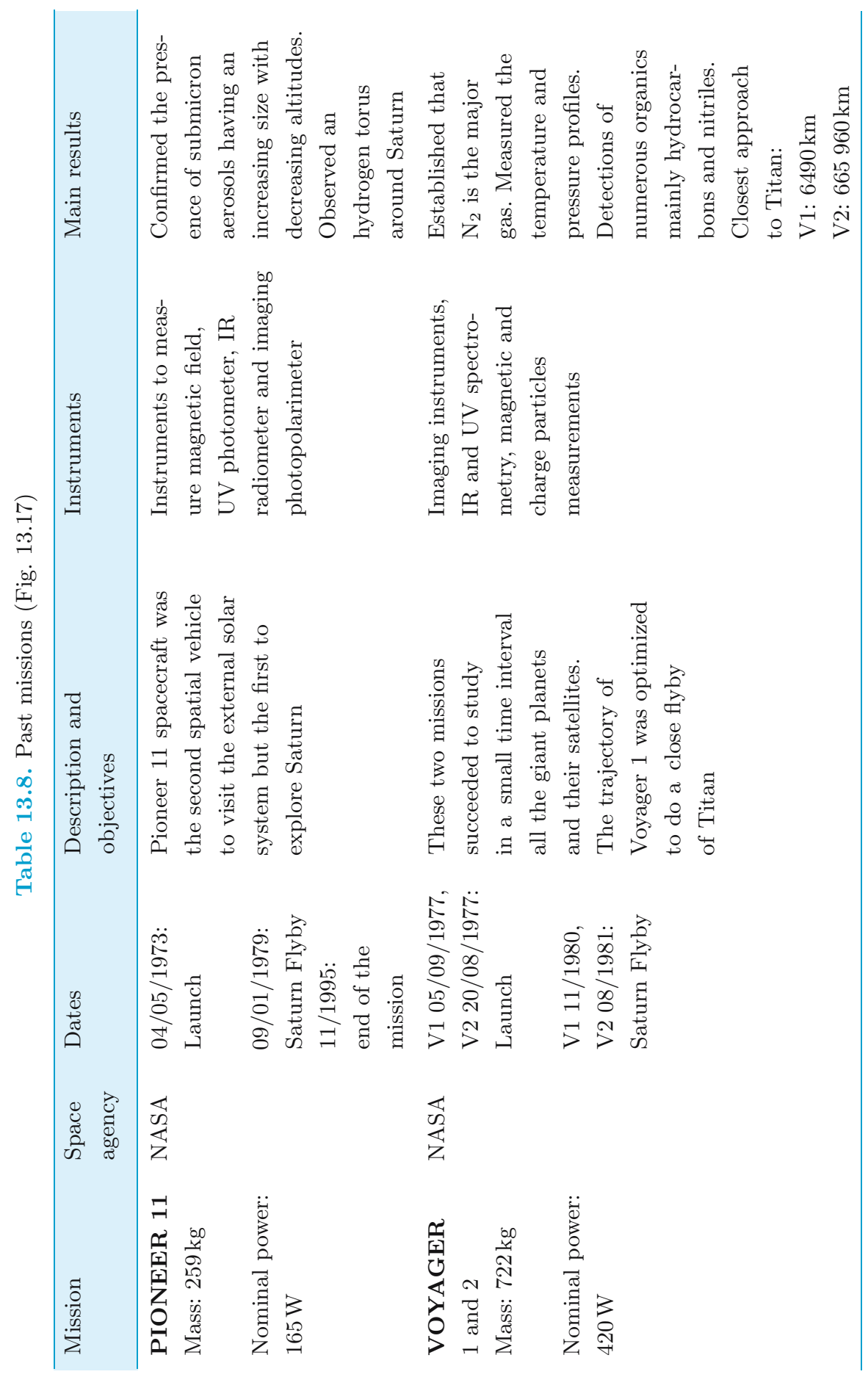




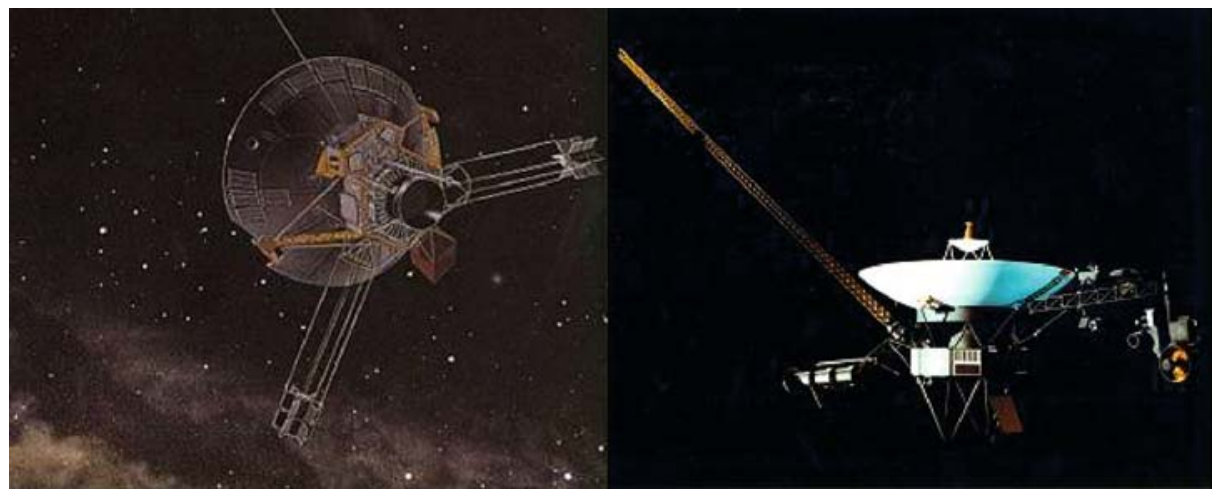

Fig. 13.17. Pioneer 11 and Voyager spacecrafts (courtesy of NASA)

reductive atmospheres of the giant planets and the highly oxidant atmospheres of the telluric planets, makes it a photochemical reactor which leads to the production of complex organic compounds. With this unique characteristic, Titan can be considered as a planetary scale laboratory for the study of the evolution of organic matter in abiotic conditions.

Titan's environment can help us to understand the primitive Earth and the chemical evolution that lead to the emergence of Life. The analogy between both objects is underlined by several common aspects. Both have a dense atmosphere mainly composed of nitrogen and in which the temperature profiles present similar structures. Hence, if $\mathrm{CO}_{2}$ is the major greenhouse gas in the terrestrial troposphere, in Titan, nitrogen, methane and hydrogen play this role through their pressure-induced absorptions. Warming in Titan's stratosphere is explained by ultraviolet absorption by small particles, which cannot emit efficiently in the infrared, where on Earth, ozone plays this role. The temperature in the terrestrial mesosphere is controlled by $\mathrm{CO}_{2}$ infrared re-emission where on Titan it is mainly HCN, which plays this cooling role. Finally, in the very high atmosphere of both objects the gas is heated by ionization. Therefore, the structures of both atmospheres are similar because they are controlled by similar physical processes. Only the chemical compounds implied in those processes changes. We can also add the possible presence of clouds in the low atmosphere of Titan due to methane condensation that could be similar to our water clouds on Earth. The presence on Titan of liquid reservoirs such as lakes or seas of methane and ethane has also been suggested.

Meanwhile, those similarities between Titan and the Earth should not mask the major differences. For example, while the surface pressures are approximately the same, the mean ground temperatures are very different: $-180^{\circ} \mathrm{C}$ on Titan compared to $20^{\circ} \mathrm{C}$ on Earth. This leads to a five times higher density on Titan than on the Earth at the ground level. Another difference appears if we compare the compositions of both atmospheres: nitrogen is the major compound for both of them but methane $\left(\mathrm{CH}_{4}\right)$ is second in Titan whereas nowadays on 


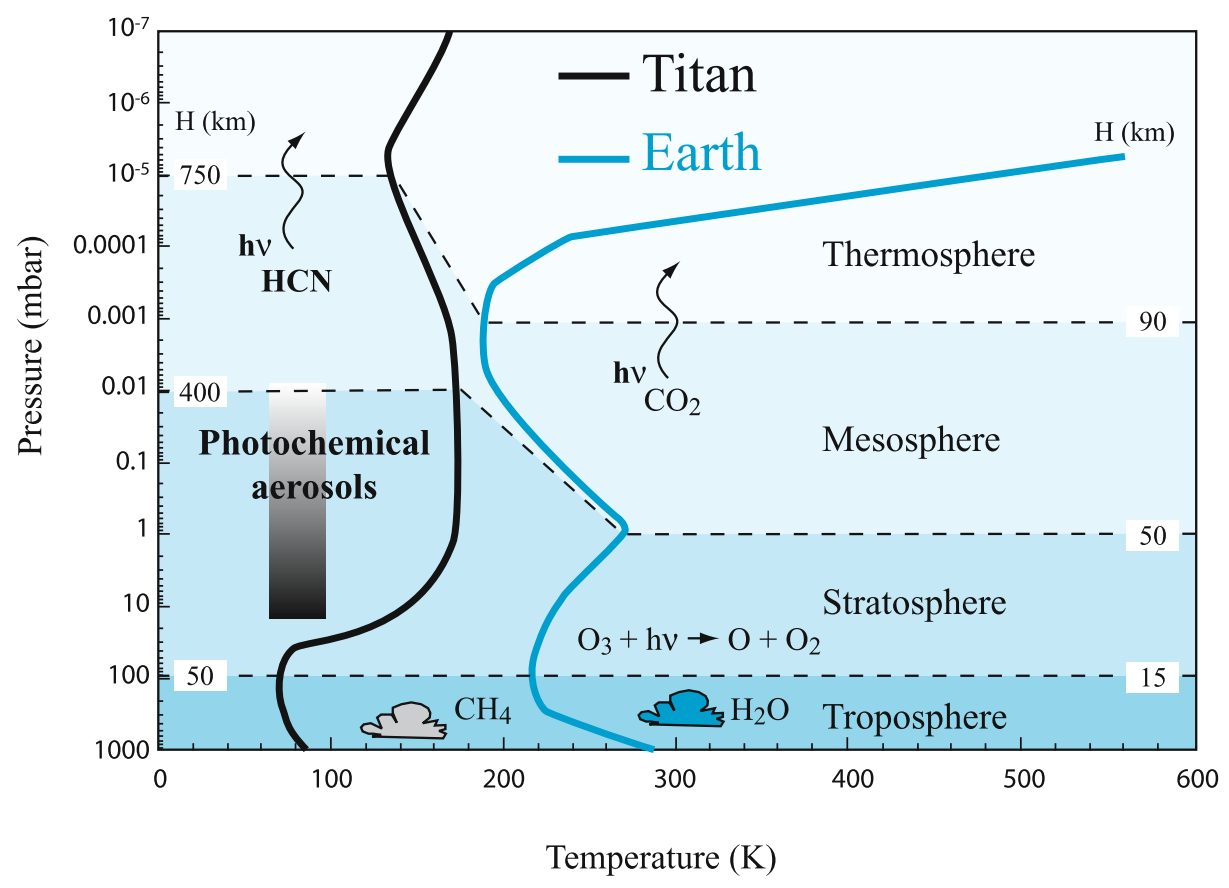

Fig. 13.18. Comparison between Earth's and Titan's atmospheric profiles

Earth it is molecular oxygen and water. Furthermore, carbon appears mostly on Earth in its oxidized state $\mathrm{CO}_{2}$. Water certainly played an important role in the appearance of life. Traces of water vapor have also been detected in the high atmosphere of Titan by Coustenis et al. (1998), using the ISO satellite (Infrared Space Observatory), but at the temperature of Titan's surface, this water is certainly solid. Nevertheless, Thompson and Sagan (1991) showed that meteoritic impacts on Titan could have lead to ejecta containing water liquefied under the shock. According to this model, large quantities of liquid water could have persisted on the surface for a total duration of several thousands years. However, would this be enough for an aqueous organic chemistry to develop and produce prebiotic molecules? Finally, the presence of an underground ocean, as for example in Europa, opens other exobiological perspectives (Fortes 2000), but as already mentioned the question of the transport between this ocean and the surface remains a major unsolved problem.

In Titan's atmosphere, a solid phase is also present as dust particles. By condensation at their surface volatiles can be carried to the surface and possibly into a liquid methane/ethane surface. Therefore, Titan contains all the elements of what we can call a "geofluid" (Raulin 1997). This underlines the importance of the study of this satellite for exobiology. Indeed, this is the planetary body that is the closest to what could have been the Earth before the appearance 
of life. Of course, the major difference with the primitive Earth is certainly the very low temperature of Titan, which implies the absence of liquid water. Nevertheless, the similitude between what could have been the primitive Earth and Titan, is one of the main reasons that motivated the launch of a probe called Huygens, which entered Titan's atmosphere in January 2005 to do in situ measurements.

Finally, one will note that even if in situ measurements bring data of fundamental importance on the composition, structure and dynamic of the atmosphere, they are done on a single trajectory of the space-time domain. Therefore, they have to be completed by teledetection measurements that allow to study phenomena at large scales with their time variations. This is done by the instruments onboard the Cassini spacecraft that has been put into orbit around Saturn in July 2004 for a minimum period of four years.

\subsubsection{Cassini-Huygens Mission}

The Cassini-Huygens space mission is a joint mission from ESA and NASA (Fig. 13.21). It is composed of an orbiter Cassini (NASA) and a descent probe Huygens (ESA). The whole set of instruments onboard is given in Table 13.9.

The main goal of the Huygens probe was to study the atmosphere and the surface of Titan. It has measured (in situ) the physical characteristics and chemical composition of the atmosphere and the surface. It was equipped with six main instruments. After being released by the orbiter Cassini, it entered Titan's atmosphere on January 14, 2005 and went through a first deceleration protected by its thermal shield. Its first small parachute opened around $180 \mathrm{~km}$ and caused the opening of a second much larger parachute (Lebreton and Matson 1997). As soon as the main parachute was inflated the shield dropped out around the altitude of $160 \mathrm{~km}$. Forty-two seconds later, the first measurements started. Because of the limited capacity of the batteries $(1.8 \mathrm{kWh})$, a quarter later when the probe was around $125 \mathrm{~km}$ altitude, the main parachute was replaced by a smaller one. With a maximum duration of 153 minutes predicted for the whole mission, the probe was supposed to do measurements on the surface for at least 3 minutes. Nevertheless, the reality exceeded all the predictions. After a 152-minute descent, the probe landed on Titan smoothly $(4.5 \mathrm{~m} / \mathrm{s}$ on humid sand like material) and continued to emit toward Cassini for more than an hour (the probe actually continued to emit for several hours but no relay could send back the signal to Earth).

One of the key objectives of the Huygens probe was to determine the chemical species present under gaseous, solid or liquid phase in the atmosphere and on the surface. However, further than simply obtaining quantities the main goal was to study the coupling between those different phases in order to try to understand the evolution that occurred on Titan since its formation. It is this approach that should allow to gain new elements to understand the prebiotic chemistry that could have occurred on Earth before the emergence of life. In 


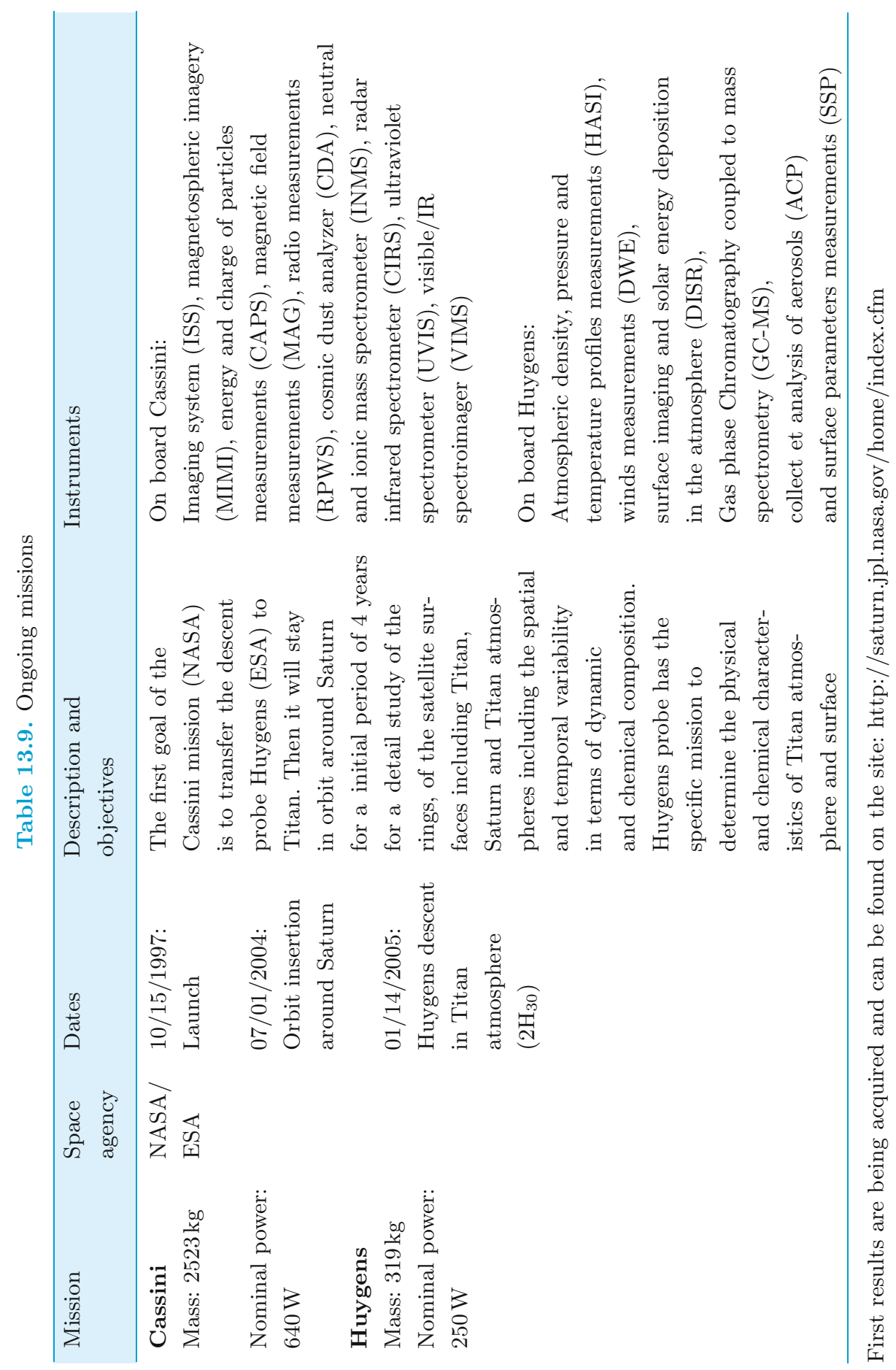


fact, if the "prebiotic chemist" is able to synthesize in the laboratory almost all biomacromolecules starting from hydrocarbons and nitriles observed on Titan, the question about what could happen on a planetary scale over geological time is still open. The questions that remain are (Raulin 1997):

- What complexity could achieve organic synthesis in a reduced atmosphere where liquid water is absent?

- What could have the importance of multiphasic processes?

- What is the influence of the physical conditions (temperature, energetic deposition and dynamics) on the chemistry?

The study of Titan (environment on which liquid water is absent, temperature very low, and energy sources diverse) can supply elements that answer those questions. The whole set of experiments on board the Huygens probe will contribute: the gas phase chromatograph coupled to a mass spectrometer (GCMS, Gas Chromatograph Mass Spectrometer), the collect and analysis of aerosols (ACP, Aerosol Collector Pyrolyser), the imager spectroradiometer (DISR, Descent Imager/Spectral Radiometer), the analysis of the surface (SSP, Surface Science Package) and the determination of the atmospheric structure (HASI, Huygens Atmospheric Structure Instrument).

\section{a) GCMS}

The instrument is composed of a chromatographic system made of three columns coupled to a mass spectrometer: one column was devoted to the measurement of permanent gases, one to light hydrocarbons (up to three carbons) and finally one is for heavier compounds up to eight nitrogen or carbons atoms (Niemann et al. 1997). The atmosphere has been analyzed during the entire descent by direct injection in the mass spectrometer (2-141 daltons). During the first half hour, a system was able to concentrate noble gases before their injection into the mass spectrometer. The chromatographic system itself was used on five different gaseous samples coming from five different altitudes. The first one was taken just after the start of the instruments around $150 \mathrm{~km}$. Two samples were then taken above $60 \mathrm{~km}$ altitude where the concentration of complex compounds was expected to be the highest. The next analysis was made on a sample obtained by the pyrolysis of the aerosols collected by ACP (see below). At the minimum temperature of the tropopause, the analysis was devoted to the separation of $\mathrm{N}_{2}$ and CO. Finally, since the descent time was nominal a last sample has been injected before impact. Moreover, since by chance the injector was correctly positioned, measurements of the surface composition have been done. The sensibility of the instrument was supposed to be very high of about ten parts per billion for most compounds. All the instrumentation worked correctly except the ion source attached to the separation of $\mathrm{N}_{2}$ and CO. The first analysis of the measurements has shown an increase of the methane concentration occurring just after impact. 


\section{b) $\mathrm{ACP}$}

Two regions of the atmosphere were sounded by this instrument: the first one between 135 and $32 \mathrm{~km}$ and the second one between 22 and $17 \mathrm{~km}$ (Israel et al. 1997). The aerosols were collected on a filter, which was placed a few millimeters outside the probe, and then taken back inside the pyrolysis oven. The oven allowed a warming of the samples up to $600^{\circ} \mathrm{C}$. The following sequence was planned: (1) collect during the first hour of the descent then warming at ambient temperature, $250^{\circ} \mathrm{C}$ and $600^{\circ} \mathrm{C}$, then transfer to GCMS; and (2) collect a second sample above possible clouds then warm at the three temperatures and transfer to the GCMS. The main goal of those measurements was to determine the composition of the condensation nuclei by comparing the measurements obtained in Titan atmosphere with analogues produced in laboratory simulations. The relative composition of the condensates present on the aerosol surface should be obtained. Finally, the possible abundance of adsorbed volatile like $\mathrm{CO}$ in the aerosols might be determined. The system seems to have worked nominally.

\section{c) DISR}

During the whole descent, optical instrumentation of DISR $(0.35-1.7 \mu \mathrm{m}$ domain) measured the upwelling and downwelling solar flux in order to determine the net flux (Tomasko et al. 1997). The difference between net fluxes taken at two different altitudes allows to determine the energy deposited. This enables the understanding of the thermal balance of the atmosphere. Measuring the diffusion properties, for two colors and two polarizations, together with the extinction of the aerosols leads to the determination of the refractive index. Since this quantity is characteristic of a given material, it should be possible to constrain the chemical composition of Titan's aerosols. This optical instrumentation should allow to obtain the methane mixing ratio as a function of altitude by measuring its absorption and should also help to constraint the nature of the surface by determining its reflectivity. The measurements continued even in the last minutes of the descent when the probe was getting closer to the surface (where the atmosphere became more and more opaque) thanks to a $20 \mathrm{~W}$ lamp attached to the instrument. DISR also includes two cameras that obtained images of the surface with a resolution going from a few hundred meters (like the orbiter) at the beginning of the descent to a few tenths of a centimeter when approaching the surface. The first analysis of those images showed the presence of geological structures characteristic of precipitation, erosion and fluvial activity similar to what is observed on Earth (Fig. 13.19). The surface is relatively flat and on the impact site, the presence of ice blocks of a few teens of centimeters can be observed (Fig. 13.20). The surface seems to be dry around the impact site but methane rainfall might have occurred recently. 


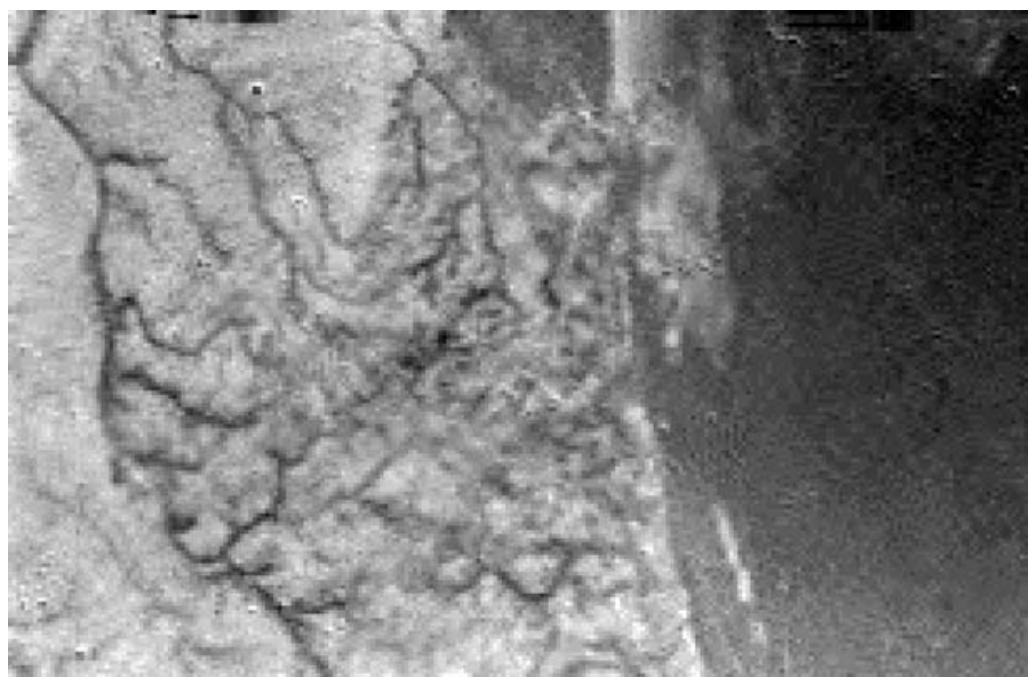

Fig. 13.19. This image was obtained by DISR at $16.2 \mathrm{~km}$ above the Titan surface. The resolution is 40 meters per pixel. The presence of a fluvial network and a coastline can be observed (courtesy of ESA/NASA/JPL/University of Arizona)

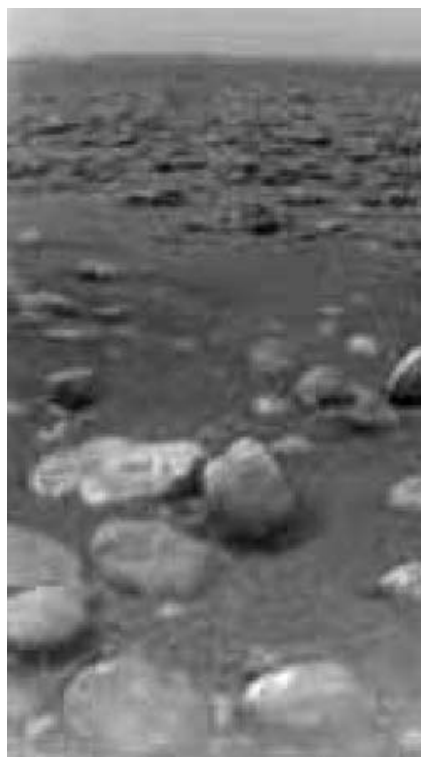

Fig. 13.20. This image was obtained after the Huygens probe landed on the Titan surface. The surface is relatively flat and several ice blocks can be identified. The typical size of those blocks is fifteen centimeters (courtesy of ESA/NASA/JPL/University of Arizona) 


\section{d) HASI}

The first goal of this instrumentation was to determine the density, pressure and temperature profiles all along the descent (Fulchignoni et al. 1997). However, this experiment also included the possibility to measure the electric field, the electric conductivity and even the sound waves through a microphone. Thus, it should be possible to determine the electronic density in the atmosphere but also deduce the presence of lightning storms if those are present around the descent trajectory. The conductivity of the surface could also be measured after landing which can help to constrain the nature of the surface. The first analysis showed that the measured pressure and temperature vertical profiles are very consistent with the one obtained by Voyager and no lightning was observed.

\section{e) SSP}

This experiment was composed of nine different instruments (Zarnecki et al. 1997). The set of instruments has been made to give information on the surface whether solid or liquid. If the surface had been liquid, the thermal conductivity, the density, the sound speed, the refracting index, and the permittivity could have been determined. Nevertheless, it was the accelerometer and the penetrometer that gave the interesting information since the surface was solid: granularity, penetration resistance and cohesion could be determined. An acoustic sounding device will give an idea of the topography of the surface. The sound speed, the temperature or the permittivity of the atmosphere were also determined during the descent

The instruments of the orbiter will also contribute. In fact, they allow a global view, which is essential to interpret the probe data. The two major instruments that supply data concerning Titan's atmospheric composition are:

\section{f) CIRS}

This instrument is an infrared Fourier transform spectrometer, which acquires data in the range of 10 to $1400 \mathrm{~cm}^{-1}(1 \mathrm{~mm}$ to $7 \mu \mathrm{m})$ (Flasar et al. 2004). This improved version of the IRIS spectrometer that was on the Voyager spacecrafts (extension of the wavelength domain, of the resolution and of the sensitivity) allows a mapping in three dimensions not only the chemical composition of Titan's atmosphere but also its temperature.

\section{g) UVIS}

This ultraviolet spectrometer is composed of two channels that study the radiations that are coming from Titan between 56 and 190 nm (Esposito et al. 2003). It also obtains limb data from solar or stellar occultation. This should permit the determination of the vertical profile of numerous species in the high atmosphere where a large amount of the energy is deposited. 


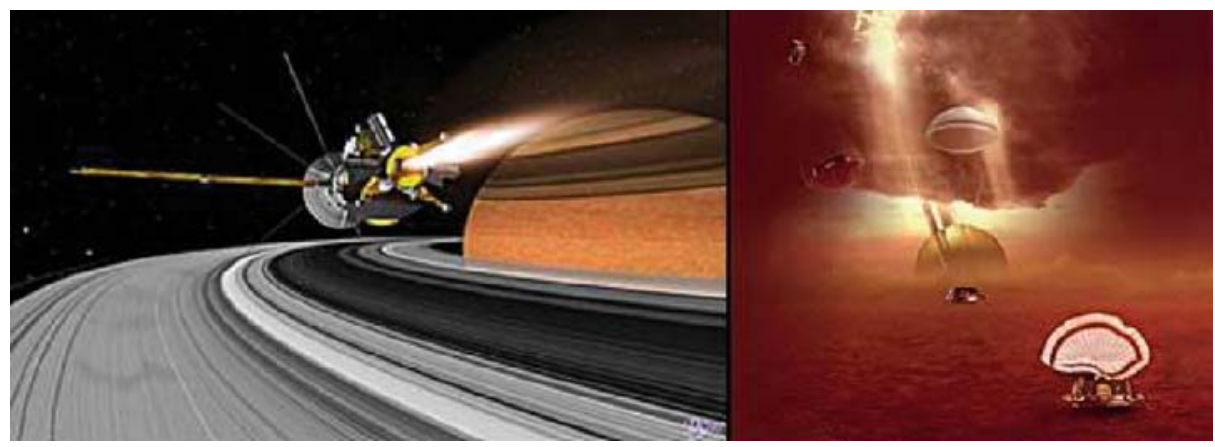

Fig. 13.21. Cassini-Huygens spacecraft arriving in the Saturn system and Huygens probe descent inside Titan's atmosphere

The results of this space mission will certainly lead to a large breakthrough in our understanding of organic processes that could lead to the emergence of life on a planet having a reductive atmosphere and liquid water. However, even if the Cassini-Huygens mission will bring a very large amount of data it will certainly not answer all the questions concerning the complexity of Titan. Especially, since the descent was over one trajectory, it will not be possible to document the great complexity that already appears either on surface images or as a northsouth asymmetry. In the future, it is possible and desirable that a new mission could be sent but this time with a mobile laboratory that could study different regions of Titan surface and different altitudes in the atmosphere (Lorentz 2001).

\subsection{Mars Exploration}

\subsubsection{Mars Before Space Missions}

Planet Mars, known since the prehistoric times (Egyptians were already talking about it in $4000 \mathrm{BC}$ ) has always attracted human beings. This is certainly partly due to its special trajectory in the sky, its aspect (its apparent diameter can vary by a factor greater than five), and its reddish color. On November 28, 1659, Huygens observed for the first time structures on the disk of Mars that changed with time (Fig. 13.22). From his observations, he deduced a rotational period of about 24 hours as on Earth. This gave rise to several phantasms that tended to assimilate the Martian world to the terrestrial one.

The observations made in the following centuries amplified those phantasms. Seas and "continents" appeared at the surface of the red planet. At the end of the nineteenth century, Lowell imagined that a channel network irrigates the surface of Mars to supply its inhabitants. It was necessary to wait for the beginning of space exploration to definitively drop the idea that a life similar to the one on Earth could be present. 

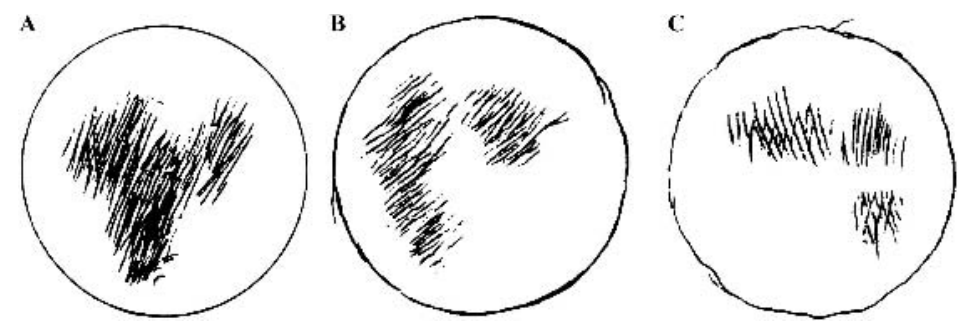

Fig. 13.22. Three drawings made by Huygens in 1659. The first one was drawn on November 28 at $7 \mathrm{pm}(\mathbf{A})$, the second was done the same day but at 9:30 pm. (B). The last one was made three days later December 1 at 6:30 pm (C)

Another hot topic was the one of the nature of the polar caps and the presence of water. Polar caps that were observed for the first time by Cassini have been followed through the centuries and the observer had noted their appearance and disappearance with seasons. They were first supposed to be made of water by analogy with the Earth but also because the measured temperature was not low enough for carbon dioxide to be present in a condensed phase (Hess 1948). According to this theory, water should have been observed in the atmosphere. However, it was necessary to wait for the observations of Spinrad et al. (1963) to detect trace amount of water vapor, about 10 precipitable microns. Finally, the first space missions were needed to have a better idea of the water cycle on Mars.

The nature of the Martian atmosphere itself (pressure, composition, temperature) has been the subject of many discussions. In October 1947, Kuiper observed Mars in the wavelength region around $1.6 \mu \mathrm{m}$. Comparing this spectra with the one obtained from the moon that contains telluric carbon dioxide absorption lines, he showed that the absorption was stronger in Martian spectra (Kuiper 1952). Nevertheless, he could not deduce from his data the abundance of $\mathrm{CO}_{2}$ because the bands were saturated. It was necessary to wait for the very high-resolution spectra obtained by Kaplan et al. (1964), in the $870 \mathrm{~nm}$ domain were the bands are optically thin, to measure the abundance of $\mathrm{CO}_{2}$ at the surface: 4 mbar. The total surface pressure was still unknown at that time. Nevertheless, using observations around $2 \mu \mathrm{m}$, Kuiper (1963) and Sinton (1963) deduced that the total pressure was between 10 and 40 mbar. Therefore, for the first time, carbon dioxide appears as the main constituent of the Martian atmosphere.

\subsubsection{The Beginning of Martian Exploration}

Only eleven of the thirty or so missions sent to Mars effectively reached their goals. The lost missions have been reported in Table 13.12. As can be seen there are various reasons of failure, explosion at ignition, communication lost during Mars transfer or, more recently, communication lost during landing. 
If the Soviets were the first to send a spacecraft to Mars, it is the US Mariner 4, launched November 28, 1964, which was the first to reach Mars on July 14, 1965. Thanks to its onboard camera, it took the first pictures of the Martian surface with a resolution of $4 \mathrm{~km}$. The ground appears riddled with craters, which lead to compare it with the moon, and seemed to indicate that the surface was as old as the planet itself (Chapman et al. 1969). But, only $1 \%$ of the surface had been pictured. The greatest breakthrough was done thanks to the radio occultation experiment that allowed for the first time to determine the surface pressure and the temperature. The pressure was of about 5 mbar (Kliore et al. 1965). Those measurements confirmed that carbon dioxide is the main element of a thin atmosphere. The next mission burst to Mars with Mariner 6 and 7 in 1969, followed by Mariner 9 in 1971 and finally the Viking probes in the mid 1970s, allowed to reach a more and more precise idea of the Martian environment using heavier and heavier equipment including more and more advanced technology (see Table 13.10).

Mariner 6 and 7 probes were equipped not only with a camera able to obtain images of $10 \%$ of the surface, but also an infrared spectrometer, an infrared radiometer and an ultraviolet spectrometer. The latest identified atomic hydrogen, atomic oxygen and carbon monoxide in the high atmosphere (Thomas 1971). The infrared measurements allowed to identify carbonic ice as the major component of the south pole (Herr and Pimentale 1969). The radiometer measured a temperature at the south pole of $148 \mathrm{~K}$, which led to confirm that it was composed of carbonic ice (Neugebauer et al. 1971). In fact, vapor pressure of $\mathrm{CO}_{2}$ at this temperature is about 6 mbar, which is compatible with the measurements of Kliore et al. (1971). In the same conditions, the vapor pressure of water is only $10 \times 10^{8}$ mbar. Finally, thanks to the UV spectrometer, ozone was also discovered.

The Mariner 9 interplanetary probe stayed in orbit for a period of almost one terrestrial year. This allowed the cartography of almost all the Martian surface, the study of the atmospheric physicochemistry and its temporal variations over a period ranging from one day to one month. Imagery experiment showed an unsuspected diversity of the surface with the presence of volcanoes among which the highest, Olympus Mons, reaches $26 \mathrm{~km}$ and canyons deeper than $6 \mathrm{~km}$ like Valles Marineris. Valleys, which could have been formed by an erosion process, were also observed. Furthermore, water vapor was detected for the first time in Martian orbit by the infrared Fourier transform spectrometer IRIS, (Hanel et al. 1972) during the north summer. The condensation of this water vapor in the form of crystals was also observed (Curan et al. 1973). All those results pushed forward for an in situ mission able to bring into light the possible traces of biological activity on Mars.

The Viking project included two vehicles, Viking 1 and Viking 2, each one composed of an orbiter and a probe able to land on the Martian surface after a site selection. The main objectives of the Viking missions were the study of the Martian surface with high-resolution imagery, the characterization of the 


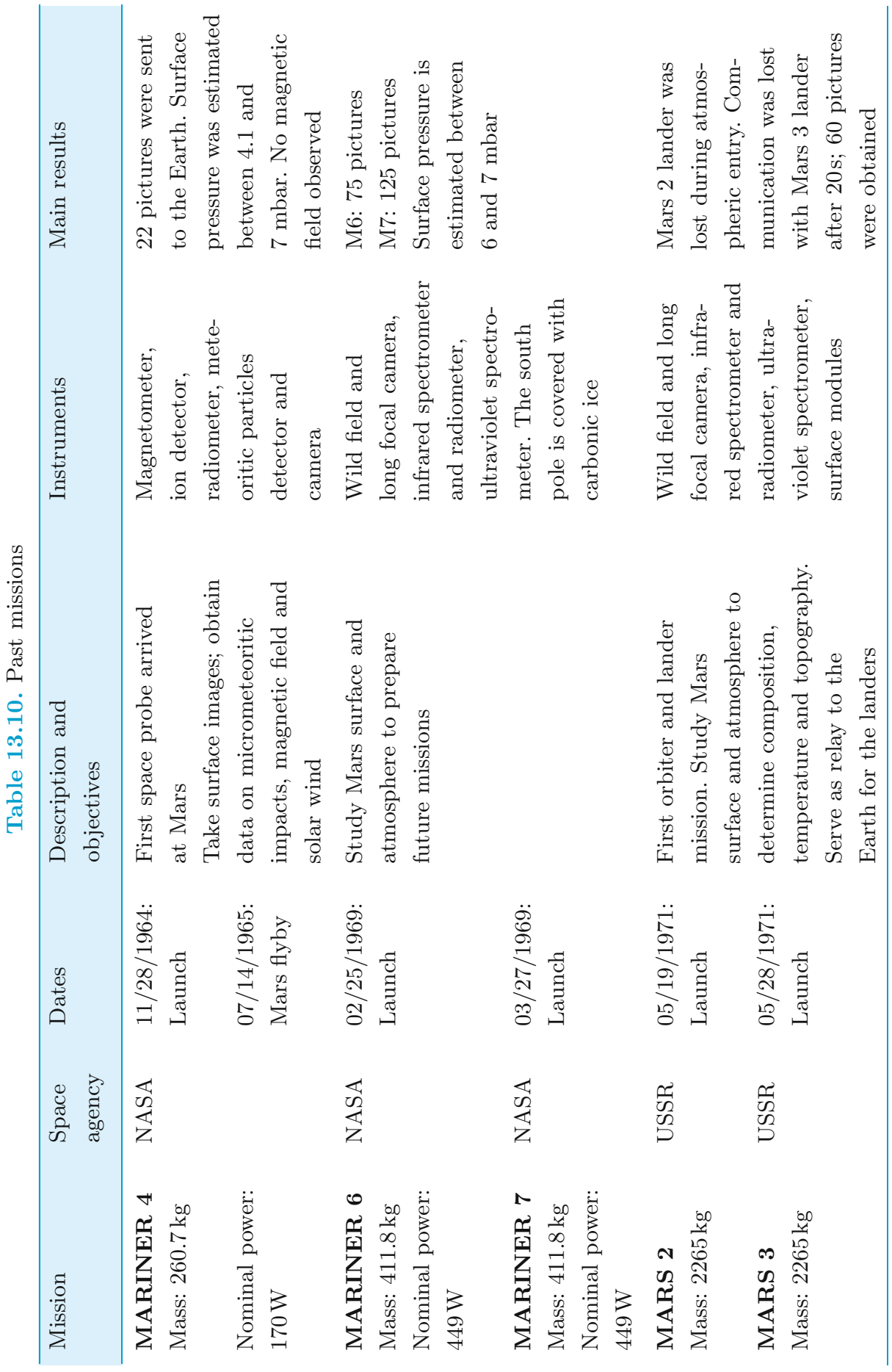




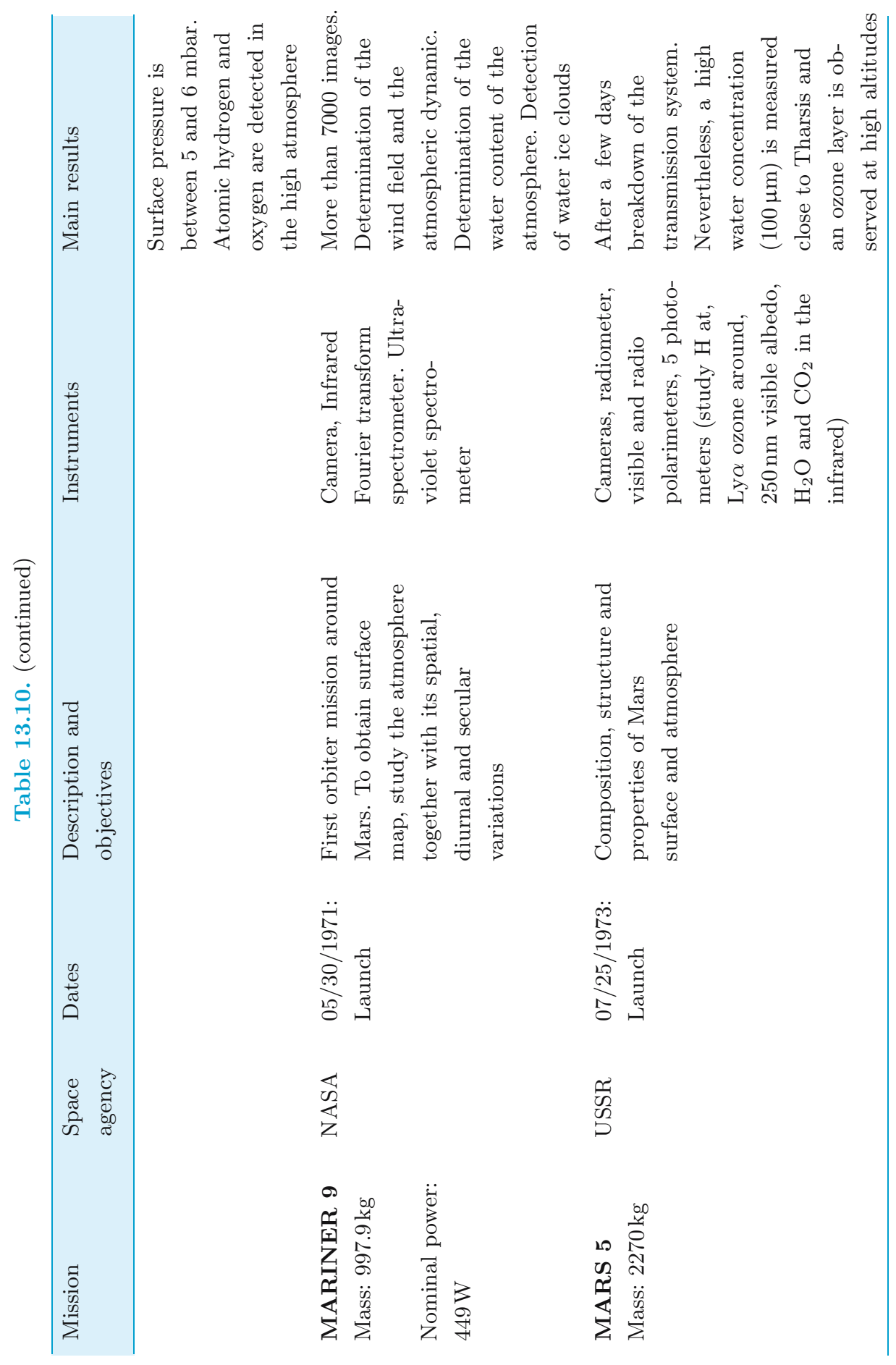




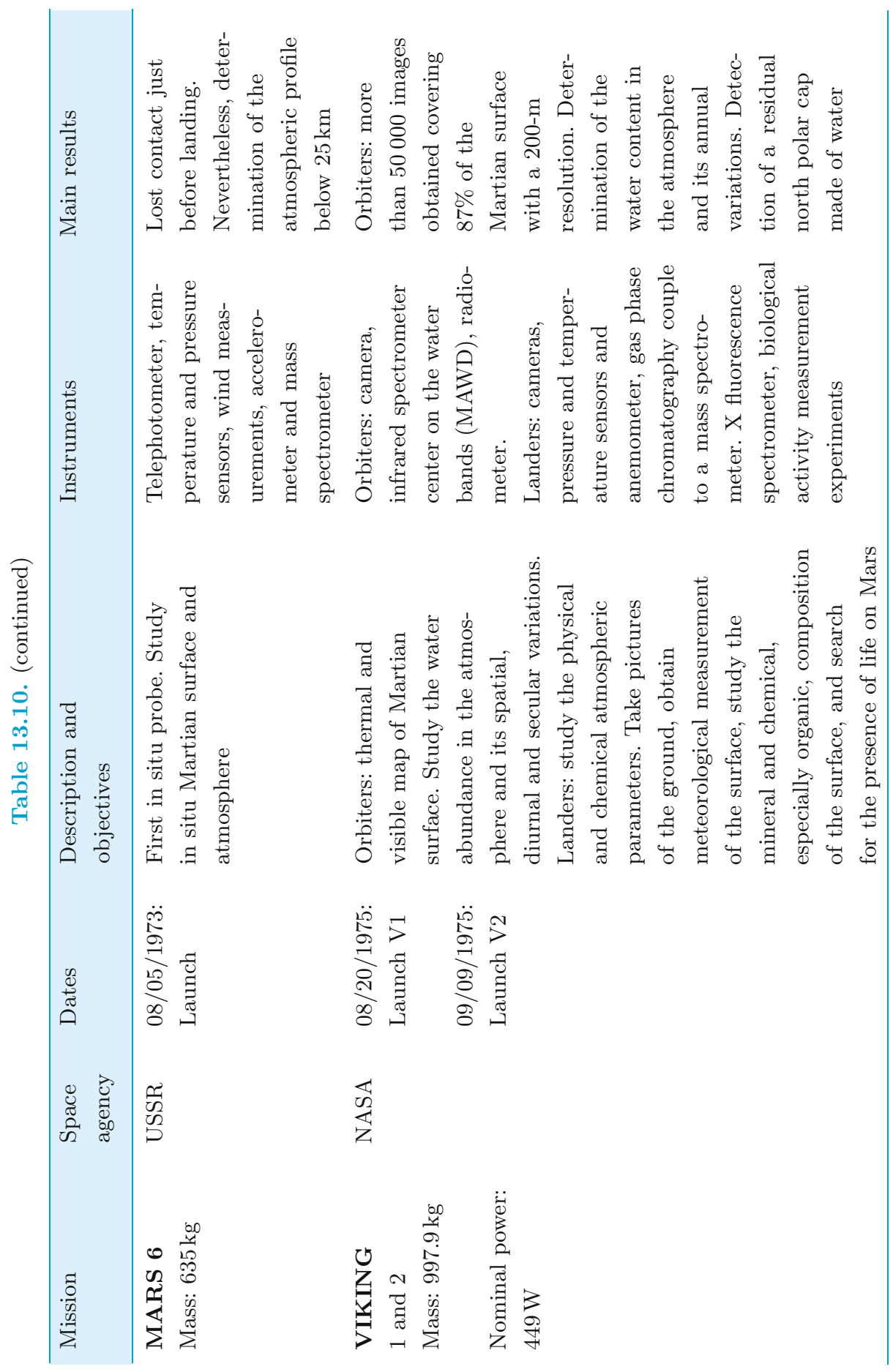




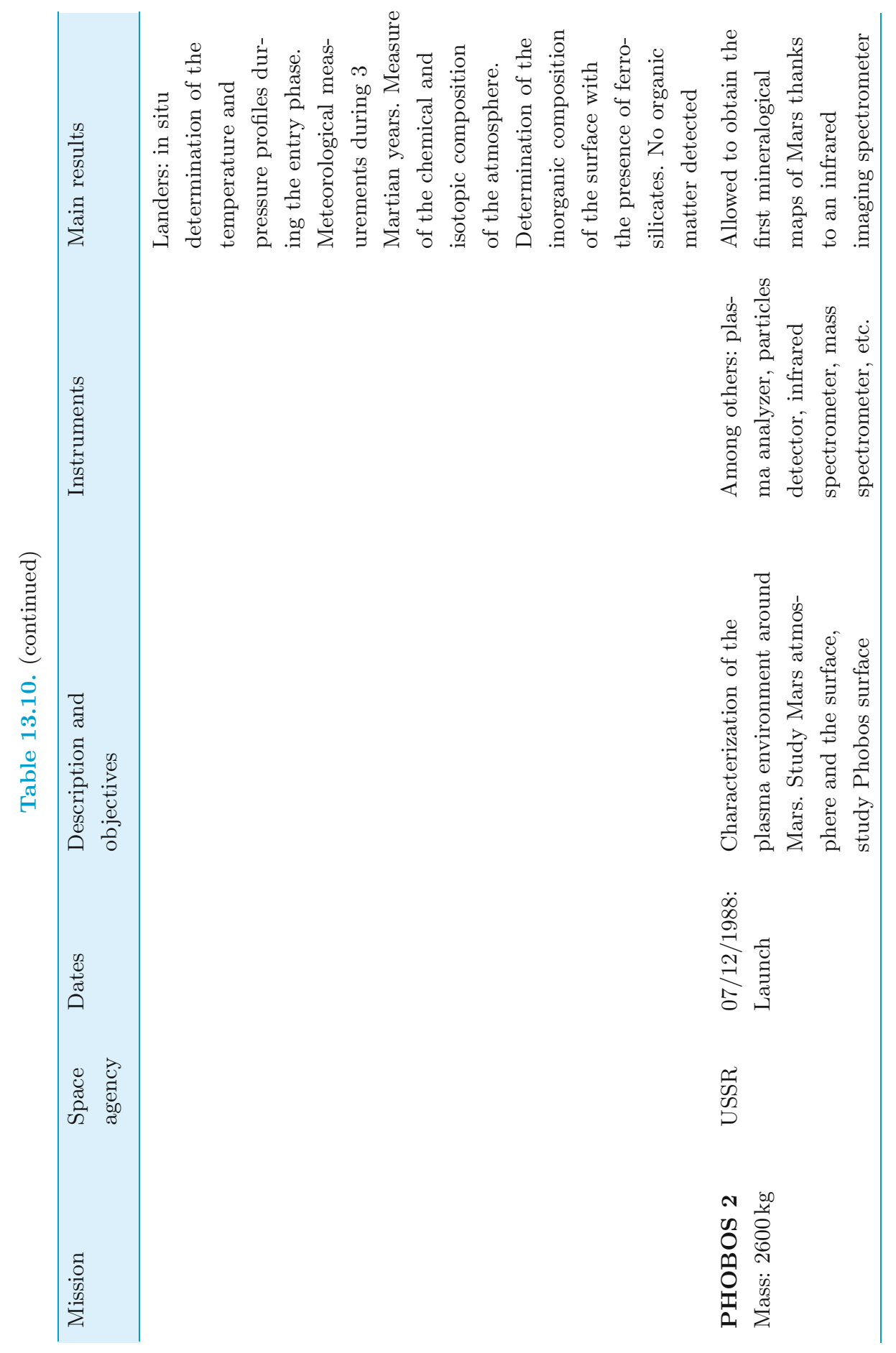




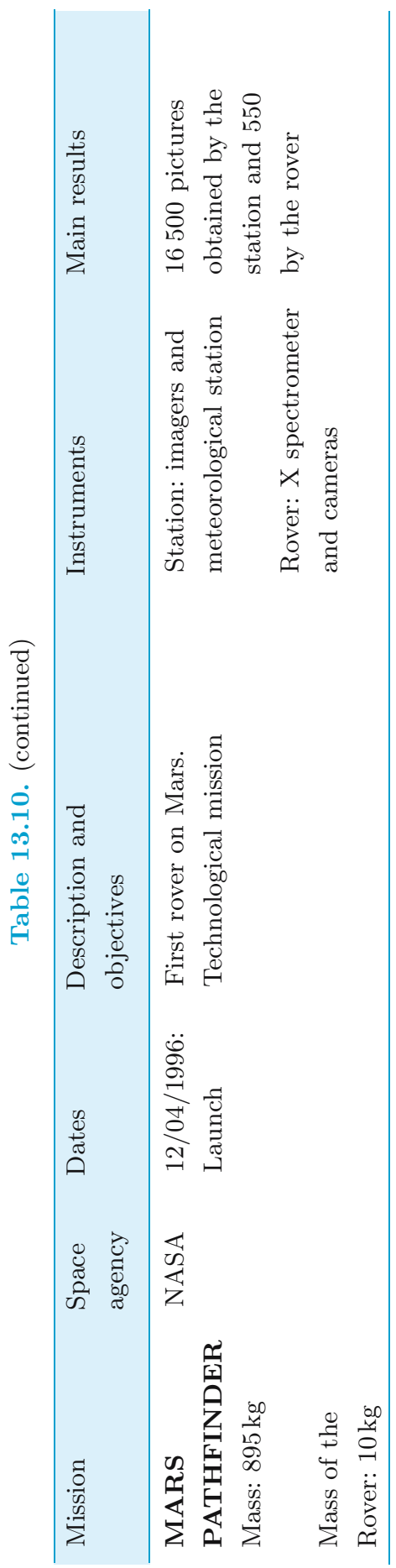


structure and the composition of the atmosphere and the surface and the search for possible forms of life.

The orbiters mapped the entire surface of Mars with a 200-meter resolution. In addition to the recurrent dust storms, they observed the variations of the surface pressure all along the Martian year. This evidenced the presence of a $\mathrm{CO}_{2}$ cycle, related to the decrease and the growth of the polar caps. The infrared spectrometer allowed to determine the water vapor content in the Martian atmosphere (MAWD, Mars Atmospheric Water Detectors) and to follow the atmospheric humidity global variations during the seasons due to the seasonal cycle of the water transport between the two poles. Finally, the coupling with the radiometer (IRTM, Infrared Thermal Mappers) results permitted the demonstration that if the south pole was mainly composed of $\mathrm{CO}_{2}$ ices, the residual north polar cap visible during the summer was mainly composed of water ice.

The Viking 1 lander made continuous measurements in the region of Chryse Planitia on the Martian surface during six terrestrial years whereas the Viking 2 lander operated during two Martian years in Utopia Planitia (Fig. 13.23). Each probe was carrying not only a set of experiment to detect possible life forms on the Martian surface but also instruments to study the chemical, magnetic, apparent and physical properties of the surface and the atmosphere, and to achieve meteorological and seismic observations.

A small meteorological station supported by a one-meter mast was composed of three thermal sensors distributed along the height, a pressure gauge and an anemometer allowing the measure of both wind speed and direction. Those mea-

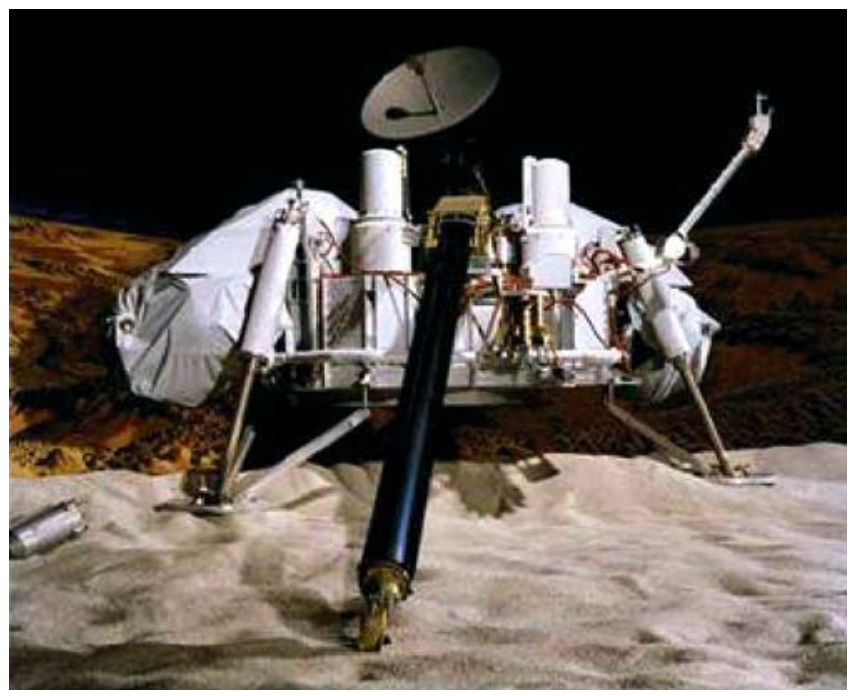

Fig. 13.23. One of the Viking landers with its mechanical arm deployed (photo courtesy of NASA/JPL) 
surements acquired during more than three Martian years constituted a unique database for the study of Mars climate.

Inorganic chemical analysis of the surface were made by $\mathrm{X}$ fluorescence (XRFS). The obtained results were disconcerting. If Mars seemed to be constituted mainly of silicon and oxygen like the Earth, iron instead of aluminum was the next most abundant element.

Each of the Viking landers was also equipped with a gas chromatograph coupled with a mass spectrometer (CPG-SM). They were designed to determine the molecular and isotopic atmospheric composition and to try to detect organic matter in the ground. The atmosphere could also be directly injected in the mass spectrometer. This led to the determination of the isotopic abundance of five constituents. Solid samples were first pyrolysed at $500^{\circ} \mathrm{C}$ and then the gaseous residues transferred to the chromatograph. No organic compounds were identified down to the threshold of one part per billion (Biemann et al. 1977). This result was surprising since even if no endogen sources were present, the exogenous sources should have enriched the Martian soil in organic compounds. One explanation is that organic matter might have been destroyed by the combined action of radiation sources and oxidation.

The exobiological experiment, onboard the Viking landers (Fig. 13.23), was composed of three distinct experiments (Klein et al. 1972). The first one was the "pyrolytic release". This experiment planned to detect carbonic gas assimilation $\left(\mathrm{CO}_{2}\right)$ either by photosynthetic mechanisms or in the dark. Martian samples were injected into a mixture of ${ }^{12} \mathrm{CO}_{2}$ and ${ }^{14} \mathrm{CO}$ (with a ratio of 95:5). During a five day period, the sample could be illuminated by a Xenon lamp simulating the solar flux but filtered below $320 \mathrm{~nm}$ to suppress UV. After this period, the residual atmosphere was evacuated and the sample pyrolysed in an oven at $625^{\circ} \mathrm{C}$. The organic matter was then extracted and the amount of ${ }^{14} \mathrm{C}$ determined. The procedure could be reproduced with water or in the dark. The results were difficult to interpret. First, experiments could not be reproduced: if the first experiment gave a positive result, the following one was negative. Furthermore, even if the sample was heated to high temperatures $\left(175^{\circ} \mathrm{C}\right)$ the results would stay positive. The best explanation is that the positive experiment could result from a chemical compound (for example, the formation of a polymer like carbon suboxide $\left(\mathrm{C}_{3} \mathrm{O}_{2}\right)$ ), or from a physical process like the adsorption of $\mathrm{CO}_{2}$ in the ground (Horowitz et al. 1977). The second experiment was the "gas Exchange". This experiment was made to detect different gases that living organisms could reject after ingestion of nutriments. A nutriment solution was added to surface samples in contact with the Martian atmosphere. For the calibration, part of the sample was heated for sterilization. The determination of the gas composition present over the sample was done through GC-MS analysis. Here again, results were astonishing as they showed a high oxygen degassing and a low release of $\mathrm{CO}_{2}$ and nitrogen. The release of oxygen was very quick and a second introduction of nutriment did not liberate more oxygen. Furthermore, the degassing occurred even in the dark or with the sterilized sample. Those re- 
sults could be explained if one supposes that Martian soil contains very reactive oxidant molecules like superoxides (Oyama and Berdahl 1977). Finally, the third experiment was the "labeled release". This experiment is similar to the previous one except that the carbon atoms of each compound inside the nutriment were radioactive. One detector could identify the outgassing radioactive $\mathrm{CO}_{2}$. A high outgassing of $\mathrm{CO}_{2}$ was observed after the injection of nutriment. This release was not observed with the sterilized samples. So, one could conclude the presence of biological activity from this experiment only. Nevertheless, this last result can also be explained by non-biological processes. For example, the action of hydrogen peroxide $\left(\mathrm{H}_{2} \mathrm{O}_{2}\right)$ could explain the release of carbon dioxide from the oxidation of the organic matter present in the nutriment (Huguenin et al. 1979).

The results of those experiments built to detect biological activity are still today subject to controversy because one can hardly deny the positive results of the label release experiment (Levin et al. 1981). Nevertheless, if microorganisms were really present inside the samples, organic matter should have been detected by the GC-MS experiment (Klein and Harold 1978). Consequently, most scientists agree to say that life is absent from the surface of Mars. Nevertheless, the question, if there is life on Mars, is still an open issue since some niches could exist in the subsurface.

\subsubsection{Current Space Missions}

Space missions currently in activity around Mars are resumed in Table 13.11. After the success of the 1970s and notably the Viking probe experiences, a long inactivity of almost twenty years occurred for the Martian program. In 1988, the Russian Space Agency put into orbit around Mars its first probe: Phobos 2. However, one had to wait until the end of the 1990s to see NASA land on Mars its first rover: Pathfinder. The mission is a true technological achievement and opens the way for a new Martian program. The missions that have followed had as a common objective to map the Martian surface and atmosphere to try to answer the question: when has there been water on Mars, in what state and where is it today? In fact, one of the essential needs for life seems to be liquid water. Consequently, we hope that following the water on Mars would lead us to the sources of life.

Within this objective, Mars Global Surveyor satellite brought its piece of the puzzle. The infrared imaging spectrometer allowed mapping the minerals present on the surface of Mars. Chistensen et al. (2000) identified the presence of hematite concentrated in a unique region: Meridiani Planum (Fig. 13.24). The presence of still water in the past could have allowed the precipitation of this iron oxide (Chistensen et al. 2004).

This observation led to the selection of this site for the landing of one of the Mars Explorating Rovers mission, MER B alias Opportunity. The other rover (MER A alias Spirit) landed in the Gusev Crater (Fig. 13.25). MER B confirmed 


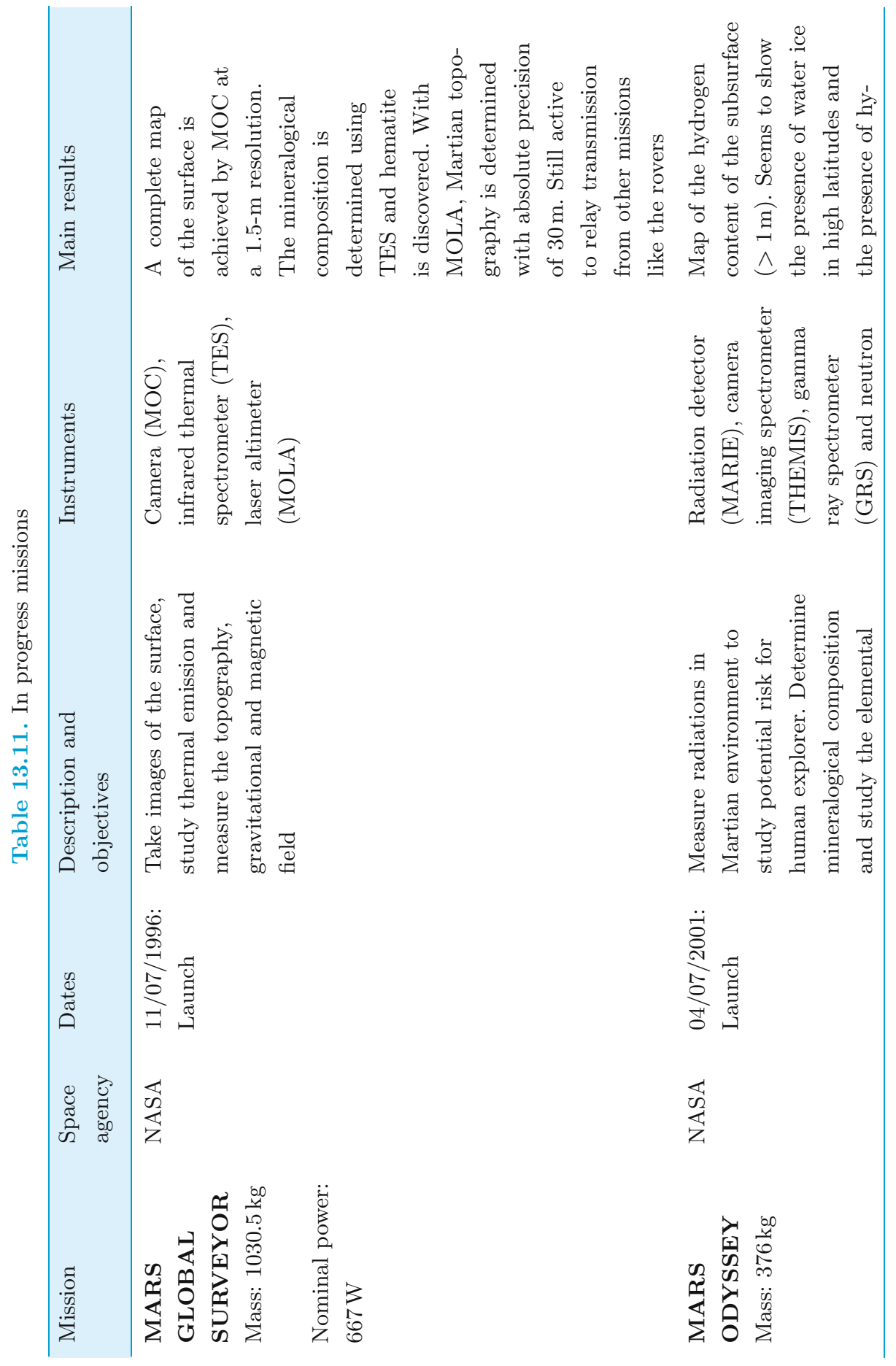




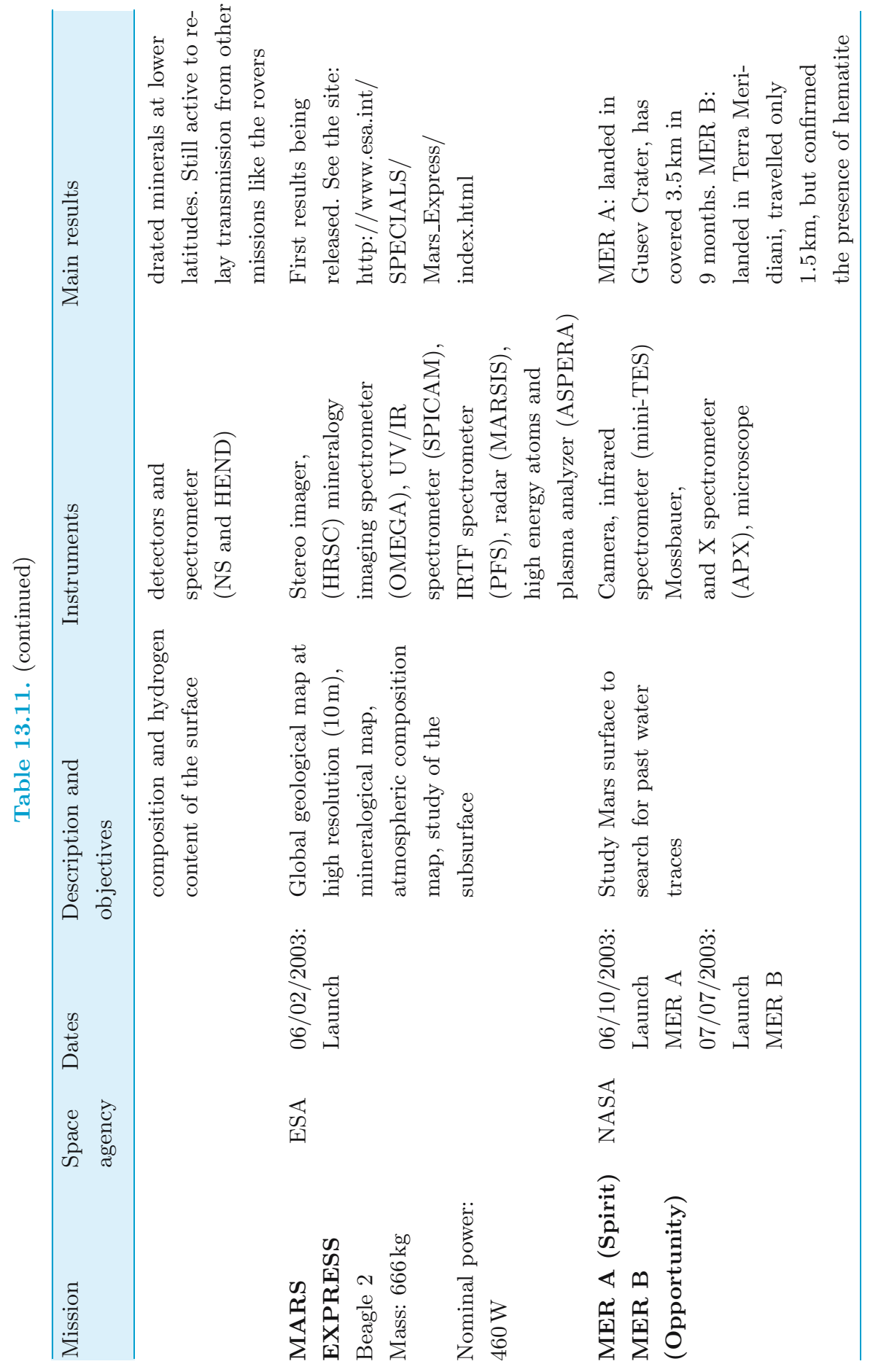




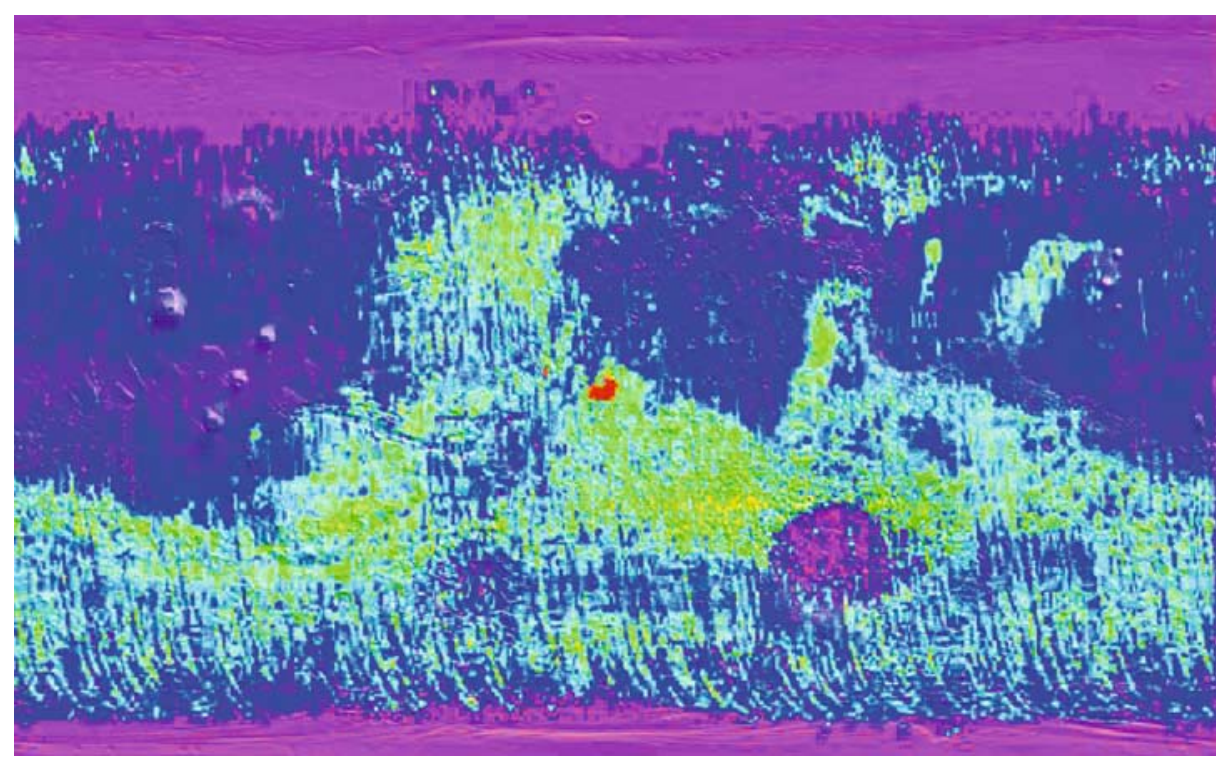

Fig. 13.24. Hematite map on Mars obtained by TES of the MGS mission (Christensen et al. 2000). Regions with high hematite concentration appear in red

the presence of hematite concentrated in small black spherules called "blueberries" (Moore 2004). The rover has also found jarosite, an iron sulfate. It could form if an atmosphere rich in carbon dioxide and sulfur dioxide is in contact with a large water mass rich in iron, (Fairen et al. 2004). Such conditions would preclude the formation of carbonates, which could explain their non-detection until now at the Martian surface. The presence of sulfates in Meridiani Planum has been confirmed by the Omega experiment onboard Mars Express mission (Gendrin et al. 2004). Furthermore, this experiment has allowed the identification of those minerals in and around Valles Marineris. The same instrument also put in light the presence of water ice at the south pole (Bibring et al. 2004).

The mapping of hydrogen present in the ground at less than one meter below the surface, either in the form of ice or in the form of hydrated minerals, has been obtained by the neutron detector and the gamma spectrometer of the Mars Odyssey mission (Fig. 13.25). A large concentration of ice appears at the poles and a low concentration at the equator except in two regions: Arabia Terra and Apollineris. In those regions, the water could be in the form of ice present several tenth of centimeter below a dehydrated layer.

Another instrument onboard Mars Express Mission has made a discovery of large exobiological implications: the infrared Fourier transform spectrometer observed methane (Formisano et al. 2004). This detection has also been confirmed by terrestrial observations (Krasnopolski et al. 2004; Mumma et al. 2004); this 


\section{Water Map \\ 2001 Mars Odyssey Gamma Ray Spectrometer H2O Low H2O High}

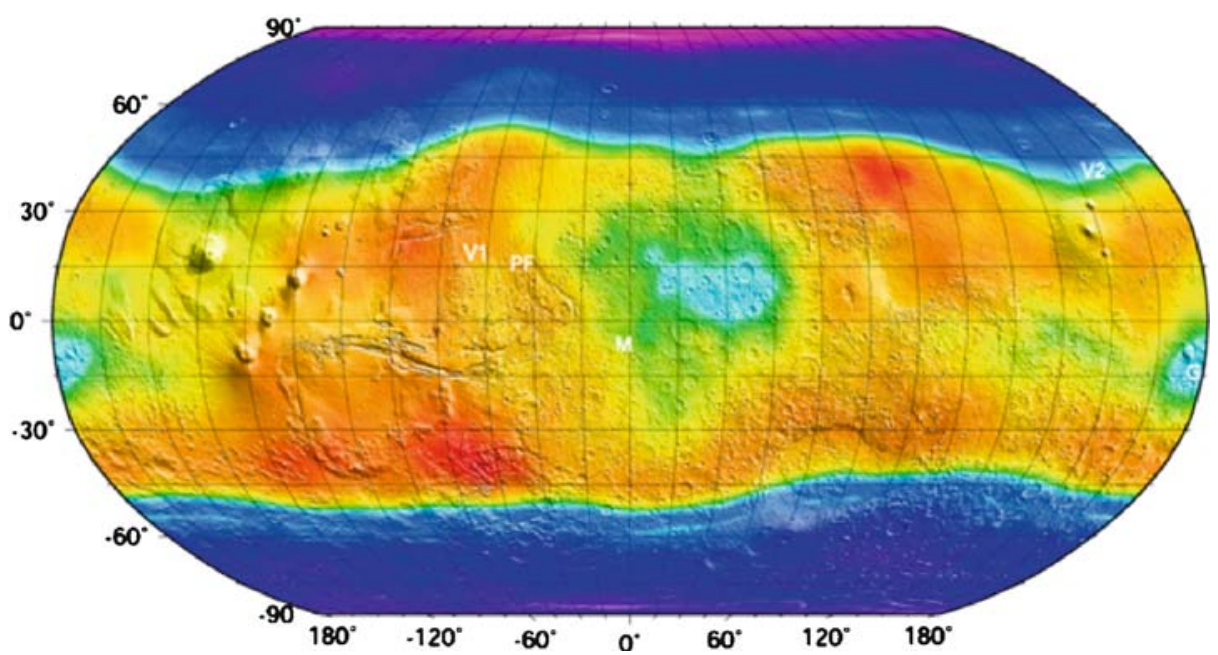

Fig. 13.25. Map created using the hydrogen measurements of the gamma spectrometer of the Mars Odyssey mission (Mitrofanov et al. 2002). If this hydrogen is really in the form of water, regions in blue/violet show the presence of more than $50 \%$ ice by volume in the ground. The significantly drier equatorial regions show two sites more hydrated around Arabia Terra (at center) and Apollineris (on the side). The localizations of the lander missions are indicated: Viking 1 ( VL1), Viking 2 (VL2), Pathfinder $(P F)$, Spirit at Gusev $(G)$, and Opportunity at Meridiani $(M)$

methane has a very low abundance of one part per billion of carbon dioxide. Nevertheless, since the lifetime of methane in Mars atmosphere is of the order of one century, it has to be replenished from a source. On Earth, this source is mainly of biological origin, but Mars volcanism could be the explanation. In the near future, cartography of the methane content in the Martian atmosphere should provide some clues.

\subsubsection{Future Exobiological Missions}

If the results obtained by the exobiological experiment of Viking missions did not allow to definitely conclude on the presence of biological activity on Mars, they had the merit to exist in order to allow the criticism. This helps today to plan mission scenarios optimized for the search of life traces on Mars.

The first action has begun, since as we saw in the previous paragraph, the present program consists of trying to identify the places and the times where liquid water could exist on Mars. A second step will be to search for organic 


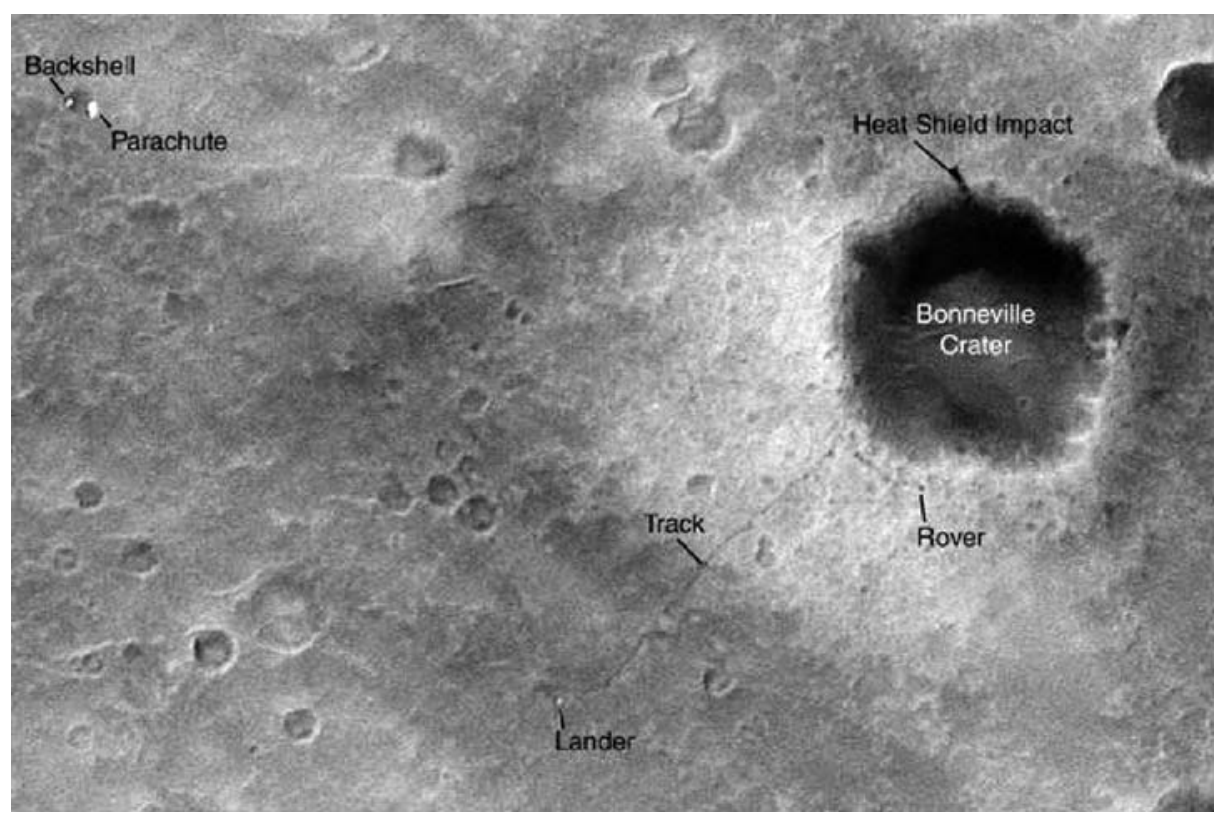

Fig. 13.26. Image acquired on March 30, 2004 by MOC of MGS (resolution $1.5 \times$ $0.5 \mathrm{~m} / \mathrm{pixel}$ ). One can see the landing site of Spirit together with the track of the rover in direction of the Bonneville Crater during 85-sol (image courtesy of NASA/JPL/Malin Space Science Systems)

matter but such a target could be achieved only by in situ measurements. Future missions will also have to go further. They will have to look not only at the Martian surface but also to go deeper inside to explore the subsurface. The reason is that oxidation processes by molecules like hydrogen peroxide $\left(\mathrm{H}_{2} \mathrm{O}_{2}\right)$ recently observed in the atmosphere (Clancy et al. 2004), or superoxides certainly lead to the destruction of organic matter on the surface (Yen et al. 2000). The thickness of the oxidized regolith has been evaluated theoretically but the uncertainties are such that this thickness can vary from one meter to several tenths of a meter (Zent 1998). Consequently, future missions will have to be able to drill at least one meter or even more.

The analytical system will have to be able to extract organic matter from the rock and analyze it to determine its composition. The pyrolysis/GC-MS technique could be improved compared to the one used on the Viking mission. First, derivatization could allow the analysis of refracting molecules that could appear in the degradation of the initial organic material. Then, the use of chiral chromatographic columns could allow the determination of the origin of molecules, either biotic or abiotic (Cabane et al. 2004). Isotopic measurements could also complete such analysis of the organic matter because isotopic ratio can help to identify biotic process. For example, on Earth carbon fractionation $\left({ }^{12} \mathrm{C} /{ }^{13} \mathrm{C}\right)$ in 


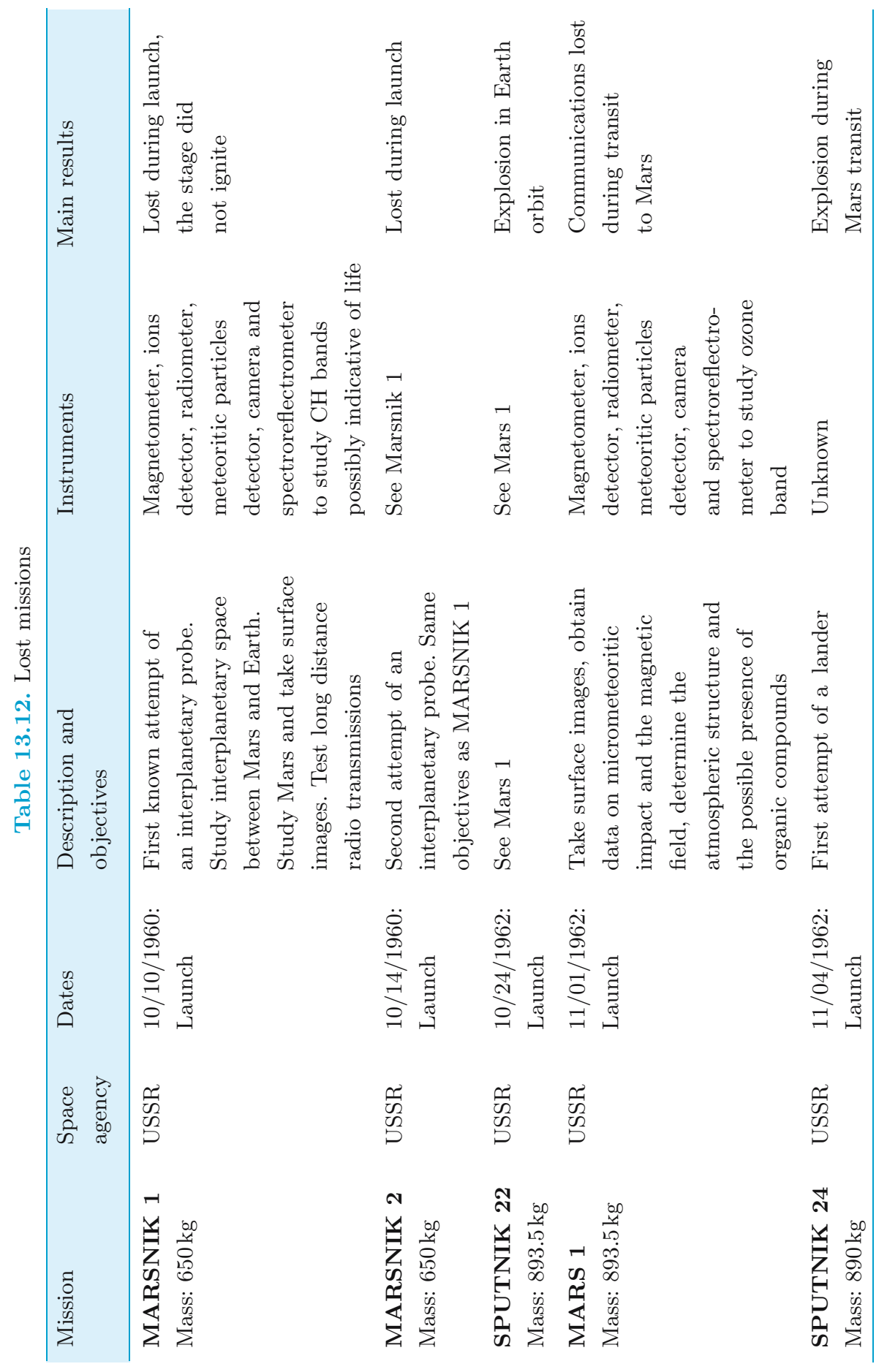




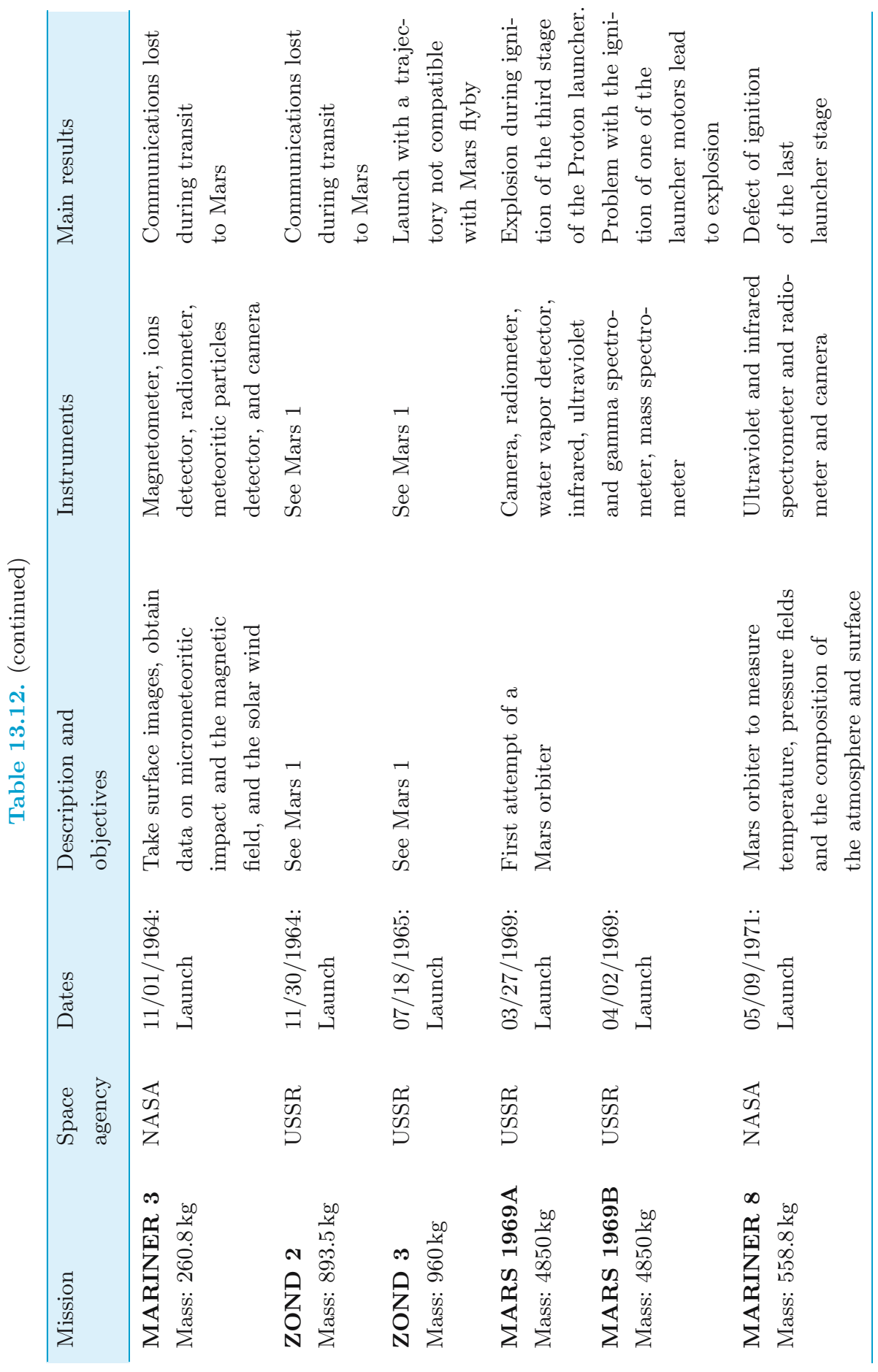




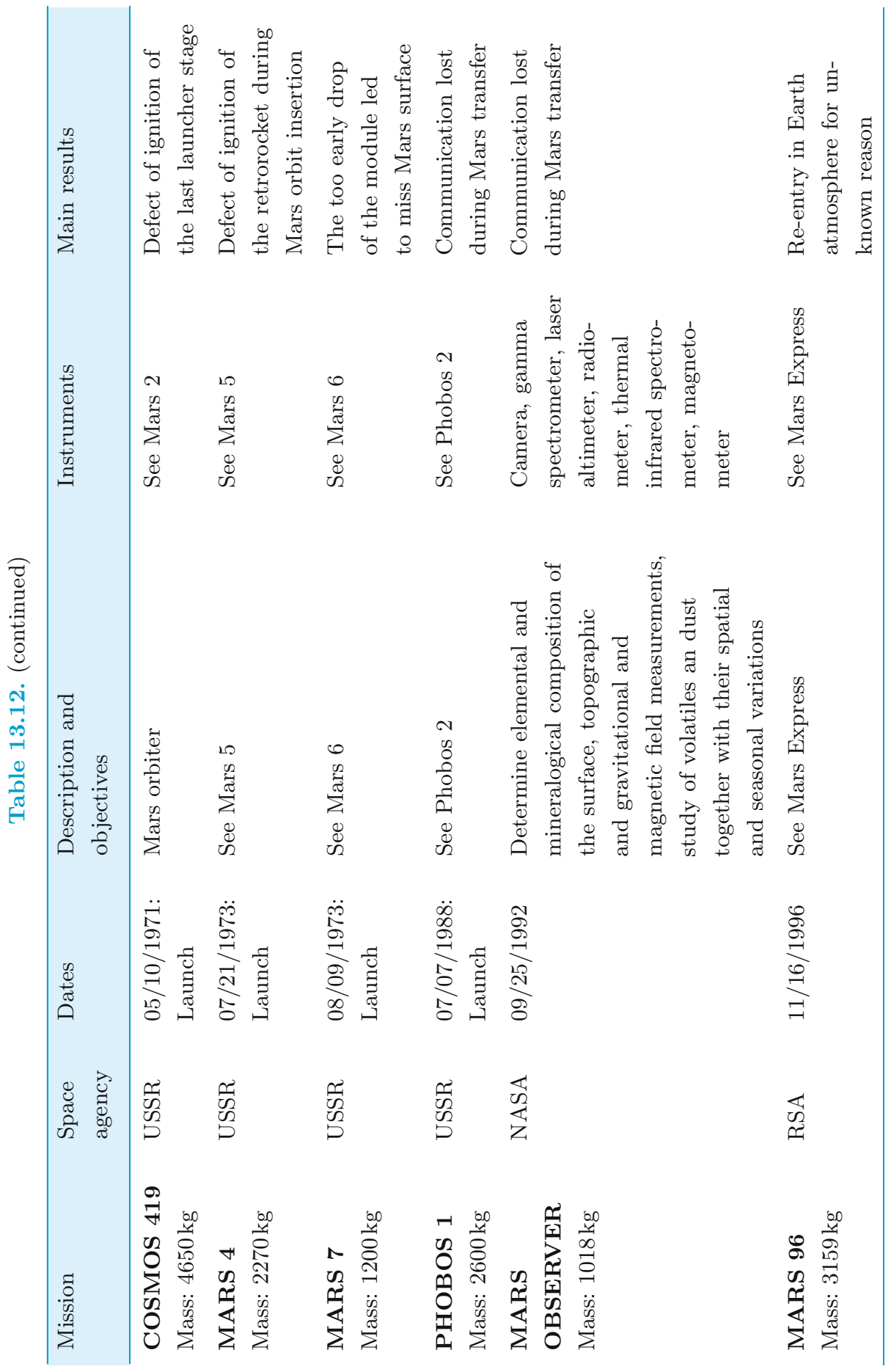




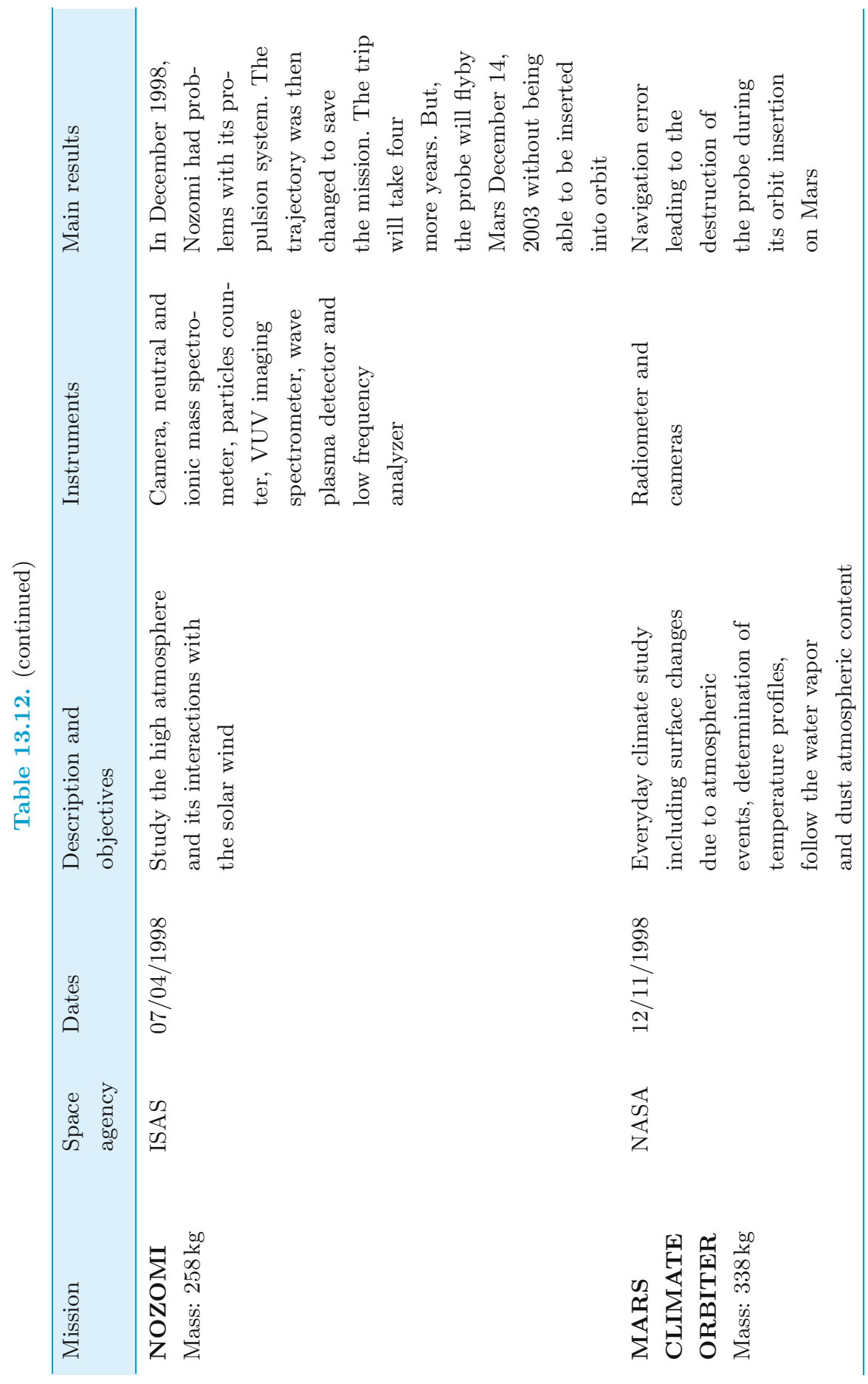




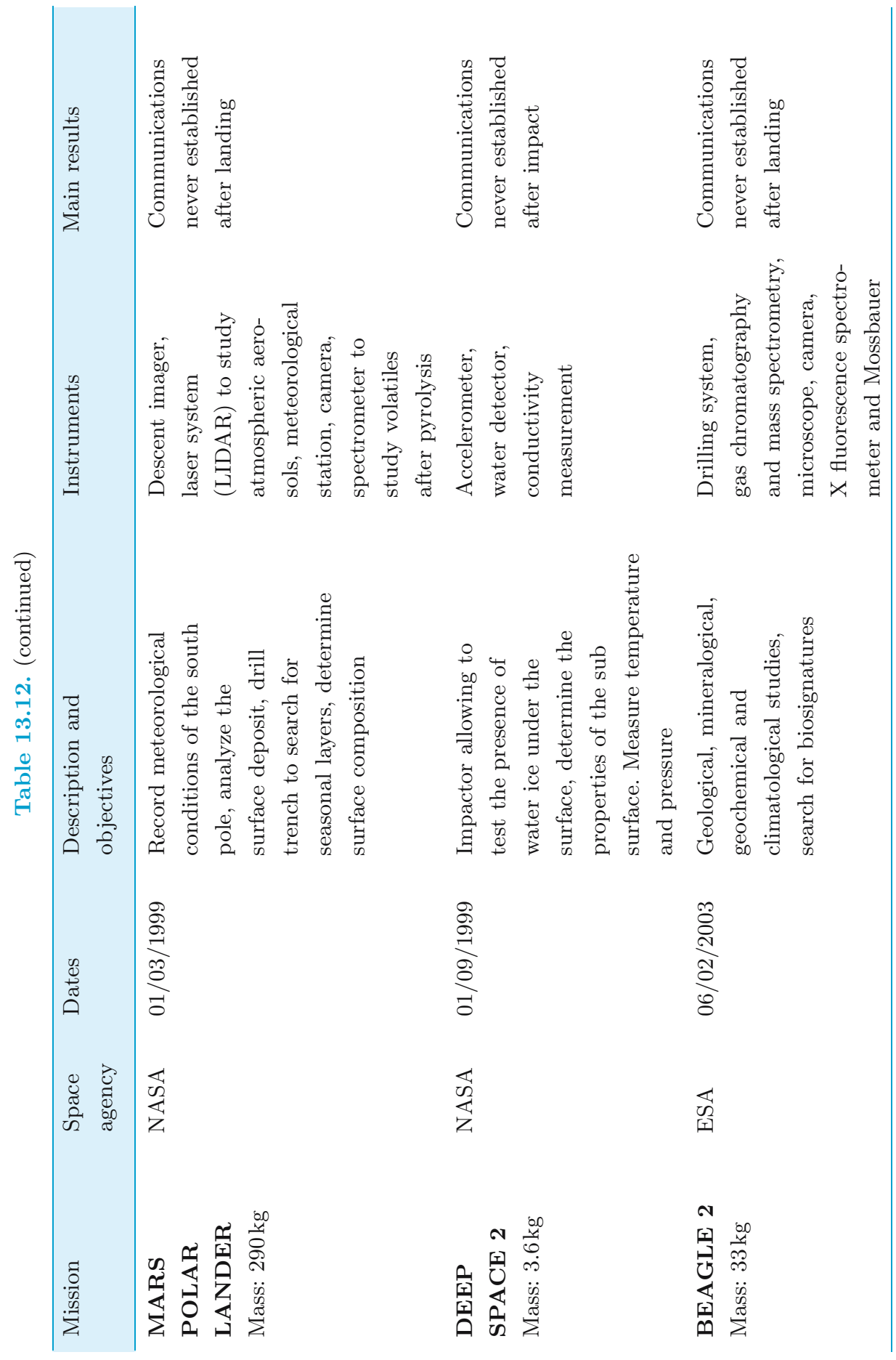




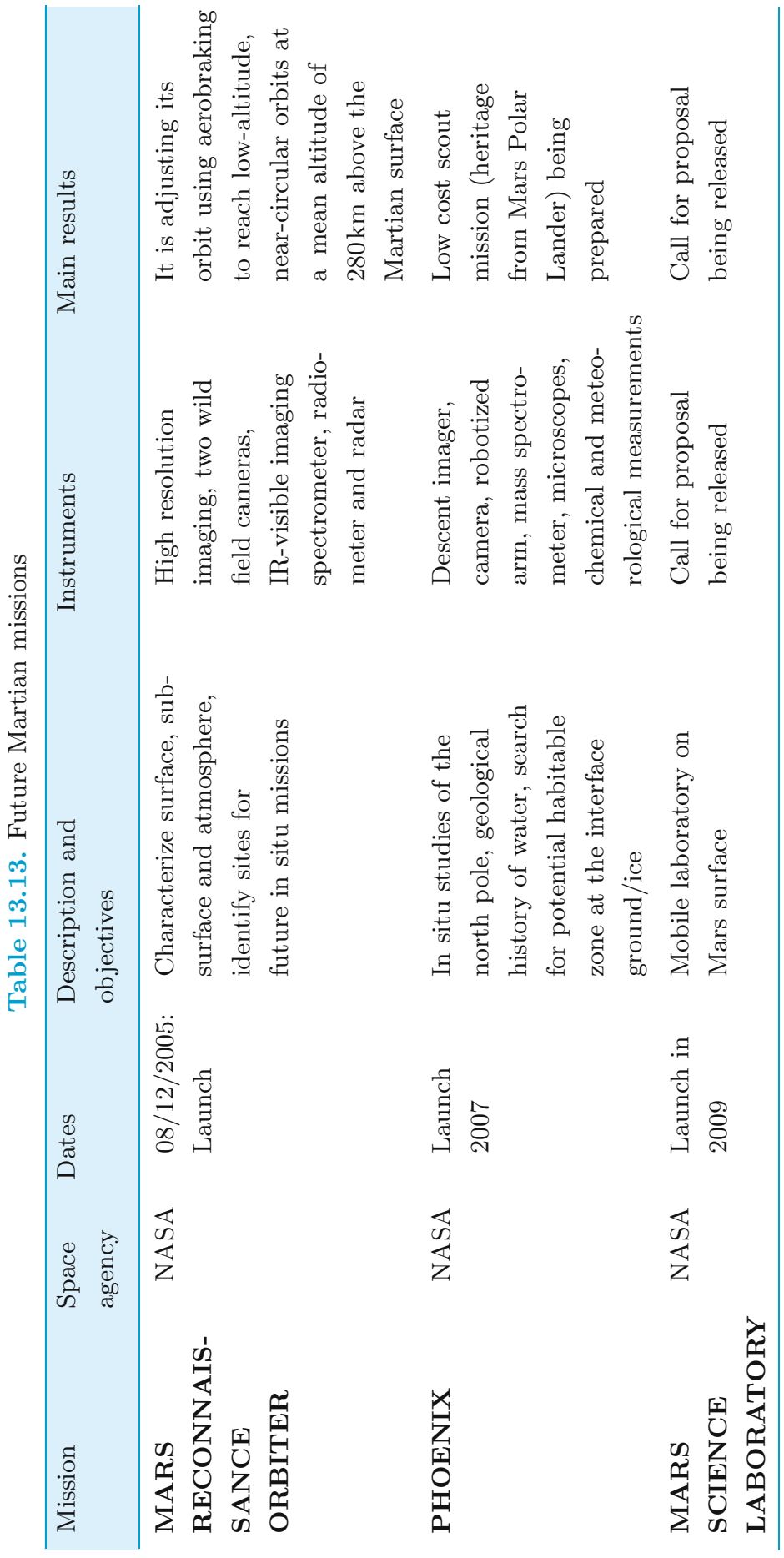


favor of ${ }^{12} \mathrm{C}$ is of the order of 20 to $30 \%$ in the biomass compared to inorganic carbon (Brack et al. 1999). Such measurement set will probably be present on the future MSL mission (Mars Science Laboratory) planned in 2009 (see Table 13.13).

Nevertheless, with all the in situ detection effort of life traces, the sign of possible Martian life could be so subtle that the equipment necessary for its detection would be much too heavy and complex to be put on a space probe. The solution would then be to bring samples collected on Mars back to the Earth to study them in the laboratory. This type of mission initially planned for the end of the decade is actually not expected before 2015 .

\subsection{Conclusion}

To date, there is nothing that could allow us to figure out if we will ever know whether the Earth is the only inhabited planet in the universe. However, astrobiology has gone a long way since the very first experiments by Miller in 1950 and since then, questions related to astrobiology are feeding the exploration of our solar system, and even beyond with ambitious programs for the observation of extrasolar planets (TPF, Darwin). The answer is maybe already at hand, by a rock analyzed with a Martian rover, or further away, under a few kilometers of ice in the Jupiter neighborhood... This chapter deals with the preliminary steps of the astrobiological exploration of the solar system, we are currently witnessing. We focused on the search of organic matter and liquid water, but none of them is a clear indicator of past or present life. We did not discuss the next issue about measurement(s) (chemical, geological, spectroscopic), which could allow us to claim that we have finally detected life on another planet. The scientific debate about it is still at its very first stages, and opens the most fascinating prospects.

\section{Acknowledgements}

The authors wish to thank Antoine Jolly for a careful proofreading of the manuscript; his many comments and suggestions were a great help to improve the text. We also thank the editors of the book: Muriel Gargaud, Philippe Clays and Hervé Martin for their comments, encouragements and corrections. Hervé Martin did a wonderful job in processing the figures.

\section{References}

Altwegg, K., H. Balsiger, and J. Geiss, Composition of the volatile material in Halley's coma from in situ measurements, Space Science Reviews, 90, 3-18, 1999.

Atreya, S.K., T.M. Donahue, and W.R. Kuhn, Evolution of a nitrogen atmosphere on Titan, Science, 201, 611-613, 1978. 
Barbier, B., A. Chabin, D. Chaput, and A. Brack, Photochemical processing of amino acids in Earth orbit, Planetary and Space Science, 46 (4), 391-398, 1998.

Barbier, B., O. Henin, F. Boillot, A. Chabin, D. Chaput, and A. Brack, Exposure of amino acids and derivatives in the Earth orbit, Planetary and Space Science, 50, 353-359, 2002.

Bernstein, M.P., S.A. Sandford, L.J. Allamandola, S. Chang, and M.A. Scharberg, Organic Compounds Produced By Photolysis of Realistic Interstellar and Cometary Ice Analogs Containing Methanol, The Astrophysical Journal, 454, 327-344, 1995.

Bibring, J.P., Y. Langevin, F. Poulet, A. Gendrin, B. Gondet, M. Berthé, A. Soufflot, P. Drossart, M. Combes, G. Bellucci, V. Moroz, N. Mangold, B. Schmitt, and the OMEGA team, Perennial water ice identified in the south polar cap of Mars, Nature, 428, 6983, 627-630, 2004.

Biemann, K., J. Oro, P. Toulmin, L.E. Orgel, A.O. Nier, D.M. Anderson, D. Flory, A.V. Diaz, D.R. Rushneck, and P.G. Simmonds, The search for organic substances and inorganic volatile compounds in the surface of Mars, Journal of Geophysical Research, 82, 4641-4658, 1977.

Bland, P.A., T.B. Smith, A.J.T. Jull, F.J. Berry, A.W.R. Bevan, S. Cloudt, and C.T. Pillinger, The flux of meteorites to the Earth over the last 50000 years, Monthly Notices of the Royal Astronomical Society, 283, 551, 1996.

Blank, J.G., G.H. Miller, M.J. Ahrens, and R.E. Winans, Experimental shock chemistry of aqueous amino acid solutions and the cometary delivery of prebiotic compounds, Origins of Life and Evolution of the Biosphere, 31, 15-51, 2001.

Bockelée-Morvan, D., D. Gautier, F. Hersant, J.-M. Huré, and F. Robert, Turbulent radial mixing in the solar nebula as the source of crystalline silicates in comets, Astronomy and Astrophysics, 384, 1107-1118, 2002.

Bockelée-Morvan, D., J. Crovisier, M.J. Mumma, and H.A. Weaver, The composition of cometary volatiles, In: Comets II, edited by M. Festou, H.U. Keller, and H.A. Weaver, University of Arizona Press, Tucson, AZ, 2004.

Boillot, F., A. Chabin, C. Buré, M. Venet, A. Belsky, M. Bertrand-Urbaniak, A. Delmas, A. Brack, and B. Barbier, The Perseus exobiology mission on MIR: behavior of amino acids and peptides in Earth orbit, Origins of Life and Evolution of the Biosphere, 32, 359-385, 2002.

Botta, O., and J.L. Bada, Extraterrestrial organic compounds in meteorites, Surveys in Geophysics, 23, 411-467, 2002.

Brack, A., La chimie de l'origine de la vie, In: Les traces du vivant, edited by M. Gargaud, D. Despois, J.-P. Parisot, and J. Reisse, pp. 61-81, Presses Universitaires de Bordeaux, Bordeaux, 2003.

Broadfoot, A.L., B.R. Sandel, D.E. Shemansky, J.B. Holberg, G.R. Smith, D.F. Strobel, J.C. McConnell, S. Kumar, D.M. Hunten, S.K. Atreya, T.M. Donahue, H.W. Moos, J.L. Bertaux, J.E. Blamont, R.B. Pomphrey and S. Linick, Science, 212, 206-211, 1981.

Cabane, M., P. Coll, C. Szopa, G. Israël, F. Raulin, R. Sternberg, P. Mahaffy, A. Person, C. Rodier, R. Navarro-Gonzàlez, H. Niemann, D. Harpold, W. Brinckerhoff, Did life exist on Mars? Search for organic and inorganic signatures, one of the goals for "SAM" (sample analysis at Mars), Advances in Space Research, 33, 12, 2240-2245. 2004.

Caldwell, J.J., Ultraviolet observations of small bodies in the solar system by OAO-2, Icarus, 25, 384-396, 1975. 
Carr, M.H., M.J.S. Belton, C.R. Chapman, M.E. Davies, P. Geissler, R. Greenberg, A.S. McEwen, B.R. Tufts, R. Greeley, and R. Sullivan, Evidence for a subsurface ocean on Europa, Nature, 391, 363, 1998.

Christensen, P.R. et al., Detection of crystalline hematite mineralization on Mars by the Thermal Emission Spectrometer, Journal of Geophysical Research, 105, 9632-9642, 2000 .

Christensen, P.R., and S.W. Ruff, Formation of the hematite-bearing unit in Meridiani Planum: Evidence for deposition in standing water. Journal of Geophysical Research, 109, E8, 2004.

Chyba, C.F., P.J. Thomas, L. Brookshaw, and C. Sagan, Cometary delivery of organic molecules to the early earth, Science, 249 (July), 249-373, 1990.

Clancy, R.T., B.J. Sandor, and G.H. Moriarty-Schieven, A measurement of the $362 \mathrm{GHz}$ absorption line of Mars atmospheric H2O2. Icarus, 168, 1, 116-121, 2004.

Conklin, E.K., B.L. Ulich and J.R. Dickel, 3-mm Observations of Titan, Bulletin American Astronomical Society, 9, 471, 1977.

Corliss, J.B., J.A. Baross, and S.E. Hoffman, An hypothesis concerning the relationship between submarine hot spring and the origin of life on Earth, Oceanologica Acta, $\mathrm{N}^{\circ}$ SP, Proceedings of the 26th Geological Congress, 59-69, 1981.

Cottin, H., C. Szopa, and M.H. Moore, Production of hexamethylenetetramine in photolyzed and irradiated interstellar cometary ice analogs, The Astrophysical Journal Letters, 561 (1), L139-L142, 2001.

Cottin, H., Y. Bénilan, M.-C. Gazeau, and F. Raulin, Origin of cometary extended sources from degradation of refractory organics on grains: polyoxymethylene as formaldehyde parent molecule, Icarus, 167, 397-416, 2004.

Coustenis, A., A. Salama, E. Lellouch, T. Encrenaz, G.L. Bjoraker, R.E. Samuelson, T. de Graauw, H. Feuchtgruber, M.F. Kessler, Astronomy and Astrophysics, 336, L85-L89, 1998.

Cronin, J.R., and S. Pizzarello, Enatiomeric excesses in meteoritic amino acids, Science, 275 (14 February), 951-955, 1997.

Curran, R.J., B.J. Conrath, R.A. Hanel, V.G. Kunde, and J.C Pearl, Mars: Mariner 9 spectroscopic evidence for H2O ice clouds, Science 175, 381-383, 1973.

Danielson, R.E., J.J. Caldwell and D.R. Larach, An inversion in the atmosphere of Titan, Icarus, 20, 437-443, 1973.

Despois, D., and H. Cottin, Comets: potential sources of prebiotic molecules for the early Earth, In: Lectures in Astrobiology I, chap. 8, edited by M. Gargaud, B. Barbier, H. Martin, and J. Reisse, Springer, Berlin Heidelberg New York, 2005.

Despois, D., Les comètes, sources potentielles de molécules pour la terre primitive et les planètes, In: L'environnement de la Terre Primitive, edited by M. Gargaud, D. Despois, and J.-P. Parisot, pp. 53-77, Presses Universitaires de Bordeaux, Bordeaux, 2001.

Eberhardt, P., and D. Krankowsky, The electron temperature in the inner coma of comet P/Halley, Astronomy and Astrophysics, 295, 795, 1995.

Ehrenfreund, P., and S.B. Charnley, Organic molecules in the interstellar medium, comets and meteorites: a voyage from dark clouds to the early earth, Annual Review of Astronomy and Astrophysics, 38, 427-483, 2000.

Encrenaz, T., B. Bézard, T.K. Greathouse, M.J. Richter, J.H. Lacy, S.K. Atreya, A S. Wong, S. Lebonnois, F. Lefèvre, and F. Forget. Hydrogen peroxide on Mars: evidence for spatial and temporal variations, Icarus 170, 424-429, 2004. 
Esposito, L.W., et al., The Cassini ultraviolet imaging spectrograph investigation, Space Science Reviews, 115 (1-4), 299-361, 2004.

Fairén, A.G., D. Fernández-Remolar, J.M. Dohm, V.R. Baker, and R. Amils, Inhibition of carbonate synthesis in acidic oceans on early Mars, Nature, 431, 7007, 423-426, 2004.

Flasar, M., et al., Exploring the Saturn system in the thermal infrared: the composite infrared spectrometer, Space Science Reviews, 115 (1-4), 169-297, 2004.

Formisano, V., S. Atreya, T. Encrenaz, N. Ignatiev, and M. Giuranna, Detection of methane in the martian atmosphere, Science, 306, 1756-1761, 2004.

Forterre, P., A la recherche des formes de vie terrestre les plus "primitives": impasses et progrès, In: L'environnement de la Terre Primitive, edited by M. Gargaud, D. Despois, and J.-P. Parisot, pp. 399-416, Presses Universitaires de Bordeaux, Bordeaux, 2001.

Fortes, A.D., Exobiological implications of a possible ammonia-water ocean inside Titan, Icarus, 146, 444-452, 2000.

Fulchignoni, M., F. Angrilli, G. Bianchini, A. Bar-Nun, M.A. Barucci, W. Borucki, M. Coradini, A. Coustenis, F. Ferri, R.J. Grard, M. Hamelin, A.M. Harri, G.W. Leppelmeier, J.J. Lopez-Moreno, J.A.M. McDonnell, C. McKay, F.M. Neubauer, A. Pederson, G. Picardi, V. Pironello, R. Pirjola, R. Rodrigo, C. Sshwingenschuh, A. Seiff, H. Svedhem, E. Thrane, V. Vanzani, G. Visconti and J.C. Zarnecki., Huygens: Science, payload and Mission, ESA SP-1177, 163-195, 1997.

Geiss, J., K. Altwegg, H. Balsiger, and S. Graf, Rare atoms, molecules and radicals in the coma of P/Halley, Space Science Reviews, 90, 253-268, 1999.

Gendrin, A., J.P. Bibring, B. Gondet, Y. Langevin, N. Mangold, J.F. Mustard, F. Poulet, and C. Quantin. Identification of sulfate deposits on Mars by Omega/Mars Express. Proceedings of the 2nd Conference on Early Mars, 11-15 October 2004, Jackson Hole, WY.

Gillett, F.C., Further observations of the 8-13 micron spectrum of Titan, The Astrophysical Journal, 201, L41-L43, 1975.

Gillett, F.C., W.J. Forrest and K.M. Merrill, 8-13 Micron Observations of Titan, The Astrophysical Journal, 184, L93-L95, 1973.

Greenberg, J.M., What are comets made of? A model based on interstellar dust, In: Comets, edited by L.L. Wilkening, pp. 131-163, University of Arizona Press, Tucson, AZ, 1982.

Hanel, R., B. Conrath, F.M. Flasar, V. Kunde, W. Maguire, J. Pearl, J. Pirriglia, R. Samuelson, L. Herath, M. Allison, D. Cruikshank, D. Gautier, P. Gierasch, L. Horn, R. Koppany, and C. Ponnamperuma, Science, 212, 192-200, 1981.

Hanel, R., B. Conrath, W. Hovis, V. Kunde, P. Lowman, W. Maguire, J. Pearl, J. Pirraglia, C. Prabhakara, and B. Schlachman, Investigation of the Martian environment by infrared spectroscopy on Mariner 9, Icarus 17, 423-442, 1972.

Hennet, R.J.C., N.G. Holm, and M.H. Engel, Abiotic synthesis of amino acids under hydrothermal conditions and the origin of life: a perpetual phenomenon, Naturwissenschaften, 79, 361-365, 1992.

Herr, K.C., G.C. Pimental, Infrared absorptions near three microns recorded over the polar CAP of Mars, Science, 166, 496-499, 1969. 
Hersant, F., D. Gautier, and J.-M. Huré, A two-dimensional model for the primordial nebula constrained by $\mathrm{D} / \mathrm{H}$ measurements in the solar system: implications for the formation of giant planets, Astrophysical Journal, 554, 391-407, 2001.

Horowitz, N.H., G.L. Hobby, J.S. Hubbard, Viking on Mars: the carbon assimilation experiments, Journal of Geophysical Research, 82, 4659-4662, 1977.

Huebner, W.F., D.C. Boice, and A. Korth, Halley's polymeric organic molecules, Advances in Space Research, 9 (2), 29-34, 1989.

Huebner, W.F., First polymer in space identified in Comet Halley, Science, 237, 628-630, 1987.

Huguenin, R.L., K.J. Miller, W.S. Harwood, Frost-weathering on Mars: experimental evidence for peroxide formation, Journal of Molecular Evolution, 14, 103-132, 1979.

Hunten, D.M., The Saturn System, edited by D.M. Hunten and D. Morison, NASACP 2068, 113-126, 1978.

Iro, N., D. Gautier, F. Hersant, D. Bockelée-Morvan, and J.I. Lunine, An interpretation of the nitrogen deficiency in comets, Icarus, 161, 511-532, 2003.

Israel, G., H. Niemann, F. Raulin, W. Riedler, S. Atreya, S. Bauer, M. Cabane, E. Chassefière, A. Hauchecorne, T. Owen, C. Sablé, R. Samuelson, J.P. Torre, C. Vidal-Majar, J.F. Brun, D. Coscia, R. Ly, M. Tintignac, M. Steller, C. Gelas, E. Condé, and P. Millan, Huygens: science, payload and mission, ESA SP-1177, 59-84, 1997.

Kasting, J.F., Earth's early atmosphere, Science, 259, 920-926, 1993.

Khare, B.N. and C. Sagan, Red clouds in reducing atmospheres, Icarus, 20, 311, 1973.

Kissel, J., and F.R. Krueger, The organic component in dust from Comet Halley as mesured by the PUMA mass spectrometer on board Vega 1, Nature, 326 (April), 755-760, 1987.

Kissel, J., F.R. Krueger, J. Silén, and B.C. Clark, The cometary and interstellar dust analyzer at comet 81P/Wild 2, Science, 304, 1774-1776, 2004.

Kivelson, M.G., K.K. Khurana, C.T. Russell, M. Volwerk, R.J. Walker, and C. Zimmer, Galileo magnetometer measurements: a stronger case for a subsurface ocean at Europa, Science, 289, 1340-1343, 2000.

Klein, H.P., The Viking biological experiments on Mars, Icarus, 34, 3, 666-674, 1978.

Klein, H.P., J. Lederberg, A. Rich, Biological experiments: the Viking Mars Lander, Icarus, 16, 139, 1972.

Kliore, A., D.L. Cain, G.S. Levy, V.R. Eshleman, G. Fjeldbo, and F.D. Drake, Occultatio experiment: results of the first direct measurement of Mars's atmosphere and ionosphere, Science, 149, 1243-1248, 1965.

Krasnopolsky, V.A., J.-P. Maillard, and T. Owen. Detection of methane in the martian atmosphere: evidence for life? Icarus 172, 537-547, 2004.

Krueger, F.R., A. Korth, and J. Kissel, The organic matter of comet Halley as inferred by joint gas phase and solid phase analyses, Space Science Reviews, 56, 167-175, 1991.

Krueger, F.R., and J. Kissel, The chemical composition of the dust of comet P/Halley as measured by PUMA on board Vega 1, Naturwissenschaften, 74, 312-316, 1987.

Kunde, V.G., A.C. Ainkin, R.A. Hanel, D.E. Jennings, W.C. Maguire, and R.E. Samuelson, Nature, 292, 686-688, 1981.

Lebreton, J.P., and D. Matson, Huygens: science, payload and mission, ESA SP-1177, 1997. 
Levin, G.V., P.A. Straat, A search for a nonbiological explanation of the Viking labeled release life detection experiment. Icarus, 45, 2, 494-516, 1981.

Lindal, G.F., G.E. Wood, H.B. Hotz, D.N. Sweetnam, V.R. Eshleman and G.L. Tyler, Icarus, 53, 348-363, 1983.

Lorenz, R.D., Exo-/astrobiology. Proceedings of the 1st European Workshop, ESA SP-496, 215-218, 2001.

Low, F.J., Planetary radiation at infrared and millimeter wavelengths, Lowell Observatory Bulletin, 6(128), 184-187, 1965.

Lunine, J.I., S. Engel, R. Bashar, and M. Horanyi, Sublimation and reformation of icy grains in the primitive solar nebula, Icarus, 94, 333-344, 1991.

Lutz, B.L., C. de Bergh and T. Owen, Titan: discovery of carbon monoxide in its atmosphere, Science, 220, 1374-1375, 1983.

Maguire, W.C., R.A. Hanel, D.E. Jennings, V.G. Kunde and R.E. Samuelson, Nature, 292, 683-686, 1981.

Marten, A., D. Gautier, L. Tanguy, A. Lecacheux, C. Rosolen and G. Paubert, Icarus, $76,558-562,1988$.

Maurette, M., Carbonaceous micrometeorites and the origin of life, origins of life and evolution of the biosphere, Origins of Life and Evolution of the Biosphere, 28, 385-412, 1998.

Maurette, M., La matière extraterrestre primitive et les mystère de nos origines, In: L'environnement de la Terre Primitive, edited by M. Gargaud, D. Despois, and J.-P. Parisot, pp. 99-127, Presses Universitaires de Bordeaux, Bordeaux, 2001.

McCord, T.B., G.B. Hansen, and C.A. Hibbitts, Hydrated salt minerals on Ganymede's surface: evidence of an ocean below, Science, 292, 1523-1525, 2001.

McCord, T.B., G.B. Hansen, D.L. Matson, T.V. Jonhson, J.K. Crowley, F.P. Fanale, R.W. Carlson, W.D. Smythe, P.D. Martin, C.A. Hibbitts, J.C. Granahan, and A. Ocampo, Hydrated salt minerals on Europa's surface from the Galileo nearinfrared mapping spectrometer (NIMS) investigation, Journal of Geophysical Research, 104, 11827-11852, 1999.

Meier, R., P. Eberhardt, D. Krankowsky, and R.R. Hodges, The extended formaldehyde source in comet P/Halley, Astronomy and Astrophysics, 277, 677-691, 1993.

Meierhenrich, U.J., G.M.M. Caro, J.H. Bredehöft, E.K. Jessberger, and W.H.-P. Thiemann, Identification of diamino acids in the Murchison meteorite, Proceedings of the National Academy of Sciences, 101, 9182-9186, 2004.

Miller, S.L., The production of amino acids under possible primitive Earth conditions, Science, 117, 528-529, 1953.

Mitchell, D.L., R.P. Lin, C.W. Carlson, A. Korth, H. Rème, and D.A. Mendis, The origin of complex organic ions in the coma of comet Halley, Icarus, 98, 125-133, 1992.

Mitrofanov et al., Maps of subsurface hydrogen from the High Energy Neutron Detector, Mars Odyssey, Science, 297, 78-81, 2002.

Möller, G., and W.M. Jackson, Laboratory studies of polyoxymethylene: application to comets, Icarus, 86, 189-197, 1990.

Moore, M.H., and T. Tanabe, Mass spectra of sputtered polyoxymethylene: implications for comets, The Astrophysical Journal, 365, 1990.

Moore, J.M., Mars blueberry fields for ever, Nature, 428, 6984, 711-712, 2004. 
Mumma, M.J., R.E. Novak, M.A. Di Santi, B.P. Bonev, N. Dello Russio, Detection and mapping of methane and water on Mars, Bulletin American Astronomical Society, 36, 1127-1127, 2004.

Muñoz Caro, G.M., and W.A. Schutte, UV-photoprocessing of interstellar ice analogs: new infrared spectroscopic results, Astronomy and Astrophysics, 412, 121-132, 2003.

Ness, F.N., M.H. Acuña, R.P. Lepping, J.E.P. Connerney, K.W. Behannon, L.F. Burlaga and F.M. Neubaueur, Magnetic field studies by Voyager 1: preliminary results at Saturn, Science, 212, 211-216, 1981.

Neugebauer, G., G. Miinch, H. Kieffer, S.C. Chase, and E. Miner, Mariner 1969 infrared radiometer results: temperatures and thermal properties of the Martian surface. The Astronomical Journal, 76, 719, 1971.

Niemann, H. et al., Huygens: science, payload and mission, ESA SP-1177, 85-107, 1997.

Oro, J., and C.B. Cosmovici, Comets and life on the primitive Earth, in astronomical and biochemical origins and the search for life in the universe, edited by C.B. Cosmovici, S. Bowyer, and D. Werthimer, pp. 97-120, Proceedings of the 5th International Conference on Bioastronomy, Bologna, Italy, 1997.

Oyama, V.I., B.J. Berdahl, The Viking gas exchange experiment results from Chryse and Utopia surface samples, Journal of Geophysical Research, vol. 82, 4669-4676, 1977.

Pierazzo, E., and C.F. Chyba, Cometary delivery of biogenic elements to Europa, Icarus, 157, 120-127, 2002.

Pizzarello, S., and J.R. Cronin, Non-racemic amino acids in the Murray and Murchison meteorites, Geochimica et Cosmochimica Acta, 64, 329-338, 2000.

Podolak, M., and D. Prialnik, 26-Al and liquid water environments in comets, In: Comets and the Origin and Evolution of Life, edited by P.J. Thomas, C.F. Chyba, and C.P. McKay, Springer, Berlin Heidelberg New York, 1997.

Pollack, J.B., Greenhouse models of the atmosphere of Titan, Icarus, 19, 43-58, 1973.

Prinn, R.G., and B.J. Fegley, Solar nebula chemistry: origin of planetary, satellite and cometary volatiles, In: Origin and Evolution of Planetary and Satellite Atmospheres, edited by S.K. Atreya, J.B. Pollack, and M. Matthews, pp. 78-136, University Of Arizona Press, Tuscon, AZ, 1989.

Raulin, F., Huygens: science, payload and mission, ESA SP-1177, 219-229, 1997.

Raulin, F., Chimie prébiotique: expériences de simulation en laboratoire et "vérité terrain", In: L'environnement de la Terre Primitive, edited by M. Gargaud, D. Despois, and J.-P. Parisot, pp. 343-360, Presses Universitaires de Bordeaux, Bordeaux, 2001.

Reisse, J., and J. Cronin, Chiralité et origine de l'homochiralité, In: Les traces du vivant, edited by M. Gargaud, D. Despois, J.-P. Parisot, and J. Reisse, pp. 83-113, Presses Universitaires de Bordeaux, Bordeaux, 2003.

Rodier, C., O. Vandenabeele-Trambouze, R. Sternberg, D. Coscia, P. Coll, C. Szopa, F. Raulin, C. Vidal-Madjar, M. Cabane, G. Israel, M.F. Grenier-Loustalot, M. Dobrijevic, and D. Despois, Detection of martian amino acids by chemical derivatization coupled to gas chromatography: in situ and laboratory analysis, Advances in Space Research, 27, 195-199, 2001.

Rosenbauer, H., S.A. Fuselier, A. Ghielmetti, J.M. Greenberg, F. Goesmann, S. Ulamec, G. Israel, S. Livi, J.A. MacDermott, T. Matsuo, C.T. Pillinger, F. Raulin, R. Roll, and W. Thiemann, The Cosac experiment on the lander of the Rosetta mission, Advances in Space Research, 23, 333-340, 1999. 
Samuelson, R.E., W.C. Maguire, R.A. Hanel, V.G. Kunde, D. Jennings, Y.L. Yung and A.C. Aikin, $\mathrm{CO}_{2}$ on Titan, Journal of Geophysical Research, 88, 8709-8715, 1983.

Schenk, P.M., Thickness constraints on the icy shells of the galilean satellites from a comparison of crater shapes, Nature, 417, 419-421, 2002.

Selsis, F., and J.-P. Parisot, L'atmosphère primitive de la Terre et son évolution, In: L'environnement de la Terre Primitive, edited by M. Gargaud, D. Despois, and J.-P. Parisot, pp. 217-233, Presses Universitaires de Bordeaux, Bordeaux, 2001.

Smith, B.A., J. Boyce, G. Briggs, A. Bunker, S.A. Collins, C.J. Hansen, T.V. Johnson, J.L. Mitchell, R.J. Terrile, M. Carr, A.F. Cook II, J. Cuzzi, J.M. Pollack, G.E. Danielson, A. Ingersoll, M.E. Davies, G.E. Hunt, H. Masursky, E. Shoemaker, D. Morrison, T. Owen, C. Sagan, J. Veverka, R. Strom and V.E. Suomi, Encounter with Saturn: Voyager 1 imaging science results, Science, 212, 163-191, 1981.

Smith, G.R., D.F. Strobel, A.L. Bradfoot, B.L. Sandel, D.E. Shemansky and J.B. Holberg, Titan's upper atmosphere: composition and temperature from the EUV solar occultation results, Journal Geophyical Research, 87, 1351-1359, 1982.

Sotin, C., J.W. Head, and G. Tobie, Europa: tidal heating of upwelling thermal plumes and the origin of lenticulae and chaos melting, Geophysical Research Letters, 29, 74-1, 2002.

Stoks, P.G., and A.W. Schwartz, Nitrogen-heterocyclic compounds in meteorites: significance and mechanisms of formation, Geochimica et Cosmochimica Acta, 45, 563-569, 1981.

Stoks, P.G., and A.W. Schwartz, Uracil in carbonaceous meteorites, Nature, 282, 709 $710,1979$.

Stone, E.C. and E.D. Miner, Voyager 1 encounter with the Saturnian system, Science, 212, 159-163, 1981.

Strobel, D.F. and D.E. Shemansky, EUV emission from Titan's upper atmosphere: Voyager 1 encounter, Journal of Geophysical Research, 87, 1361-1368, 1982.

Strobel, D.F., The photochemistry of hydrocarbons in the atmosphere of Titan, Icarus, 21, 466-470, 1974.

Szopa, C., R. Sternberg, F. Raulin, and H. Rosenbauer, What can we expect from the in situ chemical investigation of a cometary nucleus by gas chromatography: first results from laboratory studies, Planetary and Space Science, 51, 863-877, 2003.

Thomas, G.E., Neutral composition of the upper atmosphere of Mars as determined from the Mariner UV spectrometer experiments, Journal of Atmospheric Sciences, 28, 6, 859-868, 1971.

Thompson, W.R., and C. Sagan, Symposium on Titan, ESA SP, 167-176, 1991.

Tomasko, M.G. et al., Huygens: science, payload and mission, ESA SP-1177, 109-138, 1997.

Trafton, L.M., On the possible detection of H_ $\{2\}$ in Titan's atmosphere, The Astrophysical Journal, 175, 285-293, 1972a.

Trafton, L.M., The bulk composition of Titan's atmosphere, The Astrophysical Journal, 175, 295-306, 1972b.

Tyler, G.L., V.R. Eshleman, J.D. Anderson, G.S. Levy, G.S. Lindal, G.E. Wood and T.A. Croft, Radio science investigations of the Saturn system with Voyager 1: preliminary results, Science, 212, 201-206, 1981.

Veverka, J., Titan: polarimetric evidence for an optically thick atmosphere, Icarus, 18, 657-660, 1973. 
Whipple, F.L., A comet model. I. The acceleration of Comet Encke, The Astrophysical Journal, 111, 375-394, 1950.

Wolman, Y., S.L. Miller, J. Ibanez, and J. Oro, Science, 174, 1039, 1971.

Yanagawa, H., and K. Kobayashi, An experimental approach to chemical evolution in submarine hydrothermal systems, Origins of Life and Evolution of the Biosphere, 22, 147-159, 1992.

Yen, A.S., S.S. Kim, M.H. Hecht, M.S. Frant, B. Murray, Evidence that the reactivity of the Martian soil Is due to superoxide ions, Science, 289, 5486, 1909-1912, 2000.

Zarnecki, J.C., M. Banaszkiewicz, M. Bannister, W.V. Boynton, P. Challenor, B. Clark, P.M. Daniell, J. Delderfield, M.A. English, Fulchignoni, M., J.R.C. Garry, J.E. Geake, S.F. Green, B. Hathi, S. Jaroslawski, M.R. Leese, R.D. Lorenz, J.A.M. McDonnell, N. Merrywether-Clarke, C.S. Mill, R.J. Miller, G. Newton, D.J. Parker, P. Rabetts, H. Svedhem, R.F. Turner and M.J. Wright, Huygens: science, payload and mission, ESA SP-1177, 177-195, 1997.

Zellner, B., The polarization of Titan, Icarus, 18, 661-664, 1973.

Zent, A.P., On the thickness of the oxidized layer of the Martian regolith. Journal of Geophysical Research, 103, E13, 31491-31498, 1998.

Zimmer, C., K.K. Khurana, and M.G. Kivelson, Subsurface oceans on Europa and Callisto: constraints from Galileo magnetometer observations, Icarus, 147, 329-347, 2000. 